\title{
Asymptotic Analysis of Perturbations to Travelling Wave Solutions of Nonlinear Advection-Reaction-Diffusion Equations of Fisher Type
}

Wasaif Alruwaele, B. Math (King Faisal University)

\author{
A thesis submitted to \\ the Faculty of Graduate and Postdoctoral Affairs \\ in partial fulfillment of \\ the requirements for the degree of \\ Master of Science
}

School of Mathematics and Statistics

Ottawa-Carleton Institute for Mathematics and Statistics

Carleton University

Ottawa, Ontario, Canada

(C) Copyright

2017, Wasaif Alruwaele 


\section{Contents}

$\begin{array}{lll}\text { Abstract } & \text { ii }\end{array}$

1 Introduction 1

2 Stability of the Equilibrium Solutions 4

2.1 Nondimensionalization . . . . . . . . . . . . . . . . . . . . 8

2.2 Stability of the Equilibrium Solutions for the Case with General $p \quad 10$

2.3 Stability of the Equilibrium Solutions for the Case with General

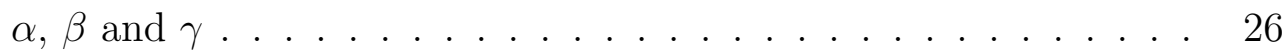

3 Derivation of Travelling Wave Solutions 30

3.1 Introduction . . . . . . . . . . . . . . . . . 30

3.2 Derivation of an Exact Solution . . . . . . . . . . . . . . . 31

3.2.1 Exact solution for the case $\gamma \neq 0 \ldots \ldots \ldots \ldots$

3.2 .2 Exact Solution for the case $\gamma=0 \ldots \ldots \ldots$

4 Perturbations to the Travelling Wave Solutions 42

4.1 Introduction . . . . . . . . . . . . . . . . . . . . 42

4.2 Convergence Theorem . . . . . . . . . . . . . . . . . . 43

4.3 Perturbation to the Initial Condition $\ldots \ldots \ldots$

$4.3 .1 \quad \xi$-dependent Perturbation . . . . . . . . . . . . 48

4.3.2 $\xi$-, $t$ - and $x$-dependent Perturbation . . . . . . . 53 
4.4 Perturbation to the Velocity . . . . . . . . . . . . . . 59

$\begin{array}{lll}5 & \text { Conclusions } & 67\end{array}$

A Definitions and Theorems for the stability of systems of ODEs 70

B The WKB Approximation $\quad 74$

$\begin{array}{lc}\text { Bibliography } & 6\end{array}$ 


\begin{abstract}
In this thesis we examine some nonlinear advection-diffusion-reaction equations of Fisher type. First we discuss the stability properties of the equations by writing each nonlinear equation as a system of two ordinary different equations and then analyzing the stability of their equilibrium solutions and plotting their trajectories in phase portraits. We then describe the derivation of some exact expressions for travelling wave solutions which had been obtained by previous researcher using other methods.

We examine some situations where the exact travelling wave solutions are perturbed. First we perturb the initial condition and then derive approximate expressions for the perturbations. The goal of the thesis is to investigate the case where the constant speed of the background medium is perturbed by a smallamplitude spatially and temporally localized perturbation.

This situation occurs in atmospheric fluid flows when atmospheric waves interact and cause localized perturbations in the background flow speed. The perturbations in the flow speed affect the propagation of chemical species in the atmosphere. The approximate asymptotic solutions derived here show that the form of the perturbation can be exponentially decaying or oscillatory and this depends on the spatial and temporal width of the background speed perturbation.
\end{abstract}




\section{Acknowledgements}

Throughout the course of my Masters degree at Carleton University, I have had to meet and overcome several challenges which would have been much more stressful had I not had the support of several individuals, which has made a huge impact on the experience that I have had here. First, I would like to thank my supervisor, Lucy Campbell, who has been an amazingly supportive person since I started working on my Masters in 2014. Professor Campbell has been there for me when I needed advice regarding anything, even non academic issues. She has always had the answers to all of my questions and words of motivation during times of stress. I would also like to thank my father and mother who have always supported and encouraged me in all my goals; they pushed me to come to Canada to study and develop and I would not be the person I am today without their guidance. I must also give thanks to my husband, Suliman, who has also supported me during my studies,and my life. Without him I would not have had the time to have completed and accomplished all that I have done at Carleton University. Of course I would also like to thank my friends who have been there for me during my time in Canada. I would also like to thank the government of the Kingdom of Saudi Arabia for the opportunity to study overseas. I know that these scholarships and the money to fund them are not available to everyone and that I should feel very thankful for the experience that I have had in Canada. I have been able to expand my cultural horizons, my proficiency in English, and my knowledge in applied mathematics. This study abroad program is excellent for the development of Saudi youth, the education system of the country, and the future of the society. I hope that I can have the opportunity upon returning to Saudi Arabia to be able to spread the knowledge that I gained in Canada to Saudi youth who did not get the same chance in life that I did. 


\section{Dedication}

I would like to dedicate my thesis to my mother and father, my sisters and

brothers, and my husband and daughters (Jawaher, Rhahf and Ghina). They have always been my inspiration in my life and my motivation to achieve all that I have achieved. Without their support and love I would not be where I am today. I hope that someday my daughters will grow up and read this and realize the work and time that I spent during the most precious years of their lives in order to help bring prosperity to my family and our society. 


\section{Chapter 1}

\section{Introduction}

This thesis examines nonlinear advection-diffusion-reaction equations of form

$$
\frac{\partial u}{\partial t}+\bar{c} \frac{\partial u}{\partial x}=\nu \frac{\partial^{2} u}{\partial x^{2}}+F(u)
$$

where $\bar{c}, \nu$ are real constants, $u$ is a real-valued function of the variables $x$ and $t$ and $F$ is a nonlinear function of one of the forms

$$
F(u)=u\left(\gamma-\beta u^{p}\right)
$$

or

$$
F(u)=u\left(\gamma-\beta u-\alpha u^{2}\right)
$$

where $\alpha, \beta, \gamma$ and $p$ are positive constants and $p>0$. Variants of this equation are used to model different physical phenomena including chemical reactions in the atmosphere (Stockie, 2011) and other fluids and biological population dynamics (Fisher, 1937). When $\bar{c}=0$, with $\gamma=1, \beta=1$ and $p=1$ in (1.2) (or $\gamma=1$, $\beta=1$ and $\alpha=0$ in (1.3)), then equation (1.1) becomes

$$
\frac{\partial u}{\partial t}+\bar{c} \frac{\partial u}{\partial x}=\nu \frac{\partial^{2} u}{\partial x^{2}}+u(1-u)
$$

which is known as the Fisher equation.

In general, the variable $t$ denotes time, $x$ denotes one-dimensional space and $u(x, t)$ is some quantity that varies in space and time such as the concentration 
of a chemical species within some fluid environment or the number of members of a population in molecular or cellular biology (Canosa, 1973). In mathematical terms, $u$ could be any complex-valued function but in many physical situations of interest $u$ is intended to represent a physical quantity that is real and takes non-negative values only on a domain given by $-\infty<x<\infty, 0 \leq t<\infty$. In theses situations the solutions that are considered are those that satisfy these characteristics in this domain.

The quantity $\nu$ is the diffusion coefficient which represents the rate of diffusion of the species or other medium under consideration and it is positive in all physically realistic situations and $\bar{c}$ represents the speed of advection or movement of the medium with time in a given domain. In general, $\bar{c}$ and $\nu$ could be functions of $x$ and $t$, but in this thesis we consider special cases where $\nu$ is constant and where $\bar{c}$ is either constant or takes the form of a constant plus a small perturbation that depends on $x$ and $t$. The case where $\nu=0$ and $F=0$ in (1.1) gives the advection equation, which also called the first-order wave equation,

$$
\frac{\partial u}{\partial t}+\bar{c} \frac{\partial u}{\partial x}=0 .
$$

When $\bar{c}$ is constant, the solutions of this equation are functions of the variable $(x-\bar{c} t)$.

The case where $\bar{c}=0$ in (1.1) gives the reaction-diffusion equation

$$
\frac{\partial u}{\partial t}=\nu \frac{\partial^{2} u}{\partial x^{2}}+F(u) .
$$

Even in the absence of the advection term, equation (1.5) admits travelling wave solutions that depend on the variable $\xi=x-c t$, where $c$ is a constant speed. By definition, a travelling wave is a wave that travels with time in such a way that the shape of the solution is the same for all time and the speed of propagation of this shape is constant (Murray, 1989). Equation (1.5) is generally studied rather than (1.1) because the solutions of (1.1) are of the same form as those of (1.5) but with the value of $c$ changed by an increment of $\bar{c}$. If $c>0(c<0)$ the 
wave travels in the direction of positive (negative) $x$ as $t$ increases. Since the equation is invariant if $x$ is replaced by $-x$ and $c$ replaced by $-c$, it is sufficient to consider the case where $c>0$. Rewriting equation (1.2) in terms of the wave variable $\xi=x-c t$ leads to a second-order ordinary differential equation (ODE) for $U(\xi)=u(x, t)$, which can then written as a system of two first order ODEs. A straightforward linear stability analysis, summarized in chapter 2, shows that there is a critical point corresponding to the zero equilibrium solution, as well as one saddle point (if $p$ is odd) or two saddle points (if $p$ is even). If $c \geq 2 \sqrt{\gamma}$ then the zero critical point is a stable node. In this case, there are travelling wave solutions for which $U>0$ for all $\xi$. If $c<2 \sqrt{\gamma}$, the zero critical point is a stable spiral and the solutions are oscillatory functions of $\xi$. In this thesis, we shall focus on the case where $c>2 \sqrt{\gamma}$ and there are travelling wave solutions.

For certain values of $c>2 \sqrt{\gamma}$, it is possible to find an exact solution of (1.5) in closed form. Ablowitz and Zeppetella (1979) considered the case of the Fisher equation (1.4) with $\nu=1$ and showed that, for $c=\frac{5}{\sqrt{6}}$, the solution can be written in the form

$$
u(\xi)=f(\xi) w(s)+g(\xi), s=h(\xi),
$$

where $\xi=x-c t, g(\xi)=0, f(\xi)=\exp (\lambda \xi), h(\xi)=\frac{1}{\sqrt{6}} \exp (-(c+2 \lambda) \xi)$ and $\lambda$ is constant. This leads to the ODE

$$
w^{\prime \prime}=6 w^{2}
$$

which has solution $w(s)=\wp\left(s-k ; O, g_{3}\right)$ where $k$ and $g_{3}$ are arbitrary constants and $\wp$ is the Weierstrass $\wp$-function. This is the elliptic function whose properties are given in section 18.1 of Abramowitz and Stegun (1964).

Otwinowski et al. (1988) considered the general equation of the form

$$
\frac{\partial u}{\partial t}=\frac{\partial^{2} u}{\partial x^{2}}-P(u)
$$

where $P$ is an $n$-th degree polynomial with $n>1$. They found that for certain forms of $P$, a travelling wave solution could be constructed by defining $\xi=x-c t$, 
$U(\xi)=u(x, t)$ where $\frac{d U}{d \xi}=R(u)$ and $R$ is polynomial. A special case of (1.6) is the Fisher equation for which $P(u)=u-u^{2}$. Feng (2007) considered the case of equation (1.1) with (1.3) where $\beta$ and $\gamma$ are both allowed to be non-zero. After making the change of variables $\xi=x-c t$ and $U(\xi)=u(x, t)$, he introduced new variables $\tau$ and $q$ given by

$$
\xi=-\frac{1}{c} \ln \tau, \quad q=\tau^{m}
$$

This transforms equation (1.1) into a form which leads to a travelling wave solution under certain conditions and he expressed the solution in terms of elliptic functions. Yuan et al. (2013) derived a solution in closed form for the Fisher equation((1.4) with $\bar{c}=0, \nu=1)$. Szozda (2014) observed that for the case of the Fisher equation (1.4) the transformation of Feng (2007) could be used to obtain the solution of Ablowitz and Zeppetella (1979) expressed in terms of the Weierstrass elliptic function. A special case of this leads to an exact solution written in terms of exponential functions which is the same as the solution that was obtained by Yuan et al. (2013) using a different method. We see in this thesis that the Feng (2007) transformation can be also applied to the more general equation of the form (1.1) with (1.2) and an exact solution can be obtained under certain conditions. The exact solutions $u(x, t)=U(\xi)$ we obtain for (1.1) satisfy the condition that $U \rightarrow \sqrt{\frac{\gamma}{\beta}}$ as $\xi \rightarrow-\infty$ and $U \rightarrow 0$ as $\xi \rightarrow \infty$. These solutions correspond to some specific initial conditions $u(x, 0)=f(x)$ where $f$ satisfies $f \rightarrow \sqrt{\frac{\gamma}{\beta}}$ as $x \rightarrow-\infty, f \rightarrow 0$ as $x \rightarrow \infty$.

With other more general initial conditions, while it may not be possible to obtain an exact solution in closed form, it would be desirable to obtain information about the qualitative behaviour of the solution for finite time and for infinite time. Kolmogorov et al. (1937) proved a theorem concerning the convergence of solutions of the Fisher equation (1.4). The theorem states that for the Fisher 
equation, every initial condition of the form

$$
u(x, 0)=f(x)>0,
$$

with

$$
f(x)= \begin{cases}1 & \text { if } x<x_{1} \\ 0 & \text { if } x>x_{2}\end{cases}
$$

where $x_{1}<x_{2}$ and $f(x)$ is continuous for $x_{1}<x<x_{2}$, leads to a travelling wave solution of the form $u(x, t)=U(x-c t)$ with $c<2$. Many studies have focused on generalizing the Kolmogorov theorem, e.g. Fife and McLeod (1975, 1977, 1981), Uchiyama (1978), Bramson (1983) and Bramson (1986).

Fife and McLeod (1977) proved that for the nonlinear reaction-diffusion equation (1.5) with $\nu=1$ defined for $-\infty<x<\infty, t>0$, with initial condition $u(x, 0)=f(x),-\infty<x<\infty$, has unique bounded classical solution $u(x, t)$ under certain conditions on the functions $F(u)$ and $f(x)$. Kolmogorov's theorem was generalized by Bramson (1983) to give necessary and sufficient conditions for arbitrary initial conditions to converge to travelling wave solutions.

Given an initial condition of the form (1.7) we consider a situation where the initial function $f$ can be written as

$$
f(x)=f^{(0)}(x)+\varepsilon f^{(1)}(x)
$$

where $f^{(0)}(x)$ is a specified initial function that leads to a known travelling wave solution in closed form $u^{(0)}(x, t)=f^{(0)}(x-c t)$, and $f^{(1)}(x)$ is a function that satisfies $f^{(1)} \rightarrow 0$ as $x \rightarrow \pm \infty$ and $\varepsilon$ is a positive constant. If $\varepsilon \ll 1$, then the solution of (1.1) subject to the initial condition

$$
u(x, 0)=f(x)=f^{(0)}(x)+\varepsilon f^{(1)}(x),
$$

can be expressed as a perturbation series

$$
u(x, t)=u^{(0)}(x, t)+\varepsilon u^{(1)}(x, t)+O\left(\varepsilon^{2}\right), \quad \varepsilon \rightarrow 0 .
$$


The function $u^{(1)}(x, t)$ is the perturbation to the travelling wave solution $u^{(0)}(x, t)=$ $f^{(0)}(x-c t)$ that arises as a result of perturbing the initial function $f^{(0)}(x)$.

Murray (1989) presented a stability analysis of a perturbation to the travelling wave solution. In this thesis we investigate different mechanisms that lead to a perturbation to the travelling wave solution. First, we examine the behaviour of a perturbation $u^{(1)}$ that arises from perturbing the initial condition as in (1.10). Then we consider a situation where the constant speed $\bar{c}$ in (1.1) is modified by the addition of a small perturbation that depends on $x$ and $t$ in some localized region in space and time, i.e., we write $\bar{c}$ as

$$
\bar{c}(x, t)=\bar{c}^{(0)}+\varepsilon \bar{c}^{(1)}(x, t),
$$

where $\varepsilon \ll 1, \bar{c}^{(0)}$ is constant and $\bar{c}^{(1)}$ satisfies $\bar{c}^{(1)} \rightarrow 0$ as $x \rightarrow \pm \infty, \bar{c}^{(1)} \rightarrow 0$ as $t \rightarrow \infty$. We apply an initial condition $u(x, 0)=f(x)$ which would lead to a travelling wave solution in closed form if $\bar{c}$ was constant. If $\bar{c}$ is perturbed as in (1.11), then the solution of (1.1) takes the form

$$
u(x, t)=u^{(0)}(x, t)+\varepsilon u^{(1)}(x, t)+O\left(\varepsilon^{2}\right),
$$

where $u^{(0)}(x, t)=f(x-c t)$ and $c$ depends on $\bar{c}$. The function $u^{(1)}(x, t)$ is the perturbation to the travelling wave solution $u^{(0)}(x, t)$ and it arises as a result of perturbing the constant speed of advection of the medium.

This problem in which the speed of advection of the medium is perturbed arises in certain physical contexts. For example, in the situation where $u$ represents the concentration (or number density) of certain chemical species in the atmosphere and $\bar{c}$ represents the speed of the background wind, interactions between atmospheric waves can give rise to spatially and temporally localized perturbations to the background wind speed $\bar{c}$. The perturbations in the wind speed in turn affect the propagation of the chemical species in the atmosphere. In practice, the concentration of the chemical species also affects the background wind and oscillations and these effects can be represented by means of fluid dynamics 
equations which are coupled to the chemical reaction-diffusion equations. In this thesis we consider a simple model where the background wind $\bar{c}$ is perturbed by a specified function and we examine the response of the chemical species given by $u$ but we do not examine the effects of the chemical species on the background wind.

An overview of the thesis is as follows. Chapters 2 and 3 review well-known properties of nonlinear reaction-diffusion equations of Fisher-type. In chapter 2 we review the stability properties of the equilibration solutions of the nonlinear systems of ordinary differential equations associated (1.1) with (1.2) and (1.3).

In chapter 3 we use the transformation of Feng (2007) to derive travelling wave solutions for (1.1) with (1.2), generalizing the analysis of Szozda (2014) with $\gamma=1, \beta=1$ and $p=1$ or $p=2$ to the situation with general $\gamma, \beta$ and $p$. The solutions obtained are well-known in the literature (e.g. Ablowitz and Zeppetella (1979), Yuan et al. (2013)).

In chapter 4 we review the convergence theorem of Bramson and then derive asymptotic solutions for perturbations to the travelling wave solutions obtained in chapter 3 . In section 4.2 we consider a perturbation to the initial function of the form (1.10) and in section 4.3 we consider a perturbation of the form (1.11) to the advection speed of the background medium.

In chapter 5 we summarize our conclusions. In Appendix A we collect some definitions and theorems concerning the stability of systems of ordinary deferential equations. In Appendix B we provide some information about the WKB method. 


\section{Chapter 2}

\section{Stability of the Equilibrium Solutions}

\subsection{Nondimensionalization}

In this chapter we consider solutions of the form $u(x, t)=U(x-c t$ ) (where $c$ is constant) for the reaction diffusion equation (1.5) with (1.2) and (1.3). The change of independent variable $\xi=x-c t$ leads to a system of two nonlinear ordinary differential equations (O.D.Es). We discuss the stability of the equilibrium solutions of the nonlinear system and conditions that allow travelling wave solutions.

Throughout the thesis, we will deal with variants of equation (1.1) or (1.5) written in terms of nondimensional variables and parameters. In terms of dimensional quantities (with asterisks), the advection-diffusion-reaction equation (1.1) with (1.3) is

$$
\frac{\partial u^{*}}{\partial t^{*}}+\tilde{c}^{*} \frac{\partial u^{*}}{\partial x^{*}}=\nu^{*} \frac{\partial^{2} u^{*}}{\partial x^{* 2}}+u^{*}\left(\gamma^{*}-\beta^{*} u^{*}-\alpha^{*} u^{* 2}\right),
$$

where $\nu^{*}$ is the diffusion coefficient and $\tilde{c}^{*}$ is the speed of the background medium, both are considered to be real constants and $\nu^{*}>0$. In addition, $\gamma^{*}, \beta^{*}$ and $\alpha^{*}$ are 
real parameters and are assumed to be positive. We introduce the nondimensional quantities

$$
x=\frac{x^{*}}{X}, \quad t=\frac{t^{*}}{T}, \quad u=\frac{u^{*}}{U}, \quad \gamma=\frac{\gamma^{*}}{D}, \quad \beta=\frac{\beta^{*}}{B}, \quad \alpha=\frac{\alpha^{*}}{A}, \quad \nu=\frac{\nu^{*}}{V}, \quad \bar{c}=\frac{\tilde{c^{*}}}{C},
$$

where $X$ and $T$ are reference values of the spatial and temporal variables, $U$ is a reference value of the dependent variable $u^{*}$ and $D, B, A, V$ and $C$ are reference values of the parameters $\gamma^{*}, \beta^{*}, \alpha^{*}, \nu^{*}$ and $\tilde{c}^{*}$, respectively.

Substituting (2.2) into (2.1) and multiplying each term by $\frac{T}{U}$ gives

$$
\frac{\partial u}{\partial t}+\frac{T C}{X} \bar{c} \frac{\partial u}{\partial x}=\frac{T V}{X^{2}} \nu \frac{\partial^{2} u}{\partial x^{2}}+u\left(T D \gamma-T B U \beta u-T A U^{2} \alpha u^{2}\right) .
$$

We consider the situation where the diffusion coefficient in the nondimensional equation is an $O(1)$ parameter. In order to obtain this, we scale the diffusion coefficient by using a reference value of $V=\nu^{*}$ so that the nondimensional coefficient $\nu=\frac{\nu^{*}}{V}=1$ and we choose a reference length $X=\left(T \nu^{*}\right)^{\frac{1}{2}}$ so that $\frac{T \nu^{*}}{X^{2}}=1$ and choose $D, B, A$ and $C$ so that

$$
T D=1, \quad T B U=1, \quad T A U^{2}=1, \quad \frac{T C}{X}=1 .
$$

In terms of $T, U$ and $\nu^{*}$, we then have nondimensional quantities

$$
x=\frac{x^{*}}{\sqrt{T \nu^{*}}}, \quad \gamma=T \gamma^{*}, \quad \beta=T \beta^{*} U, \quad \alpha=T \alpha^{*} U^{2}, \quad \bar{c}=\frac{T \tilde{c}^{* \frac{1}{2}}}{\nu^{* \frac{1}{2}}},
$$

and equation (2.1) can then be written in nondimensional form as

$$
\frac{\partial u}{\partial t}+\bar{c} \frac{\partial u}{\partial x}=\frac{\partial^{2} u}{\partial x^{2}}+u\left(\gamma-\beta u-\alpha u^{2}\right)
$$

The same choice of reference quantities applied to equation (1.1) with (1.2) gives the nondimensional equation

$$
\frac{\partial u}{\partial t}+\bar{c} \frac{\partial u}{\partial x}=\frac{\partial^{2} u}{\partial x^{2}}+u\left(\gamma-\beta u^{p}\right)
$$




\subsection{Stability of the Equilibrium Solutions for the Case with General $p$}

In sections 2.2 and 2.3, we summarize the stability properties of the reaction diffusion equation (1.5) with the nonlinear term (1.2) or (1.3) and present some different special cases. These stability properties are well known and can be found for example in the book by Murray (1989).

We consider the nondimensional equation (2.7) with $\bar{c}=0$ and seek a solution of the form

$$
u(x, t)=U(x-c t)=U(\xi), \quad \xi=x-c t .
$$

This is a travelling wave solution with wave speed $c$. Substituting (2.8) into (2.7) gives the ordinary differential equation (O.D.E.)

$$
U_{\xi \xi}+c U_{\xi}+\gamma U-\beta U^{p+1}=0
$$

where each subscript of $\xi$ denotes $\left(\frac{d}{d \xi}\right)$, i.e. differentiation with respect to $\xi$. We define

$$
\begin{aligned}
& y_{1}(\xi)=U(\xi), \\
& y_{2}(\xi)=U_{\xi}(\xi)
\end{aligned}
$$

and thus rewrite (2.9) as a system of two first-order equations

$$
y_{1}^{\prime}=y_{2}, \quad y_{2}^{\prime}=-c y_{2}-\gamma y_{1}+\beta y_{1}^{p+1}
$$

where the primes denote differentiation with respect to $\xi$. The system $(2.10)$ can be written in vector form as $\mathbf{y}^{\prime}=\mathbf{f}(\mathbf{y})$, where $\mathbf{y}=\left(y_{1}, y_{2}\right)$ and $\mathbf{f}$ is the vector valued function given by $\mathbf{f}=\left(f_{1}, f_{2}\right)$ with $f_{1}\left(y_{1}, y_{2}\right)=y_{2}$ and $f_{2}\left(y_{1}, y_{2}\right)=$ $-c y_{2}-\gamma y_{1}+\beta y_{1}^{p+1}$.

The system (2.10) is an example of a nonlinear autonomous system of O.D.Es (A.2). It has one or more critical points which correspond to equilibrium solutions 
of the O.D.E (2.9). If $p$ is an even integer then there are three critical points, $(0,0),\left(\left(\frac{\gamma}{\beta}\right)^{\frac{1}{p}}, 0\right)$ and $\left(-\left(\frac{\gamma}{\beta}\right)^{\frac{1}{p}}, 0\right)$. If $p$ is not even integer, then there are two critical points are $(0,0)$ and $\left(\left(\frac{\gamma}{\beta}\right)^{\frac{1}{p}}, 0\right)$.

We linearize the system (2.10) around each of these critical points and examine the stability of the solution near each critical point. To analyze the stability of solution of the nonlinear system near a critical point $\mathbf{y}_{0}=\left(y_{01}, y_{02}\right)$, we introduce a new variable $\mathbf{z}=\left(z_{1}, z_{2}\right)$ where

$$
z_{1}=y_{1}-y_{01}, \quad z_{2}=y_{2}-y_{02}
$$

Then the critical point corresponds to $\mathbf{z}=(0,0)$ and we can write the nonlinear system as

$$
\mathbf{z}^{\prime}=A \mathbf{z}+\mathbf{g}(\mathbf{z})
$$

where $A$ is a $2 \times 2$ matrix and $\mathbf{g}=\left(g_{1}\left(z_{1}, z_{2}\right), g_{2}\left(z_{1}, z_{2}\right)\right)$ is vector-valued function satisfying $\mathbf{g}(\mathbf{0})=\mathbf{0}$. For each of the critical points of (2.11), we can apply one of the Theorems A.2-A.4 (Appendix A) to determine whether the equilibrium solution is asymptotically stable, unstable or stable.

\section{The critical point $(0,0)$ :}

The nonlinear system (2.10) can be written as $\mathbf{y}^{\prime}=A \mathbf{y}+\mathbf{g}(\mathbf{y})$ with

$$
A=\left(\begin{array}{cc}
0 & 1 \\
-\gamma & -c
\end{array}\right)
$$

and

$$
\begin{gathered}
g_{1}\left(y_{1}, y_{2}\right)=0, \\
g_{2}\left(y_{1}, y_{2}\right)=\beta y_{1}^{p+1} .
\end{gathered}
$$

Linearizing (2.10) around the critical point $(0,0)$ gives

$$
y_{1}^{\prime}=y_{2}, \quad y_{2}^{\prime}=-c y_{2}-\gamma y_{1}
$$


The eigenvalues of the matrix $A$ are given by the characteristic equation

$$
\lambda^{2}+c \lambda+\gamma=0 .
$$

The solutions of (2.15) are

$$
\lambda_{1,2}=\frac{-c \pm \sqrt{c^{2}-4 \gamma}}{2} .
$$

For $c>0$ there are three cases. If $c>2 \sqrt{\gamma}$, then $\lambda_{1}$ and $\lambda_{2}$ are both real and negative and the general solution of the linear system (2.14) is

$$
\mathbf{y}=c_{1} \mathbf{v}_{1} e^{\lambda_{1} \xi}+c_{2} \mathbf{v}_{2} e^{\lambda_{2} \xi},
$$

where $c_{1}$ and $c_{2}$ are arbitrary constants and $\mathbf{v}_{1}$ and $\mathbf{v}_{2}$ are the eigenvectors of $A$ corresponding to $\lambda_{1}$ and $\lambda_{2}$ respectively. From (2.17), $\mathbf{y} \rightarrow \mathbf{0}$ as $\xi \rightarrow \infty$. The critical point $(0,0)$ is a stable node for the linear system. The phase portrait for this case is shown in Figure 2.1 (a) with $\gamma=1, p=1$ and $c=3$ and the eigenvalues are $\lambda_{1,2}=-\frac{2}{\sqrt{6}},-\frac{3}{\sqrt{6}}$.

If $c=2 \sqrt{\gamma}$, there is a repeated eigenvalue $\lambda_{1}=\lambda_{2}<0$. The general solution of the linear system (2.14) is

$$
\mathbf{y}=c_{1} \mathbf{v}_{1} e^{\lambda_{1} \xi}+c_{2}\left(\mathbf{v}_{1} \xi+\mathbf{v}_{2}\right) e^{\lambda_{1} \xi}
$$

where $\mathbf{v}_{1}$ and $\mathbf{v}_{2}$ are generalized eigenvectors and $\mathbf{v}_{1}=\left(A-\lambda I_{2}\right) \mathbf{v}_{2}$ where $I_{2}$ is $2 \times 2$ identity matrix and $\mathbf{y} \rightarrow \mathbf{0}$ as $\xi \rightarrow \infty$. The critical point $(0,0)$ is a stable node for the linear system. The phase portrait for this case is shown in Figure 2.1 (b) with $\gamma=1, p=1$ and $c=2$.

If $0<c<2 \sqrt{\gamma}$, then $\lambda_{1,2}=\frac{-c \pm i \sqrt{4 \gamma-c^{2}}}{2}$ are complex with $\operatorname{Re}\left(\lambda_{1,2}\right)<0$. The general solution of the linear system is

$$
\mathbf{y}=c_{1} \mathbf{v}_{1} e^{-\frac{c}{2} \xi} \cos \left(\frac{\sqrt{4 \gamma-c^{2}}}{2}\right)+c_{2} \mathbf{v}_{2} e^{-\frac{c}{2} \xi} \sin \left(\frac{\sqrt{4 \gamma-c^{2}}}{2}\right)
$$

and $\mathbf{y}$ is oscillatory and approaches $\mathbf{0}$ as $\xi \rightarrow \infty$. The critical point $(0,0)$ is a stable spiral point for the linear system. The phase portrait for this case is shown in Figure 2.1 (c) with $\gamma=1, p=1$ and $c=1$. 
The case with $\gamma=0$ is considered on page 21 .

If $c<0$, there are again there possibilities. If $c<-2 \sqrt{\gamma}$, then $\lambda_{1}$ and $\lambda_{2}$ are both real and positive and the general solution is given by (2.17) and the critical point is an unstable node. If $c=-2 \sqrt{\gamma}$, then $\lambda_{1}=\lambda_{2}>0$ and the general solution is given by (2.18) and critical point is an unstable node. If $-2 \sqrt{\gamma}<c<0$, then $\lambda_{1,2}=\frac{-c \pm i \sqrt{4 \gamma-c^{2}}}{2}$ are complex with $\operatorname{Re}\left(\lambda_{1,2}\right)>0$ and the critical point is an unstable spiral. In each case with $c<0$, the phase portrait looks like one of Figures 2.1 (a) (b) and (c) but with the arrows on the trajectories pointing outwards from the critical point.

If $c=0$, then the solution of (2.7) is independent of time and satisfies the steady equation

$$
\frac{\partial^{2} u}{\partial x^{2}}+u\left(\gamma-\beta u^{p}\right)=0
$$

which can be written as a system of two first-order equations

$$
y_{1}^{\prime}=y_{2}, \quad y_{2}^{\prime}=-\gamma y_{1}+\beta y_{1}^{p+1}
$$

Linearizing around the critical point $(0,0)$ gives $\mathbf{y}^{\prime}=A \mathbf{y}$ with

$$
A=\left(\begin{array}{cc}
0 & 1 \\
-\gamma & 0
\end{array}\right)
$$

and eigenvalues are $\lambda_{1,2}= \pm i \sqrt{\gamma}$ and so Theorem A.1 tells us that the critical point $(0,0)$ is stable for the linear system (2.21) but not asymptotically stable. The general solution of the linear system is $\mathbf{y}=c_{1} \mathbf{v}_{1} \cos (\sqrt{\gamma} \xi)+c_{2} \mathbf{v}_{2} \sin (\sqrt{\gamma} \xi)$ which is oscillatory. The critical point is a center. This case is shown in Figure $2.1(\mathrm{~d})$.

For the nonlinear system (2.10) with $\mathbf{g}(\mathbf{0})=\mathbf{0}, \lim _{|\mathbf{z}| \rightarrow 0} \frac{\mathbf{g}(\mathbf{z})}{|\mathbf{z}|}=0$ and $\lim _{|\mathbf{z}| \rightarrow 0}\left|\frac{\partial \mathbf{g}(\mathbf{z})}{\partial z_{i}}\right|=$ 0 for $i=1,2$. For the case where $c>0$, Theorem A.2 (Appendix A) thus tells us that the critical point $\mathbf{y}=(0,0)$ is asymptotically stable, or the equilibrium solution $y_{1}=u=0$ is asymptotically stable. For the case where $c<0$ Theorem A.3 (Appendix A) tells us that the zero critical point is unstable. 
(a)

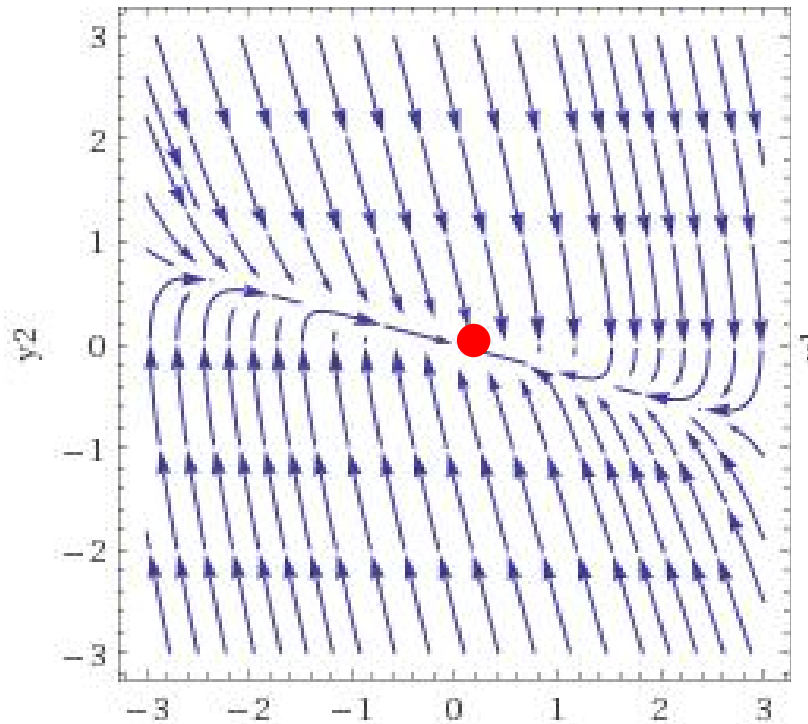

(c)



(b)

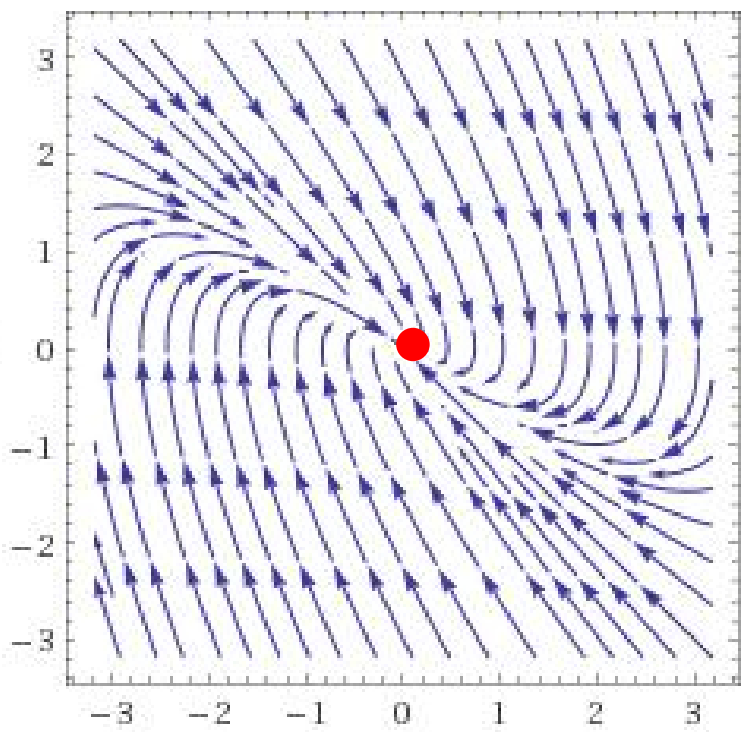

$(d)$

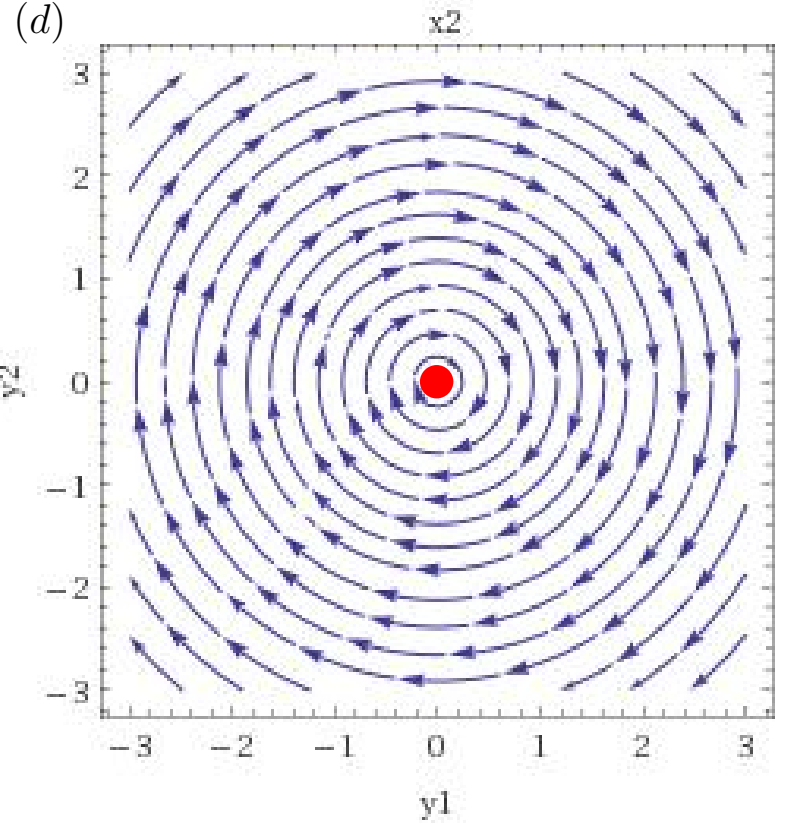

Figure 2.1: Phase portraits for linear system $\mathbf{y}^{\prime}=A \mathbf{y}$, with $A$ given by (2.13), linearized around the critical point $(0,0)$ with $\beta=\gamma=1$. (a) The case where $c=\frac{5}{\sqrt{6}}>2 \sqrt{\gamma}$ and the critical point $\left(y_{1}, y_{2}\right)=(0,0)$ is a stable node. (b) The case where $c=2 \sqrt{\gamma}$, with $\gamma=1, c=2$ and the critical point $\left(y_{1}, y_{2}\right)=(0,0)$ is a stable node. (c) The case where $c=1,0<c<2 \sqrt{\gamma}$ and the critical point $\left(y_{1}, y_{2}\right)=(0,0)$ is a stable spiral. (d) The case where $c=0$ and the critical point $\left(y_{1}, y_{2}\right)=(0,0)$ is a center. 
For the case where $c=0$, we can construct a Lyapunov function $V\left(y_{1}, y_{2}\right)$ as defined in Appendix A and then apply Theorem A.4 to prove that the critical point $(0,0)$ is stable for the nonlinear system $(2.21)$ we define

$$
y_{1}^{\prime}=y_{2}=f_{1}\left(y_{1}, y_{2}\right), \quad y_{2}^{\prime}=-c y_{2}-\gamma y_{1}+\beta y_{1}^{p+1}=f_{2}\left(y_{1}, y_{2}\right) .
$$

Let

$$
V\left(y_{1}, y_{2}\right)=\frac{y_{2}^{2}}{2}+\int_{0}^{y_{1}}\left(\gamma \sigma-\beta \sigma^{p+1}\right) d \sigma=\frac{y_{2}^{2}}{2}+\frac{\gamma y_{1}^{2}}{2}-\frac{\beta y_{1}^{p+2}}{p+2},
$$

which is positive definite in the region $\Omega=\left\{\left(y_{1}, y_{2}\right):\left|y_{1}\right|<\left(\frac{\gamma}{\beta}\right)^{\frac{1}{p}},\left|y_{2}\right|<\infty\right\}$. According to Definition A.8 in Appendix A, the derivative of $V$ with respect to the system $(2.21)$ is

$$
V^{*}(\mathbf{y})=\nabla V(\mathbf{y}) \cdot \mathbf{f}(\mathbf{y})
$$

where $\mathbf{f}(\mathbf{y})=\left(f_{1}\left(y_{1}, y_{2}\right), f_{2}\left(y_{1}, y_{2}\right)\right)=\left(y_{2},-c y_{2}-\gamma y_{1}+\beta y_{1}^{p+1}\right)$. We see that

$$
V^{*}(\mathbf{y})=\left(\gamma y_{1}-\beta y_{1}^{p+1}\right) y_{2}+y_{2}\left(-c y_{2}-\gamma y_{1}+\beta y_{1}^{p+1}\right)=-c y_{2}^{2} \leq 0 .
$$

Thus, the zero solution of the nonlinear system is stable according to Theorem A.4.

The critical point $\left(\left(\frac{\gamma}{\beta}\right)^{\frac{1}{p}}, 0\right)$ :

To analyze the critical point $\mathbf{y}_{0}=\left(\left(\frac{\gamma}{\beta}\right)^{\frac{1}{p}}, 0\right)$, we define $\mathbf{z}=\left(z_{1}, z_{2}\right)$ according to (2.11), by

$$
z_{1}=y_{1}-\left(\frac{\gamma}{\beta}\right)^{\frac{1}{p}}, \quad z_{2}=y_{2}-0
$$

to obtain the system of equations

$$
z_{1}^{\prime}=z_{2}, \quad z_{2}^{\prime}=-c z_{2}-\gamma\left\{z_{1}+\left(\frac{\gamma}{\beta}\right)^{\frac{1}{p}}\right\}+\beta\left\{z_{1}+\left(\frac{\gamma}{\beta}\right)^{\frac{1}{p}}\right\}^{p+1} .
$$

Using the binomial theorem, the equation for $z_{2}$ can be written as

$$
z_{2}^{\prime}=-c z_{2}-\gamma z_{1}+\gamma(p+1) z_{1}+\sum_{k=0}^{p-1} \frac{(p+1) !}{k !(p+1-k) !}\left(\frac{\gamma}{\beta}\right)^{\frac{k}{p}} z_{1}^{p+1-k}
$$


The nonlinear system (2.23) can then be written in the form (2.12) with

$$
A=\left(\begin{array}{cc}
0 & 1 \\
p \gamma & -c
\end{array}\right)
$$

and

$$
\begin{gathered}
g_{1}\left(z_{1}, z_{2}\right)=0 \\
g_{2}\left(z_{1}, z_{2}\right)=\sum_{k=0}^{p-1} \frac{(p+1) !}{k !(p+1-k) !}\left(\frac{\gamma}{\beta}\right)^{\frac{k}{p}} z_{1}^{p+1-k}
\end{gathered}
$$

and can linearized to give by $\mathbf{y}^{\prime}=A \mathbf{y}$ with $A$ given by (2.24). The characteristic equation for $A$ is

$$
\lambda^{2}+c \lambda-p \gamma=0
$$

and thus the eigenvalues are

$$
\lambda_{1,2}=\frac{1}{2}\left(-c \pm \sqrt{c^{2}+4 p \gamma}\right)
$$

We observe that $\lambda_{1}<0$ and $\lambda_{2}>0$, so the critical point $\left(\left(\frac{\gamma}{\beta}\right)^{\frac{1}{p}}, 0\right)$ is a saddle point for the linear system. The solution of the linear system is $\mathbf{y}=c_{1} \mathbf{v}_{1} e^{\lambda_{1} \xi}+$ $c_{2} \mathbf{V}_{2} e^{\lambda_{2} \xi}$.

Figure 2.2 shows the phase portrait for the linearized system around this critical point for the case where $p=2, \beta=1$ and $\gamma=1$. The critical point is at $(1,0)$ and the equilibrium solution is $u=1$. The line passing through the critical point in the direction of the eigenvector $\mathbf{v}_{1}$, which corresponds to the negative eigenvalue, is the stable manifold for the critical point, i.e., any solution starting at a point on this line approaches the equilibrium solution as $\xi \rightarrow \infty$. The line passing through the critical point in the direction of the eigenvector $\mathbf{v}_{2}$, which corresponds to the positive eigenvalue, is the unstable manifold, i.e., any solution starting at a point on this line grows exponentially as $\xi \rightarrow \infty$.

For the nonlinear system $(2.23), \mathbf{g}(\mathbf{0})=\mathbf{0}$. Thus, $\lim _{|\mathbf{z}| \longrightarrow o}\left|\frac{\partial \mathbf{g}(\mathbf{z}))}{\partial z_{i}}\right|=0$ and so the zero solution is unstable according to Theorem A.3 (Appendix A). 


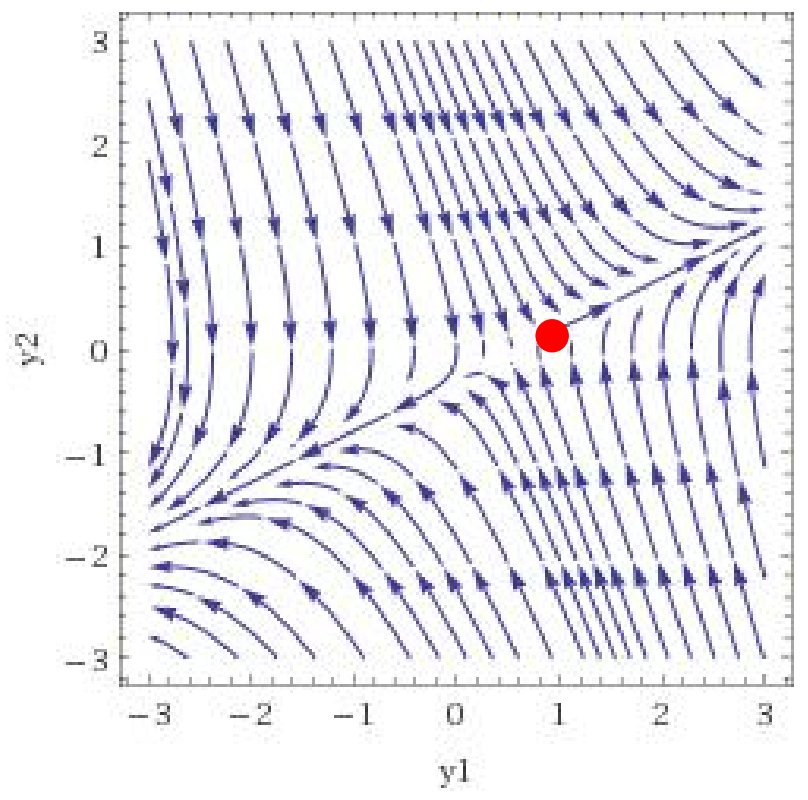

Figure 2.2: Phase portrait for the linear system $\mathbf{y}^{\prime}=A \mathbf{y}$ with $A$ given by (2.32), linearized around the critical point $(1,0)$ with $p=\gamma=\beta=1$. This point is a saddle point for the linear system. 
The critical point $\left(-\left(\frac{\gamma}{\beta}\right)^{\frac{1}{p}}, 0\right)$ for $p$ even:

If $p$ is even, then there is an additional critical point $\left(-\left(\frac{\gamma}{\beta}\right)^{\frac{1}{p}}, 0\right)$. To analyze the critical point $\mathbf{y}_{0}=\left(-\left(\frac{\gamma}{\beta}\right)^{\frac{1}{p}}, 0\right)$, we define $\mathbf{z}=\left(z_{1}, z_{2}\right)$ according to (2.11).

We write equation (2.14) in the form (2.10) and define $\mathbf{z}=\left(z_{1}, z_{2}\right)$, according to $(2.11)$, by

$$
z_{1}=y_{1}+\left(\frac{\gamma}{\beta}\right)^{\frac{1}{p}}, \quad z_{2}=y_{2}-0
$$

to obtain

$$
z_{1}^{\prime}=z_{2} \quad z_{2}^{\prime}=-c z_{2}-\gamma\left\{z_{1}-\left(\frac{\gamma}{\beta}\right)^{\frac{1}{p}}\right\}+\beta\left\{z_{1}-\left(\frac{\gamma}{\beta}\right)^{\frac{1}{p}}\right\}^{p+1} .
$$

Using the binomial theorem, the equation for $z_{2}$ can be written as

$$
\begin{aligned}
z_{2}^{\prime}=-c z_{2}-\gamma z_{1}+\gamma\left(\frac{\gamma}{\beta}\right)^{\frac{1}{p}}+ & (-1)^{p} \gamma(p+1) z_{1}+(-1)^{p+1} \gamma\left(\frac{\gamma}{\beta}\right)^{\frac{1}{p}} \\
& +\sum_{k=0}^{p-1} \frac{(p+1) !}{k !(p+1-k) !}\left(\frac{\gamma}{\beta}\right)^{\frac{k}{p}}(-1)^{k} z_{1}^{p+1-k}
\end{aligned}
$$

The nonlinear system can be written in the form (2.12) with

$$
A=\left(\begin{array}{cc}
0 & 1 \\
p \gamma & -c
\end{array}\right)
$$

and

$$
\begin{gathered}
g_{1}\left(z_{1}, z_{2}\right)=0 \\
g_{2}\left(z_{1}, z_{2}\right)=\sum_{k=0}^{p-1} \frac{(p+1) !}{(k ! p+1-k) !}\left(\frac{\gamma}{\beta}\right)^{\frac{k}{p}}(-1)^{k} z_{1}^{p+1-k}
\end{gathered}
$$

and can be linearized to give by $\mathbf{y}^{\prime}=A \mathbf{y}$ with $A$ given by (2.24). The characteristic equation for $A$ is

$$
\lambda^{2}+c \lambda-p \gamma=0
$$

and thus the eigenvalues are 


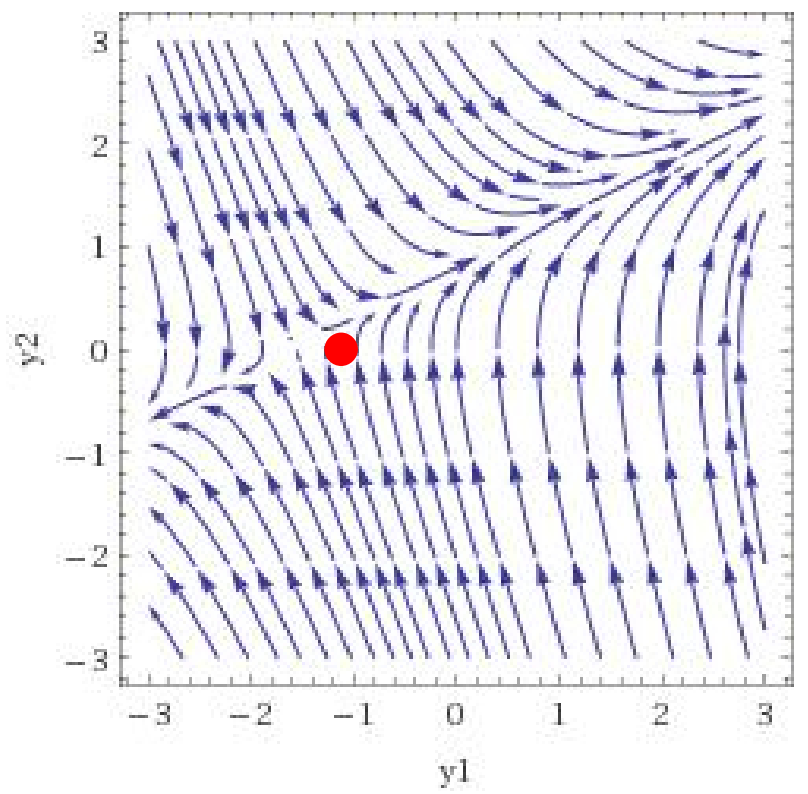

Figure 2.3: Phase portrait for the linear system $\mathbf{y}^{\prime}=A \mathbf{y}$ with $A$ given by (2.34), linearized around the saddle point $(-1,0)$ with $\gamma=\beta=1$ and $p=2$.

$$
\lambda_{1,2}=\frac{1}{2}\left(-c \pm \sqrt{c^{2}+4 p \gamma}\right)=0 .
$$

We observe that $\lambda_{1}<0$ and $\lambda_{2}>0$, so the point $\left(-\left(\frac{\gamma}{\beta}\right)^{\frac{1}{p}}, 0\right)$ is a saddle point for the linearized system. The solution of the linear system is $\mathbf{y}=c_{1} \mathbf{v}_{1} e^{\lambda_{1} \xi}+c_{2} \mathbf{v}_{2} e^{\lambda_{2} \xi}$. Figure 2.3 shows the phase portrait for the linearized system around this critical point for the case where $p=2$ and $\beta=\gamma=1$. The critical point is at $(-1,0)$ and the equilibrium solution is $u=-1$. As before, the line passing through the critical point in the direction of the eigenvector $\mathbf{v}_{1}$, which corresponds to the negative eigenvalue, is the stable manifold for the critical point and the line in the direction of the eigenvector $\mathbf{v}_{2}$, which corresponds to the positive eigenvalue, is the unstable manifold.

For the nonlinear system $(2.24), \mathbf{g}(\mathbf{0})=\mathbf{0}$ and $\lim _{|\mathbf{z}| \longrightarrow o}\left|\frac{\partial \mathbf{g}(\mathbf{z}))}{\partial z_{i}}\right|=0$. Thus, the zero solution is an unstable according to Theorem A.3 (Appendix A). Figures 2.4, 2.5 and 2.6, show the phase portrait for the nonlinear system (2.10) with 
$\beta=\gamma=1$

In Figure 2.4 (a), $c=3>2 \sqrt{\gamma}$. The saddle point $(1,0)$ has a stable manifold represented by the two trajectories that approach this point as $\xi \rightarrow \infty$. All the trajectories on the left approach the stable node and theses on the right go to infinity as $\xi \rightarrow \infty$. In Figure $2.4(\mathrm{~b}), c=0$ and the point $(0,0)$ is a center. The solutions around this point are oscillatory. This a homoclinic orbit which joins the saddle point $(1,0)$ to itself.

Figure 2.5 (a) shows the same phase portrait as 2.4 (a) with $c=3$, but enlarged to show more details around the critical points. Figure 2.5 (b) shows the corresponding phase portrait with $c=2$. In both cases, there is a heteroclinic orbit from the saddle point $(1,0)$ to the stable node $(0,0)$ lying in the region where $0<y_{1}=U<1$ and $y_{2}=U_{\xi}<0$. This corresponds to a travelling wave solution which satisfies $U \rightarrow 1$ as $\xi \rightarrow-\infty$ and $U \rightarrow 0$ as $\xi \rightarrow \infty$ with $U_{\xi}<0$. This solution preserves non-negatively. These two Figure 2.5 (a) and 2.5 (b), illustrate the situation where there is a travelling wave solution of equation (1.5) with (1.2). Figure $2.5(\mathrm{c})$ shows the case where $c=1<2 \sqrt{\gamma}$. In this case, critical point $(0,0)$ is a spiral point and the solutions around this point are oscillatory and do not preserve non-negatively. Figure 2.5 (b), $0>c=-3>-2 \sqrt{\gamma}$. In this case, there is a heteroclinic orbit from $(0,0)$ to $(1,0)$ which corresponds to a travelling wave solution satisfying $U \rightarrow 0$ as $\xi \rightarrow-\infty$ and $U \rightarrow 1$ as $\xi \rightarrow \infty$ with $U_{\xi}>0$. In summary, the condition for a travelling wave solution (which preserves nonnegativity) is that $|c| \geq 2 \sqrt{\gamma}$. If $c>0$, the wave travels to the right and if $c<0$ it travels to the left.

In Figure 2.6, $p=2$ and there is a stable node at $(0,0)$ and two saddle points at $(1,0)$ and $(-1,0)$. In Figure $2.6(\mathrm{a}), c=3>2 \sqrt{\gamma}$. There is two hereoclinic orbits, one going from the saddle point $(1,0)$ to the stable node and other going from the saddle point $(-1,0)$ to the stable node. These correspond to travelling wave solutions. In Figure $2.6(\mathrm{~b}), c=0$ and the point $(0,0)$ is center. The solutions 
around this point are oscillatory.

\section{The case where $\gamma=0$}

Finally, we consider the case where $\gamma=0$ in (2.7). Setting $\bar{c}=0$ gives

$$
\frac{\partial u}{\partial t}=\frac{\partial^{2} u}{\partial x^{2}}-\beta u^{p+1}, \quad p>0 .
$$

The substitution (2.8) gives

$$
U_{\xi \xi}+c U_{\xi}-\beta U^{p+1}=0,
$$

which can be written as a system of two first order equations

$$
y_{1}^{\prime}=y_{2}, \quad y_{2}^{\prime}=-c y_{2}+\beta y_{1}^{p+1}
$$

or in vector matrix form as $\mathbf{y}^{\prime}=A \mathbf{y}+\mathbf{g}(\mathbf{y})$ with

$$
\begin{gathered}
A=\left(\begin{array}{cc}
0 & 1 \\
0 & -c
\end{array}\right), \\
g_{1}\left(y_{1}, y_{2}\right)=0, \quad g_{2}\left(y_{1}, y_{2}\right)=\beta y_{1}^{p+1} .
\end{gathered}
$$

The eigenvalues of the matrix $A$ are given by the characteristic equation

$$
\lambda^{2}+c \lambda=0 .
$$

The eigenvalues are

$$
\lambda_{1}=0 \text { and } \lambda_{2}=-c .
$$

The general solution of the linear system $\mathbf{y}^{\prime}=A \mathbf{y}$ is $\mathbf{y}=c_{1} \mathbf{v}_{1}+c_{2} \mathbf{v}_{2} e^{-c \xi}$ where $c_{1}$ and $c_{2}$ are arbitrary constants and $\mathbf{v}_{1}$ and $\mathbf{v}_{2}$ are the eigenvectors of $A$ corresponding to $\lambda_{1}$ and $\lambda_{2}$, respectively. As $\xi \rightarrow \infty$ the solution $\mathbf{y} \rightarrow c_{1} \mathbf{v}_{1}$ along a line parallel to $\mathbf{v}_{2}$ as $\xi \rightarrow \infty$. The zero eigenvalue means that the linear system $\mathbf{y}=A \mathbf{y}$, which corresponds to $\beta=0$ in (2.27), has infinitely many critical 



Figure 2.4: Phase portraits for the nonlinear system (2.14) with $\beta=\gamma=1$. (a) The case where $c=3>2 \sqrt{\gamma}$. This corresponds to the Fisher equation (1.4). (b) The case where $c=0$. The critical points are $(0,0)$ and $(1,0)$. 
(a)
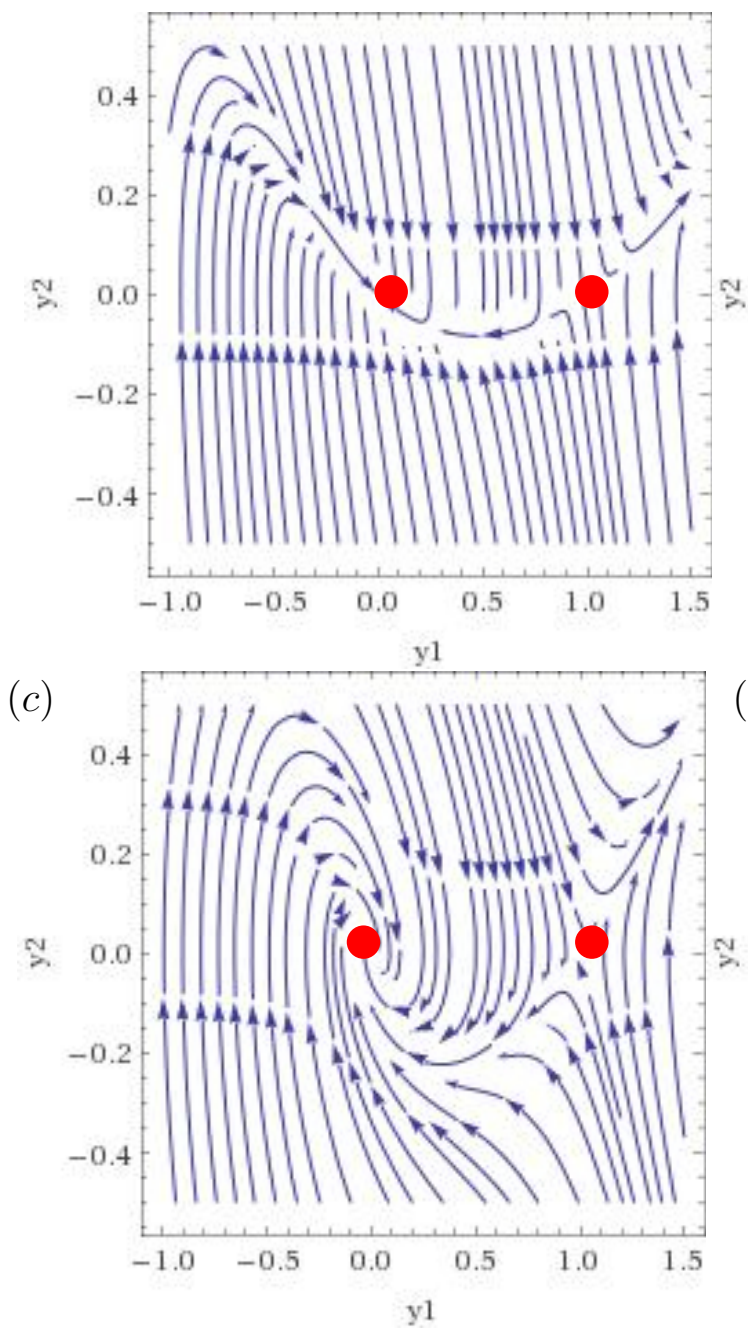

(b)

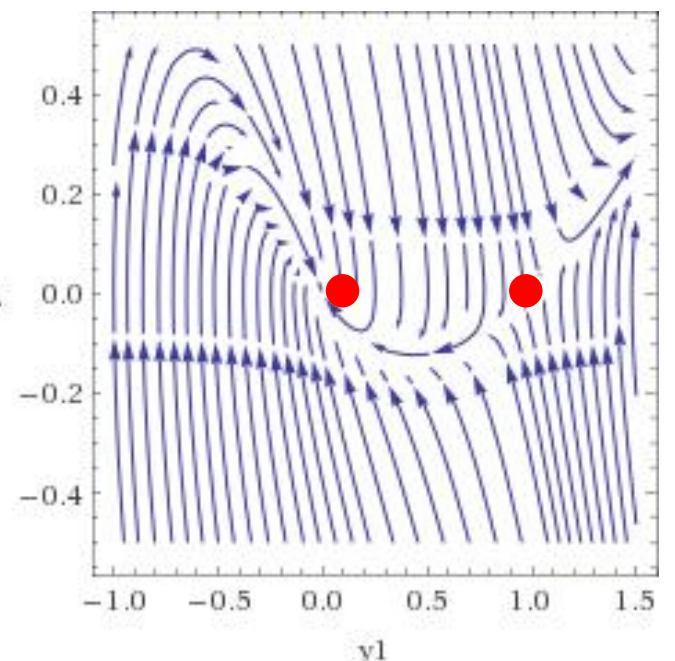

$(d)$

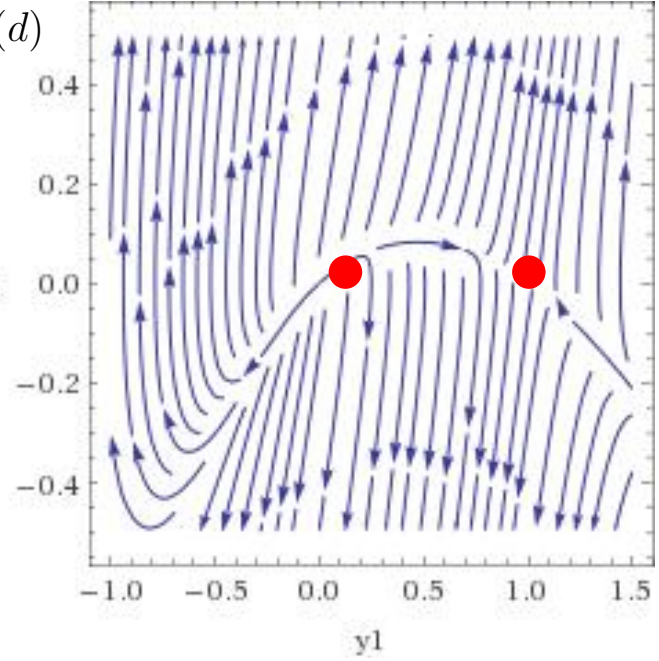

Figure 2.5: Phase portraits for the nonlinear system (2.14) with $\beta=\gamma=1$. This corresponds to the Fisher equation (1.4). The critical points are $(0,0)$ and $(1,0)$ (a) The case where $c=3>2 \sqrt{\gamma}$, the same case as Figure 2.4 (a) but enlarged. There is a heteroclinic orbit (a trajectory which joins two different equilibrium points) from $(1,0)$ to $(0,0)$. (b) The case where $c=2=2 \sqrt{\gamma}$. There is a heteroclinic orbit from $(1,0)$ to $(0,0)$ (c) The case where $0<c=1<2 \sqrt{\gamma}$. (d) The case where $-2 \sqrt{\gamma}<c=-3<0$. 
(a)

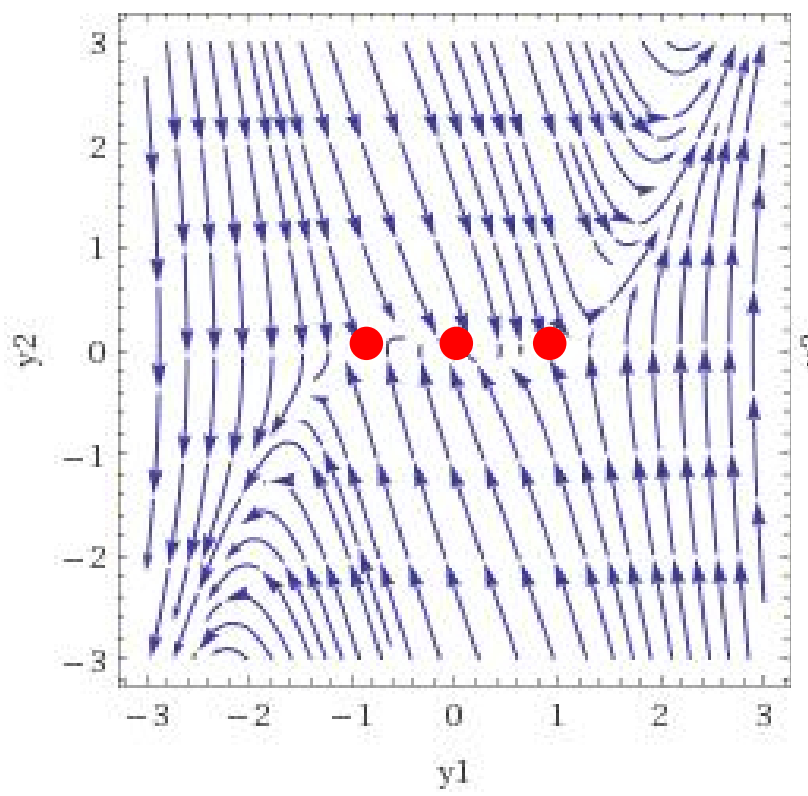

(b)

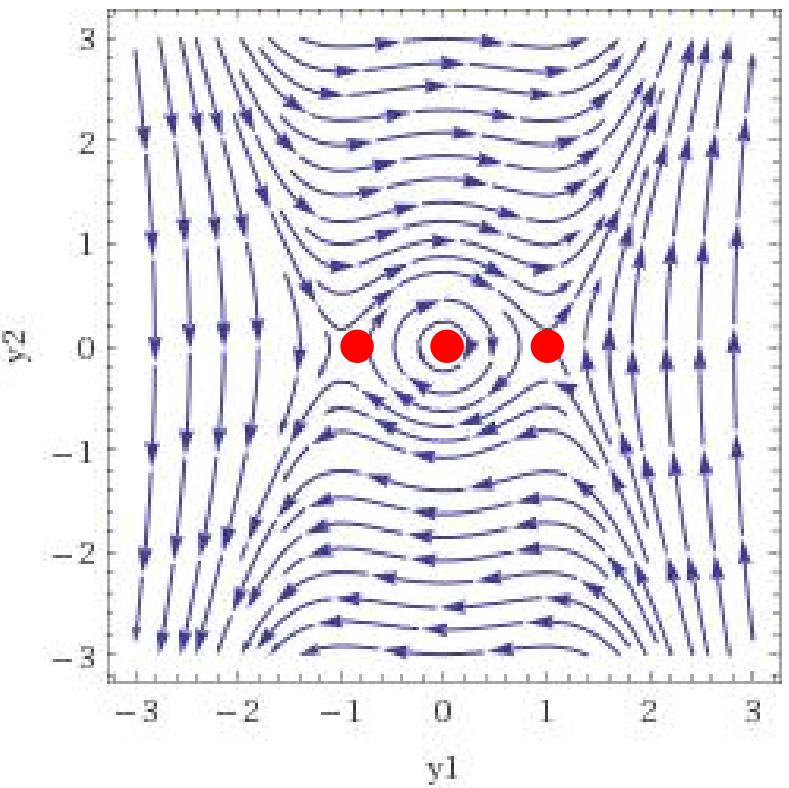

Figure 2.6: Phase portraits for the nonlinear system (2.31) with $\beta=0$ and $\alpha=$ $\gamma=1$. The critical points are $(0,0)$ and $( \pm 1,0)$. (a) The case where $c=3>2 \sqrt{\gamma}$. (b) The case where $c=0$.

points corresponding to the line $y_{2}=0$ and the trajectories are lines with slope $\frac{d y_{2}}{d y_{1}}=-c$. The phase portrait for the linear system is shown in Figure 2.7 (a). With $c>0$ the critical points are stable and the arrows on the trajectories point towards from the line $y_{2}=0$ as shown in the figure. With $c<0$ the critical points are unstable and the arrows on the trajectories would point away from the line $y_{2}=0$.

For the nonlinear system (2.27) with $\beta \neq 0$, there is only one critical point at $(0,0)$. Phase portraits for the nonlinear system $(2.27)$ are shown in Figures 2.7 (b) (c) and (d) for $c>0$ and for different values of $\beta$. 
$(a)$
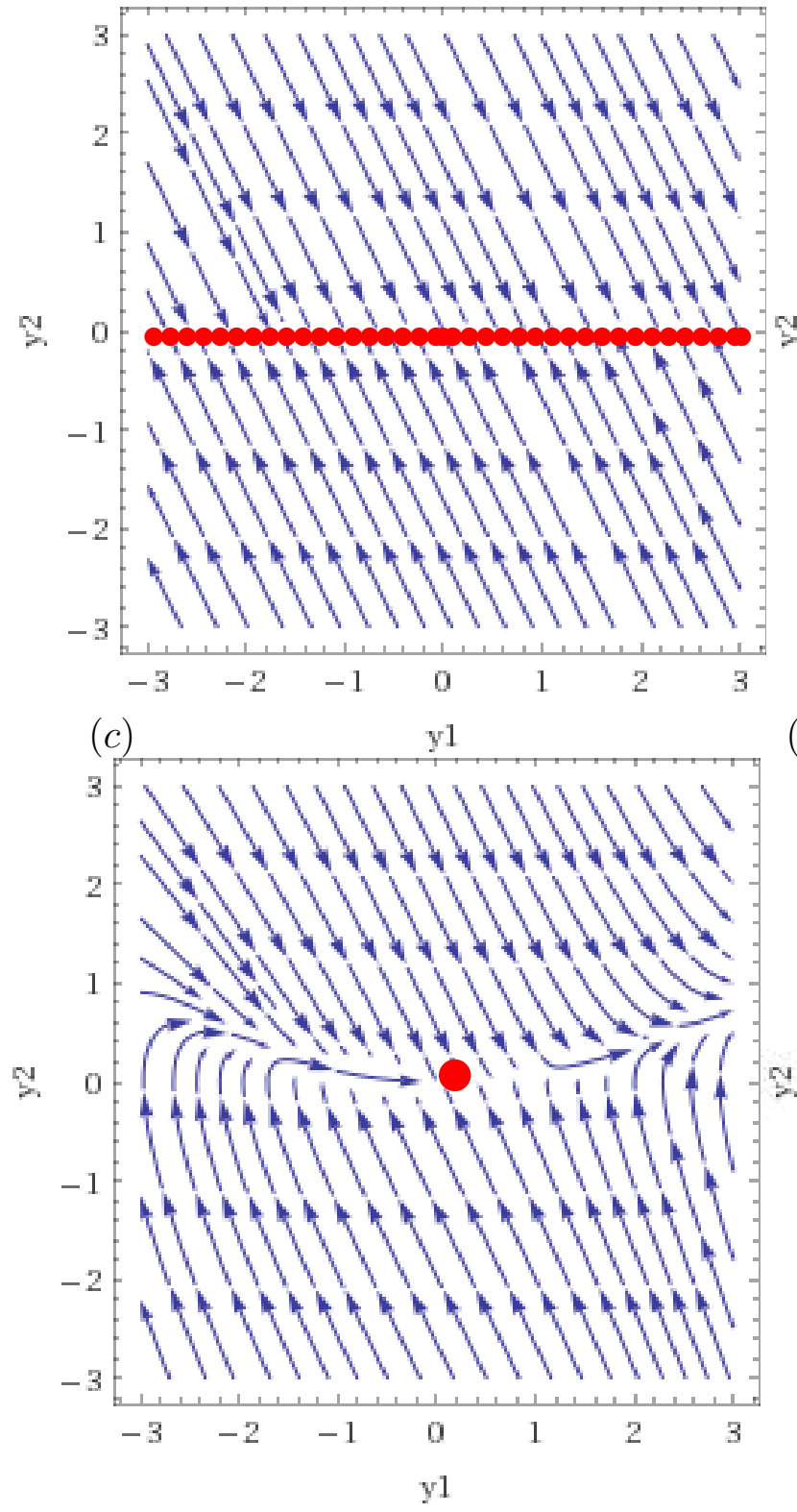

$(b)$

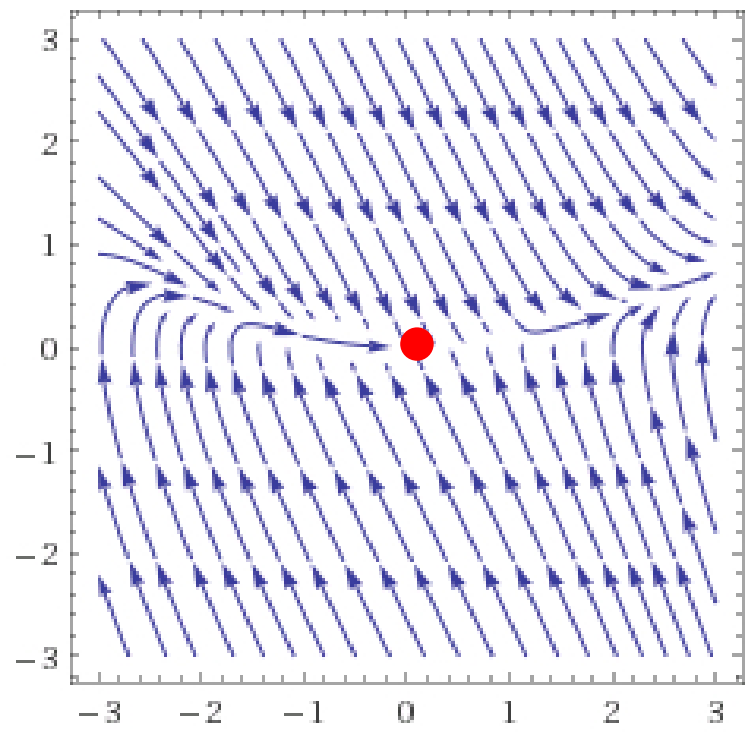

(d)

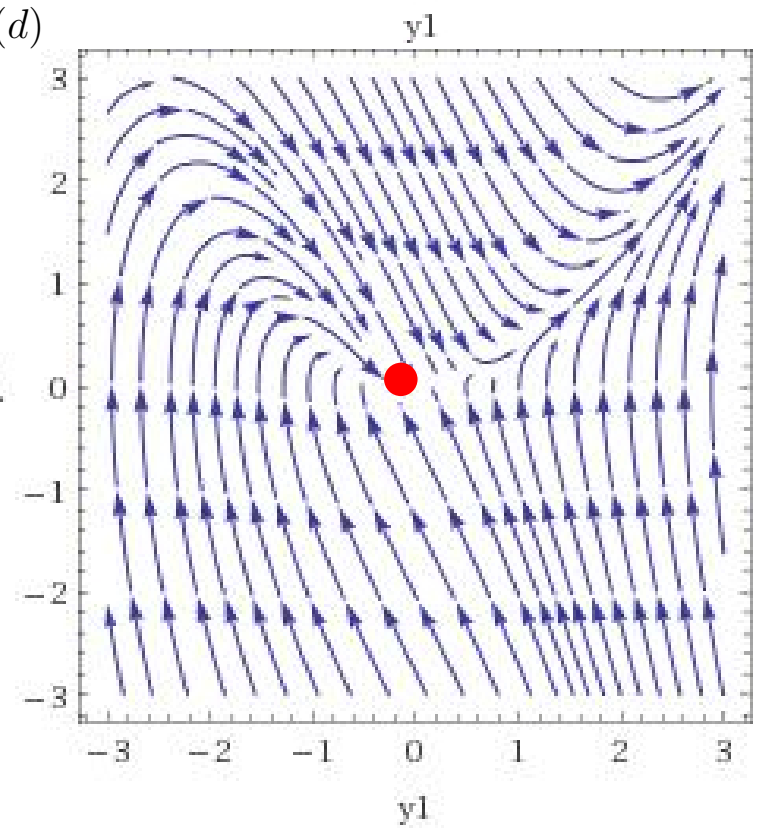

Figure 2.7: Phase portraits for the nonlinear system for the case where $\gamma=0$, (2.27) with $c=3$ and $p=1$ (a) $\beta=0$ (linear system), (b) $\beta=0.03$, (c) $\beta=0.2$, (d) $\beta=1$. 


\subsection{Stability of the Equilibrium Solutions for the Case with General $\alpha, \beta$ and $\gamma$}

In this section we consider (2.6) with $\bar{c}$ and $\alpha \neq 0$. If $\alpha=0$, we obtain the equation from section 2.2 with $p=1$, which has already been discussed in section 2.2 .

As before, we seek a solution of the form (2.8). This gives nonlinear autonomous O.D.E

$$
U_{\xi \xi}+c U_{\xi}-\alpha U^{3}-\beta U^{2}+\gamma U=0 .
$$

To determine the critical points we define

$$
\begin{aligned}
& y_{1}(\xi)=U(\xi), \\
& y_{2}(\xi)=U_{\xi}(\xi)
\end{aligned}
$$

and thus rewrite (2.30) as a system of two first-order equations

$$
y_{1}^{\prime}=y_{2} \quad y_{2}^{\prime}=-c y_{2}+\alpha y_{1}^{3}+\beta y_{1}^{2}-\gamma y_{1}
$$

where the primes denote differentiation with respect to $\xi$. The system $(2.31)$ can be written in vector form as $\mathbf{y}^{\prime}=\mathbf{f}(\mathbf{y})$, where $\mathbf{y}=\left(y_{1}, y_{2}\right)$ and $\mathbf{f}$ is the vector valued function given by $\mathbf{f}=\left(f_{1}, f_{2}\right)$ with $f_{1}\left(y_{1}, y_{2}\right)=y_{2}$ and $f_{2}\left(y_{1}, y_{2}\right)=$ $-c y_{2}+\alpha y_{1}^{3}+\beta y_{2}-\gamma y_{2}$. The system (2.31) is an example of a nonlinear autonomous system of O.D.Es (A.2). It has three critical points.

The critical points of $(2.31)$ are $(0,0),\left(\frac{-\beta \pm \sqrt{\beta^{2}+4 \alpha \gamma}}{2 \alpha}, 0\right)$. These correspond to the equilibrium solutions $u=y_{1}=0$ and $u=y_{1}=\frac{-\beta \pm \sqrt{\beta^{2}+4 \alpha \gamma}}{2 \alpha}$, one of which is positive and the other negative. We linearize around each critical points.

\section{The critical point $(0,0)$ :}

Linearizing around the critical point $(0,0)$ gives the same linear system as in section 2.2, $\mathbf{y}^{\prime}=A \mathbf{y}$ with A given by (2.13). For $c \geq 2 \sqrt{\gamma}$ the critical point is 
stable node, for the case $0<c<2 \sqrt{\gamma}$ it is a stable spiral point, for $c \leq-2 \sqrt{\gamma}$ it is unstable node, for $-2 \sqrt{\gamma}<c<0$ it is an unstable spiral point and $c=0$ corresponding to the time independent problem. The linear phase portraits are the same as these given in Figure 2.1.

For the nonlinear system (2.31) with $\mathbf{g}(\mathbf{0})=0, \lim _{|\mathbf{z}| \rightarrow 0} \frac{\mathbf{g}(\mathbf{z})}{|\mathbf{z}|}=0$ and $\lim _{|\mathbf{z}| \rightarrow 0}\left|\frac{\partial \mathbf{g}(\mathbf{z})}{\partial z_{i}}\right|=$ 0 for $i=1,2$. If $c>0$, Theorem A.2 (Appendix A) tells us that the zero critical point $\mathbf{y}=(0,0)$ is asymptotically stable, the equilibrium solution $y_{1}=u=0$ asymptotically stable. If $c<0$, Theorem A.3 (Appendix A) tells us that the zero critical point is unstable.

If $c=0$, we can construct a Lyapunov function as done in section 2.2 and then apply Theorem A.4 to prove that the zero critical point is stable.

\section{The critical point $\left(\frac{-\beta+\sqrt{\beta^{2}+4 \alpha \gamma}}{2 \alpha}, 0\right)$ :}

To analyze the critical point $\mathbf{y}_{0}=\left(\frac{-\beta+\sqrt{\beta^{2}+4 \alpha \gamma}}{2 \alpha}, 0\right)$, we define $\mathbf{z}=\left(z_{1}, z_{2}\right)$ by according to (2.11).

The nonlinear system (2.31) can be then written in the form (2.12) with

$$
A=\left(\begin{array}{cc}
0 & 1 \\
\frac{\beta^{2}}{2 \alpha}-\frac{\beta \sqrt{\beta^{2}+4 \alpha \gamma}}{2 \alpha}+2 \gamma & -c
\end{array}\right)
$$

and

$$
\begin{gathered}
g_{1}\left(z_{1}, z_{2}\right)=0 \\
g_{2}\left(z_{1}, z_{2}\right)=\alpha z_{1}^{3}-3 \alpha z^{2} \frac{\beta+\sqrt{\beta^{2}+4 \alpha \gamma}}{2 \alpha}+\beta z_{1}^{2}-2 \beta z_{1}\left(\frac{-\beta+\sqrt{\beta^{2}+4 \alpha \gamma}}{2 \alpha}\right) \\
-\alpha\left(\frac{-\beta+\sqrt{\beta^{2}+4 \alpha \gamma}}{2 \alpha}\right)^{3}+\beta\left(\frac{-\beta+\sqrt{\beta^{2}+4 \alpha \gamma}}{2 \alpha}\right)^{2}+\gamma\left(\frac{-\beta+\sqrt{\beta^{2}+4 \alpha \gamma}}{2 \alpha}\right) .
\end{gathered}
$$

and can be linearized to give $\mathbf{y}^{\prime}=A \mathbf{y}$ with $A$ given by (2.32). The characteristic equation for $A$ is

$$
\lambda^{2}+c \lambda-\left(\frac{\beta^{2}}{2 \alpha}-\frac{\beta \sqrt{\beta^{2}+4 \alpha \gamma}}{2 \alpha}+2 \gamma\right)=0
$$


and thus the eigenvalues are

$$
\lambda_{1,2}=\frac{1}{2}\left(-c \pm \sqrt{c^{2}+\frac{2 \beta^{2}}{\alpha}-\frac{2 \beta \sqrt{\beta^{2}+4 \alpha \gamma}}{\alpha}+8 \gamma}\right)=0 .
$$

We observe that $\lambda_{1}>0$ and $\lambda_{2}<0$ since

$$
c^{2}+\frac{2 \beta^{2}}{\alpha}-\frac{2 \beta \sqrt{\beta^{2}+4 \alpha \gamma}}{\alpha}+8 \gamma=c^{2}+\frac{2 \sqrt{\beta^{2}+4 \alpha \gamma}}{\alpha}\left(\sqrt{\beta^{2}+4 \alpha \beta}-\beta\right)>c .
$$

and so the critical point $y_{0}=\left(\frac{-\beta+\sqrt{\beta^{2}+4 \alpha \gamma}}{2 \alpha}, 0\right)$ is a saddle point for the linear system. Figure 2.2 shows the phase portrait for the linearzed system around this critical point for the case where $\alpha=1, \beta=0$ and $\gamma=1$. The critical point is at $(1,0)$ and the equilibrium solution is $u=1$. This is the case discussed in section 2.2 with $p=2$.

For the nonlinear system (2.31) written in the form (2.12), $\mathbf{g}(\mathbf{0})=\mathbf{0}$ and $\lim _{|\mathbf{z}| \rightarrow 0}=\left|\frac{\partial \mathbf{g}(\mathbf{z})}{\partial z_{i}}\right|=0(i=1,2)$ and thus the zero critical point $\mathbf{z}=0$ is unstable according to Theorem A.3 (Appendix A). This means that the critical point $\mathbf{y}=\mathbf{y}_{0}$ is unstable.

\section{The critical point $\left(\frac{-\beta-\sqrt{\beta^{2}+4 \alpha \gamma}}{2 \alpha}, 0\right)$ :}

To analyze the critical point $\mathbf{y}_{0}=\left(\frac{-\beta-\sqrt{\beta^{2}+4 \alpha \gamma}}{2 \alpha}, 0\right)$, we define $\mathbf{z}=\left(z_{1}, z_{2}\right)$ according to (2.11). The nonlinear system (2.31) can be then written in the form (2.12) with

$$
\begin{gathered}
A=\left(\begin{array}{cc}
0 & 1 \\
\frac{\beta^{2}}{2 \alpha}+\frac{\beta \sqrt{\beta^{2}+4 \alpha \gamma}}{2 \alpha}+2 \gamma & -c
\end{array}\right) \\
g_{1}\left(z_{1}, z_{2}\right)=0 \\
g_{2}\left(z_{1}, z_{2}\right)=\alpha z_{1}^{3}-3 \alpha z^{2} \frac{\beta+\sqrt{\beta^{2}+4 \alpha \gamma}}{2 \alpha}+\beta z^{2}\left(\frac{\beta+\sqrt{\beta^{2}+4 \alpha \gamma}}{2 \alpha}\right)\left(-\frac{\beta^{2}}{4 \alpha}\right. \\
\left.-\frac{\beta \sqrt{\beta^{2}+4 \alpha \gamma}}{2 \alpha}-\frac{\beta^{2}}{4 \alpha}-\gamma+\frac{\beta^{2}}{2 \alpha}+\frac{\gamma \sqrt{\beta^{2}+4 \alpha \gamma}}{2 \alpha}+\gamma\right)
\end{gathered}
$$

and can be linearized to give $\mathbf{y}^{\prime}=A \mathbf{y}$ with $A$ given by (2.34). The characteristic equation for $A$ is 


$$
\lambda^{2}+c \lambda-\left(\frac{\beta^{2}}{2 \alpha}+\frac{\beta \sqrt{\beta^{2}+4 \alpha \gamma}}{2 \alpha}+2 \gamma\right)=0
$$

and thus the eigenvalues are

$$
\lambda_{1,2}=\frac{1}{2}\left(-c \pm \sqrt{c^{2}+4\left(\frac{\beta^{2}}{2 \alpha}+\frac{\beta \sqrt{\beta^{2}+4 \alpha \gamma}}{2 \alpha}+2 \gamma\right.}\right)=0 .
$$

We observe that $\lambda_{1}>0$ and $\lambda_{2}<0$ and so the point $\mathbf{y}_{0}=\left(\frac{-\beta+\sqrt{\beta^{2}+4 \alpha \gamma}}{2 \alpha}, 0\right)$ is a saddle point for the linear system. Figure 2.3 shows the phase portrait at the point around this critical point for the case where $\alpha=1, \beta=0$ and $\gamma=1$. The critical point is at $(-1,0)$ and the equilibrium solution is $u=-1$. This is the case discussed in section 2.2 with $p=2$.

For the nonlinear system (2.31) written in the form $(2.12), \mathbf{g}(\mathbf{0})=\mathbf{0}$ and $\lim _{|\mathbf{z}| \rightarrow 0}\left|\frac{\partial \mathbf{g}(\mathbf{z})}{\partial z_{i}}\right|=0,(i=1,2)$ and thus the zero solution $\mathbf{z}$ is unstable according to Theorem A.3 (Appendix A). This means that the critical point $\mathbf{y}=\mathbf{y}_{0}$ is unstable.

The phase portraits for the nonlinear system (2.31) with $\gamma \neq 0$ would look like the phase portrait in Figure 2.6 (a) for $c>2 \sqrt{\gamma}$ and like the phase portrait2.6 (b) for $c=0$. 


\section{Chapter 3}

\section{Derivation of Travelling Wave Solutions}

\subsection{Introduction}

In this chapter we derive travelling wave solutions some special cases for the nonlinear reaction-diffusion equation (1.1) with the nonlinear function given by (1.2). This equation is written in nondimensional form as (2.7). For simplicity, we consider the case $\bar{c}=0$. If $\bar{c} \neq 0$, then we obtain the same solutions but with the value of $c$ changed by an increment of $\bar{c}$. The special case where $\beta=1, \gamma=1$ and $p=1$ gives the Fisher equation (1.4).

In recent of work of Szozda (2014), the change of variables of Feng (2007) was applied to the Fisher equation (1.4) to obtain the travelling wave solution of Ablowitz and Zeppetella (1979) expressed in terms of the Weierstrass elliptic function. It was shown that a special case, where the constant of integration in the solution procedure is zero, leads to a travelling wave solution written in a simple closed form in terms of exponential functions with a speed of $c=\frac{5}{\sqrt{6}}$. This solution is well-known; for example, it had been derived earlier by Yuan et al. (2013) using a different method. Szozda (2014) also applied the change of 
variable of Feng (2007) to the case of $p=2$ where equation (1.1) has a third order nonlinear term

$$
\frac{\partial u}{\partial t}=\frac{\partial^{2} u}{\partial x^{2}}+u-u^{3}
$$

For the special case where the constant of the integration obtained in the solution

procedure is zero, a closed form travelling wave with speed $c=\frac{3}{\sqrt{2}}$ expressed in terms of exponential function was obtained.

Here we repeat the solution procedure of Szozda (2014) starting with the change of variables of Feng (2007), but apply it to the general equation (2.7) and obtain an exact solution in closed form for the special case where the constant of the integration is zero. Setting $p=1$ or $p=2$ with $\beta=1$ and $\gamma=1$ gives the two special cases considered by Szozda (2014). If the advection term $\bar{c} \frac{\partial u}{\partial x}$ is included in equation (2.7), the solution is the same as what we obtain here but with $c$ changed by an increment of $\bar{c}$.

\subsection{Derivation of an Exact Solution}

\subsubsection{Exact solution for the case $\gamma \neq 0$}

We first consider the case of $\gamma \neq 0$ in equation (2.7). Introducing the new variables $\xi=x-c t$ and $U(\xi)=u(x, t)$ gives the nonlinear ODE

$$
\frac{d^{2} U}{d \xi^{2}}+c \frac{d U}{d \xi}+\gamma U-\beta U^{p+1}=0 .
$$

Following Feng (2007), we then introduce a new variable $\tau$ given by

$$
\xi=-\frac{1}{c} \ln \tau
$$

which gives

$$
c^{2} \tau^{2} \frac{\mathrm{d}^{2} U}{\mathrm{~d} \tau^{2}}+\gamma U-\beta U^{p+1}=0,
$$

followed by the introduction of new variables $q$ and $\rho(q)$ given by

$$
q=\tau^{m}, \quad U=\tau^{-\frac{1}{2}(m-1)} \rho(q),
$$


where $m$ is a positive real number. This gives

$$
m^{2} c^{2} \tau^{\frac{3 m+1}{2}} \frac{d^{2} \rho}{d q^{2}}+\frac{\left(m^{2}-1\right)}{4} c^{2} \tau^{\frac{-m+1}{2}} \rho+\gamma \tau^{\frac{-m+1}{2}} \rho-\beta \tau^{\frac{-m+1}{2}(p+1)} \rho^{p+1}=0 .
$$

We choose a special choice of $m$ for which the second and third terms cancel each other out in (3.6)

$$
m^{2}=1-\frac{4 \gamma}{c^{2}}
$$

With $|c|>2 \sqrt{\gamma}$ and $\gamma>0$, we have $0<1-\frac{4 \gamma}{c^{2}}<1$ and $m$ is real as we required. We choose the positive square root $m=+\sqrt{1-\frac{4 \gamma}{c^{2}}}$. As shown in section $2.2,|c| \geq 2 \sqrt{\gamma}$ is the condition to obtain a travelling wave solution.

With the choice (3.7), equation (3.6) can be written in terms of $\rho$ and $q$ only as

$$
\frac{d^{2} \rho}{d q^{2}}-\frac{\beta}{m^{2} c^{2}} q^{-m\left(\frac{p+4}{2}\right)+\frac{p}{2}} \rho^{p+1}=0 .
$$

We examine the special case where the exponent of $q$ is zero in the second terms of (3.8). In this case, an exact solution in closed form can be derived. The exponent of $q$ is zero if

$$
m=\frac{p}{p+4}
$$

Since $p$ is real and positive, this choice also implies that $m$ is real and positive as well as required and thus $c>2 \sqrt{\gamma}$ and a travelling wave solution exists. The corresponding value for $c$ is then obtained by substituting this value of $m$ (3.9) into (3.7). This gives

$$
c^{2}=\frac{(p+4)^{2} \gamma}{(2 p+4)}
$$

We then substitute the expressions (3.9) and (3.10) into (3.8) and obtain

$$
\frac{d^{2} \rho}{d q^{2}}=\frac{2 \beta(p+2)}{\gamma p^{2}} \rho^{p+1} .
$$

In order to solve this, we treat the first derivative $\frac{d \rho}{d q}=\rho^{\prime}$ as a function of $\rho$ by writing $\rho^{\prime}=f(\rho)$. In that case, $\rho^{\prime \prime}(q)=f(\rho) f^{\prime}(\rho)$ and equation (3.11) can be written as 


$$
\begin{gathered}
f f^{\prime}=\frac{2(p+2)}{p^{2}} \frac{\beta}{\gamma} \rho^{p+1}, \\
\int f d f=\frac{2(p+2)}{p^{2}} \frac{\beta}{\gamma} \int \rho^{p+1} d \rho, \\
\frac{f^{2}}{2}=\frac{2}{p^{2}} \frac{\beta}{\gamma} \rho^{p+2}-\frac{a}{2}
\end{gathered}
$$

where $a$ is a constant. This gives

$$
\left(\rho^{\prime}\right)^{2}=\frac{4}{p^{2}} \frac{\beta}{\gamma} \rho^{p+2}-a
$$

For the special case where $p=\gamma=\beta=1$ in equation (2.7), the solution of (3.12) can be expressed in terms of the Weierstrass $\wp$ function. The Weierstrass $\wp$ function $\wp\left(z ; g_{2} ; g_{3}\right)$, as described by Abramowitz and Stegun (1964), is the solution of the equation

$$
\left[\wp^{\prime}(z)\right]^{2}=4[\wp(z)]^{3}-g_{2} \wp(z)-g_{3} .
$$

In (3.12), $g_{3}=a$ and $g_{2}=0$. So the solution of (3.12) is $\wp(q-k ; 0 ; a)$ where $k$ is a constant and the solution of the Fisher equation (1.4) is

$$
u(x, t)=e^{\frac{-2}{\sqrt{6}}(x-c t)} \wp\left(\left(e^{\frac{-1}{\sqrt{6}}(x-c t)}\right)-k ; 0 ; a\right),
$$

where $c^{2}=\frac{25}{6}$. This solution was obtained by Ablowitz and Zeppetella (1979) and Szozda (2014) rederived it using the transformation of Feng (2007), as we have done here.

For general $p, \gamma$ and $\beta$, a closed form solution can be obtained for the special case where $a=0$ in (3.12). In that case, equation (3.12) becomes

$$
\begin{gathered}
\rho^{\prime}= \pm \frac{2}{p} \sqrt{\frac{\beta}{\gamma}} \rho^{\frac{p+2}{2}}, \\
\int \rho^{\frac{-p-2}{2}} d \rho= \pm \frac{2}{p} \sqrt{\frac{\beta}{\gamma}} \int d q, \\
\rho=\frac{1}{\left(\mp \sqrt{\frac{\beta}{\gamma}} q+b\right)^{\frac{2}{p}}},
\end{gathered}
$$

where $b$ is a constant. From this, we can express $u$ in terms of $x$ and $t$ as

$$
u(x, t)=\frac{e^{\frac{-1}{2} c(m-1)(x-c t)}}{\left( \pm \sqrt{\frac{\beta}{\gamma}} e^{\frac{-1}{2} c m(x-c t)}+b\right)^{\frac{2}{p}}},
$$


where $c=\frac{ \pm(p+4) \sqrt{\gamma}}{\sqrt{(2 p+4)}}$, as given in $(3.10)$,

$$
u(x, t)=\frac{e^{\frac{-(\operatorname{sgn} c) 2 \sqrt{\gamma}}{\sqrt{2(p+2)}}(x-c t)}}{\left( \pm \sqrt{\frac{\beta}{\gamma}} e^{\frac{-(\operatorname{sgn} c) p \sqrt{\gamma}}{\sqrt{2(p+2)}}(x-c t)}+b\right)^{\frac{2}{p}}} .
$$

If $b>0$ and we choose the negative sign in the denominator $\left(-\sqrt{\frac{\beta}{\gamma}}\right)$, then the expression in (3.16) is singular at the point $\xi$ where $e^{\frac{-c m}{2}(\xi)}=b \sqrt{\frac{\gamma}{\beta}}$. If $b<0$ and we choose the positive sign in denominator $\left(\sqrt{\frac{\beta}{\gamma}}\right)$, then the expression in $(3.16)$ is singular at the point where the exponential $e^{\frac{-c m}{2}(x-c t)}=-b \sqrt{\frac{\gamma}{\beta}}$.

If $p$ is an even integer, then there are two solutions one negative and one positive, because of the power of $\frac{2}{p}$ in the denominator. If $p$ is not an even integer, then there is one positive solution. If $c>0$, then $u \longrightarrow 0$ as $\xi \longrightarrow \infty$ and $u \longrightarrow\left(\frac{\gamma}{\beta}\right)^{\frac{1}{p}}$ as $\xi \longrightarrow-\infty$. If $c<0$, then $u(x, t) \longrightarrow\left(\frac{\gamma}{\beta}\right)^{\frac{1}{p}}$ as $\xi \longrightarrow \infty$ and $u \longrightarrow 0$ as $\xi \longrightarrow-\infty$. That mean for $t>0$ and $c<0 \lim _{x \rightarrow \infty} u(x, t)=$ 0 and $\lim _{x \longrightarrow-\infty} u(x, t)=\left(\frac{\gamma}{\beta}\right)^{\frac{1}{p}}$ and if $c<0$ we have $\lim _{x \longrightarrow \infty} u(x, t)=\left(\frac{\gamma}{\beta}\right)^{\frac{1}{p}}$ and $\lim _{x \rightarrow-\infty} u(x, t)=0$. For the Fisher equation (3.2) with $p=\beta=\gamma=1$, For the singular case, we have positive on one side of the singular point and negative on the other side. Depending on the value of $p$, we can have $u \rightarrow+\infty$ on one side and $u \rightarrow-\infty$ on the other side or $u \rightarrow+\infty$ on both sides or $u \rightarrow \infty$ on one side and no real solution on the other side.

Singular expressions of this type are shown in Figures 3.1 and 3.2. Figure 3.1 shows the case $p=\gamma=\beta=1$ and $c= \pm \frac{5}{\sqrt{6}}$. The red (thick) curve corresponds to $c=\frac{5}{\sqrt{6}}$ and the blue (thin) curve corresponds to $c=-\frac{5}{\sqrt{6}}$. Figure 3.2 shows the case $p=2, \gamma=\beta=1$ and $c= \pm \frac{3}{\sqrt{2}}$. The red line and dotted (thick) curves correspond to $c=\frac{3}{\sqrt{2}}$ and the blue line and dotted (thin) curves $c=-\frac{3}{\sqrt{2}}$.

Theses correspond to trajectories in the $y_{1}, y_{2}$-plane in Figures 2.5 (a) and (d) and 2.6 (a) that extend to infinity as $\xi \rightarrow \infty$ or as $\xi \rightarrow-\infty$

In order to obtain a nonsingular travelling wave solution, we must choose the constant $b$ in the denominator of (3.16) to have the same sign as the square root 


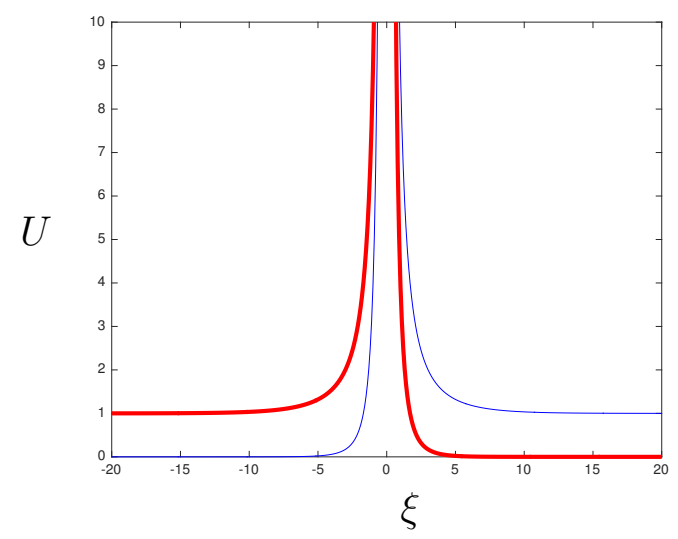

Figure 3.1: The graph of the singular function (3.16) for the case where $b$ has the opposite sign of the square root in the denominator. The parameter values are $p=1$ and $\beta=\gamma=1$. The red (thick) solid curve corresponds to $c>0$. The blue (thin) curve corresponds to $c<0$.

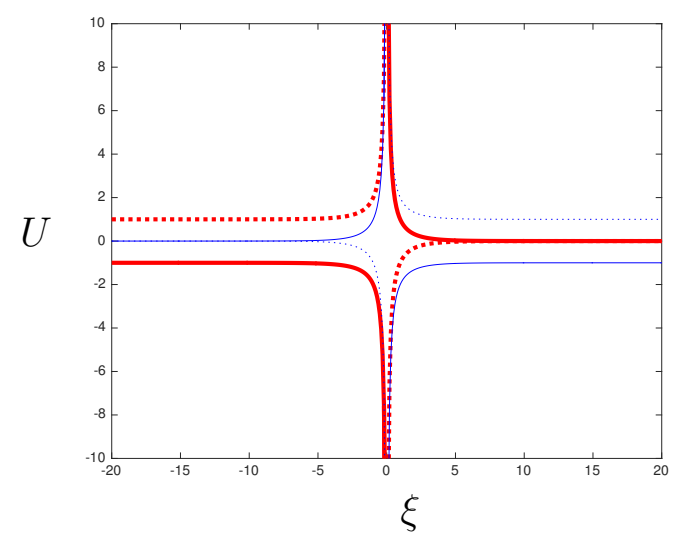

Figure 3.2: The graph of the singular function (3.16) for the case where $b$ has the opposite sign the square root in the denominator. The parameter values are $p=2$ and $\beta=\gamma=1$. The red (thick) curve and the red (thick) dotted curves correspond to $c>0$ and the blue (thin) solid curve. The blue (thin) dotted curves correspond to $c<0$. 
in the denominator. If $p$ is a multiple of 4 , then we must choose the positive square root with $b>0$. In that case, there are two real solutions, one positive and one negative. If $p$ even is not a multiple of 4 , then the positive square root with $b>0$ gives a positive solution and the negative square root with $b<0$ gives a negative solution. If $p$ is an odd or $p=\frac{1}{n}$ where $n$ is integer, we can choose either the positive square root with $b>0$ or the negative square root with $b<0$ and the solution will be positive in both cases. If $p$ is $\frac{1}{n}$ where $n$ is not integer, then we must choose the positive square root with $b>0$. In that case, there is one real positive solution. For the positive solution, for $c>0, u \rightarrow 0$ as $\xi \rightarrow \infty$ and $u \rightarrow\left(\frac{\gamma}{\beta}\right)^{\frac{1}{p}}$ as $\xi \rightarrow-\infty$. Figure 3.3 shows the solution for the case $p=\gamma=\beta=b=1$ and $c= \pm \frac{5}{\sqrt{6}}$. The red (thick) curve corresponds to $c=\frac{5}{\sqrt{6}}$ and the blue (thin) $c=-\frac{5}{\sqrt{6}}$. Figure 3.4 shows the solution for the case $p=2$, $\gamma=\beta=b=1$ and $c= \pm \frac{3}{\sqrt{2}}$. The red (thick) solid curve and the red dotted (thick) curve correspond to $c=\frac{3}{\sqrt{2}}$ and the blue (thin) curve and the blue dotted (thin) curve correspond to $c=-\frac{3}{\sqrt{2}}$

These travelling wave solutions correspond to the heteroclinic orbits that connect the saddle points $(1,0)$ and $(-1,0)$ with the stable node $(0,0)$ in Figures 2.5 (a) and (d) and 2.6 (a).

For the case $c>0$, the slope of the tangent line to the curve at $\xi=0$ is

$$
U_{\xi}(0)=s=\frac{-2 b}{\sqrt{6}(1+b)^{2}} .
$$

An estimate of the width of the wave front (Murray 1989) is

$$
L=\frac{\left(\frac{\gamma}{\beta}\right)^{\frac{1}{p}}}{|s|} .
$$

In the case of the Fisher equation

$$
L=\frac{1}{|s|} \text {. }
$$

In the rest of the thesis we will consider the case with the positive square root in 
the denominator and with $c>0$. In that case (3.16) becomes

$$
u(x, t)=\frac{e^{\frac{-2 \sqrt{\gamma}}{\sqrt{2(p+2)}}(x-c t)}}{\left(\sqrt{\frac{\beta}{\gamma}} e^{\frac{-p \sqrt{\gamma}}{\sqrt{2(p+2)}}(x-c t)}+b\right)^{\frac{2}{p}}}, \quad b>0 .
$$

An initial condition that leads to the travelling wave solution (3.18) is

$$
u(x, 0)=\frac{e^{\frac{-2 \sqrt{\gamma}}{\sqrt{2(p+2)}} x}}{\left(\sqrt{\frac{\beta}{\gamma}} e^{\frac{-p \sqrt{\gamma}}{\sqrt{2(p+2)}} x}+b\right)^{\frac{2}{p}}}, \quad b>0 .
$$

If we consider the advection-diffusion-reaction equation (2.7), with $\bar{c} \neq 0$, then we obtain a solution that is of the form (3.8) with speed $c$ is given by $c=\bar{c}+\frac{(p+4)}{\sqrt{2 p+4}}$. The solution (3.16) becomes

$$
u(x, t)=\frac{e^{\frac{-(\operatorname{sgn}(c-\bar{c}) 2 \sqrt{\gamma}}{\sqrt{2(p+2)}}(x-c t)}}{\left( \pm \sqrt{\frac{\beta}{\gamma}} e^{\frac{-(\operatorname{sgn}(c-\bar{c}) p \sqrt{\gamma}}{\sqrt{2(p+2)}}(x-c t)}+b\right)^{\frac{2}{p}}}, \quad b>0 .
$$

For example, for the case with $p=1, \gamma=\beta=1$ and $c-\bar{c}>0$, the wave speed is $c=\bar{c}+\frac{5}{\sqrt{6}}$. The function (3.18) is the solution of the nondimensional equation (2.7). The solution $u^{*}$ of the dimensional equation (2.1) can be found by substituting (2.5) into (3.18). This gives

$$
u^{*}\left(x^{*}, t^{*}\right)=\frac{e^{\frac{-2 \sqrt{\gamma^{*}}}{\sqrt{6 \nu^{*}}}\left(x^{*}-c^{*} t^{*}\right)}}{\left(\sqrt{\frac{\beta^{*}}{\gamma^{*}}} \frac{-\sqrt{\gamma^{*}}}{\sqrt{6 \nu^{*}}}\left(x^{*}-c^{*} t^{*}\right)+b\right)^{2}} .
$$

\subsubsection{Exact Solution for the case $\gamma=0$}

In this subsection, we consider equation (2.7) with $\gamma=0$,

$$
\frac{\partial u}{\partial t}+\bar{c} \frac{\partial u}{\partial x}=\frac{\partial^{2} u}{\partial x^{2}}-\beta u^{p+1}, \quad p>0 .
$$

If $\bar{c}=0$, then the change of variables $\xi=x-c t$ and $U(\xi)=u(x, t)$ gives

$$
\frac{d^{2} U}{d \xi^{2}}+c \frac{d U}{d \xi}-\beta U^{p+1}
$$






Figure 3.3: Travelling wave solution (3.16) for the case where $b$ has the same sign as the square root in the denominator. The parameter values are $p=1$ and $\beta=\gamma=b=1$. The red (thick) curve corresponds to $c>0$ and the blue (thin) curve corresponds to $c<0$.

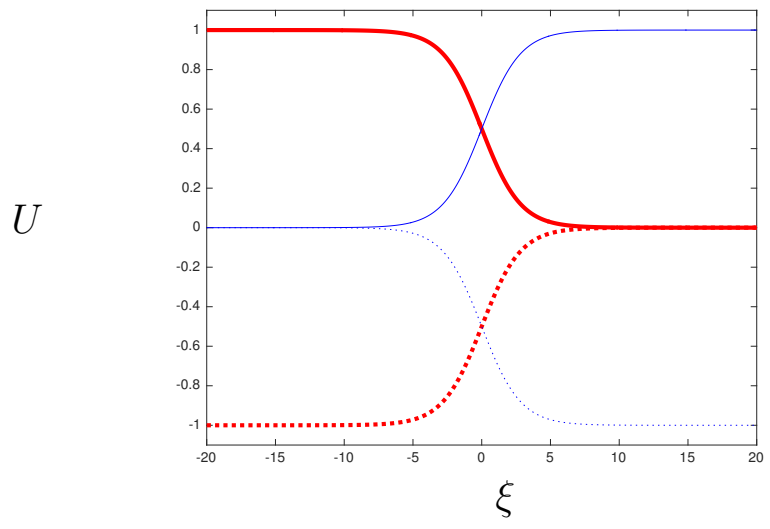

Figure 3.4: Travelling wave solution (3.16) for the case where $b$ has the same sign as the square root in the denominator. The parameter values are $p=2$ and $\beta=\gamma=b=1$. The red (thick) curve and dotted (thick) curves correspond to $c>0$ and the blue (thin) curve and dotted (thin) curves correspond to $c<0$. 
and the change of variable (3.3) gives

$$
\frac{d^{2} U}{d \tau^{2}}-\frac{\beta}{c^{2}} \tau^{-2} U^{p+1}=0
$$

The choice of $p=1$ gives the case considered by Wilhelmsson (1988). They showed that the substitution $u=t^{-1} \phi(z)$ with $z=x t^{-\frac{1}{2}}$ gives

$$
\phi_{z z}=\frac{z}{2} \phi_{z}+\phi-\phi^{2}=0
$$

This equation has a particular solution $\phi=\frac{a_{1}}{a_{3}+z^{2}}+\frac{a_{3}}{\left(a_{3}+z^{2}\right)^{2}}$ where $a_{1}=12(4+\sqrt{6})$, $a_{2}=-24 a_{3}$ and $a_{3}=2(15+5 \sqrt{6})$. For the general case when $p \neq 1$, we do not have a closed form solution.

If $\bar{c} \neq 0$ then the change of variables $\xi=x-\bar{c} t$ and $U(\xi)=u(x-\bar{c} t)$ gives

$$
\frac{d^{2} U}{d \xi^{2}}-\beta U^{p+1}=0
$$

which is of the same form as (3.11). We can solve this by treating the first derivative $\frac{d U}{d \xi}$ as a function of $U$ by writing $U^{\prime}=f(U)$. In this case $U^{\prime \prime}=$ $f(U) f^{\prime}(U)$ and equation (3.23) can be written as

$$
\begin{gathered}
f f^{\prime}=\beta U^{p+1}, \\
\int f d f=\beta \int U^{p+1} d U, \\
f^{2}=\frac{2 \beta}{p+2} U^{p+2}-a,
\end{gathered}
$$

where $a$ is constant. This gives

$$
\left(U^{\prime}\right)^{2}=\frac{2 \beta}{p+2} U^{p+2}-a .
$$

For the special case where $p=1$ and $\beta=6$ in the solution (3.24) can be expressed in terms of the Weierstrauss $\wp$-function which satisfies (3.13).

In (3.24), $g_{3}=a$ and $g_{2}=0$. So the solution of $(3.24)$ is $\wp(\xi-k ; 0 ; a)$ where is $k$ is a constant and the solution of the equation (3.23) is

$$
u(x, t)=\wp((x-c t-k ; 0 ; a) .
$$


If $a=0$ in equation (3.24), then the solution is

$$
u(x, t)=U(\xi)=\frac{1}{\left( \pm \sqrt{\frac{2 \beta}{p+2}} \xi+b\right)^{\frac{2}{p}}}=\frac{1}{\left( \pm \sqrt{\frac{2 \beta}{p+2}}(x-\bar{c} t)+b\right)^{\frac{2}{p}}},
$$

which is singular at the point $\xi= \pm b \sqrt{\frac{p+2}{2 \beta}}$. We have the same possibilities as in the singular case in section 3.2.1. The solution is positive on one side of the singular point and negative on the other side. Depending on the value of $p$ we have $u \rightarrow+\infty$ on one side and $u \rightarrow-\infty$ on the other side or $u \rightarrow+\infty$ on both sides or $u \rightarrow \infty$ on one side and no real solution on the other side. Figures 3.5 and 3.6 show the singular function (3.26) with $p=1$ and $p=2$. 


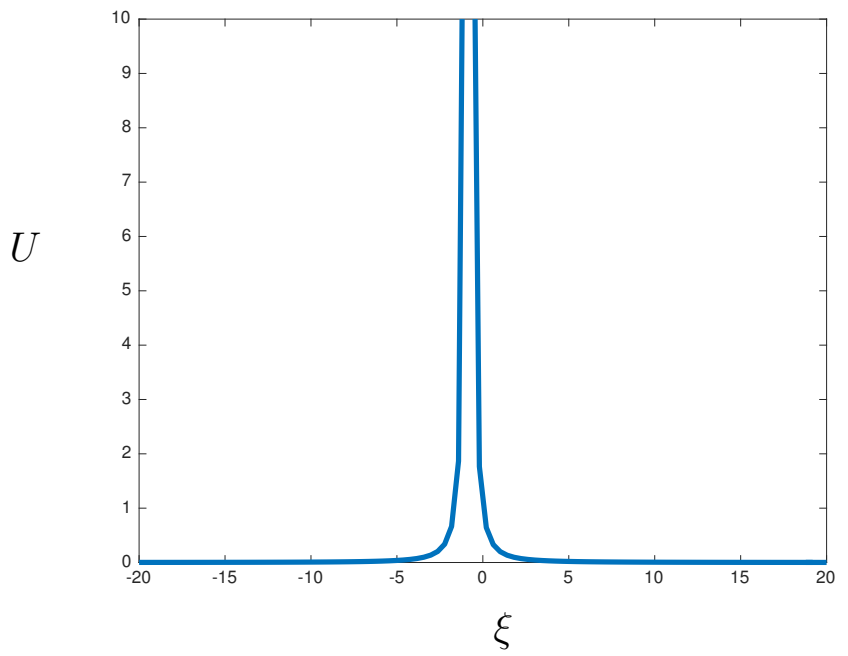

Figure 3.5: The solution (3.26) for the equation (3.22) with $p=1, \bar{c} \neq 0, \gamma=0$, $\beta=1$ and $b=1$ and the positive square root $\sqrt{\frac{2 \beta}{p+2}}$ taken in the denominator of (3.26). This case corresponding to phase portraits in Figure 2.7 if the initial condition to the right of $(1,0)$.



Figure 3.6: The solution (3.26) for the equation (3.22) with $p=2, \bar{c} \neq 0, \gamma=0$, $\beta=1$ and $b=1$ and the positive square root $\sqrt{\frac{2 \beta}{p+2}}$ taken in the denominator of (3.26). 


\section{Chapter 4}

\section{Perturbations to the Travelling Wave Solutions}

\subsection{Introduction}

In chapter 3 we presented derivations of some travelling wave solutions of the nonlinear reaction-diffusion equation (2.7). In certain special cases the solution is obtained in a simple closed form in terms of the Weierstrass $\wp$-function (3.13) and in terms of exponential functions (3.16). The solution (3.16) is in the form of a wave travelling to the right if $c>0$ or to the left if $c<0$. In the case of the Fisher equation(1.4) with $\bar{c} \neq 0$, the wave with $c-\bar{c}>0$ has speed $c=\bar{c}+\frac{5}{\sqrt{6}}$ and an amplitude that approaches 1 at the left (behind the wave front) and approaches 0 at the right (in front of the wave front). Solutions of this type are well-known and their stability properties have been discussed in a number of texts such as Murray (1989). The conditions necessary for the existence of a travelling wave solution were given by Kolmogorov et al. (1937) and generalized by Fife and McLeod (1977) and Bramson (1983, 1986).

In this chapter we state the theorem as presented by Bramson (1983), then we examine some possible sources that can give rise to a perturbation to the 
travelling wave solution. We first consider a configuration where a perturbation is introduced in the initial state and then a configuration where the advection speed $\bar{c}$ of the background medium is perturbed. In each case, we are able to derive an approximate asymptotic expression for the perturbation that arises in the solution. We consider the special case where $\gamma=1, \beta=1, p=1$ and $\bar{c} \neq 0$ in equation (2.6) which is the Fisher equation with an advection term,

$$
\frac{\partial u}{\partial t}+\bar{c} \frac{\partial u}{\partial x}=\frac{\partial^{2} u}{\partial x^{2}}+u(1-u) .
$$

In section 4.1 a spatially varying perturbation is added to the initial condition and in section 4.2 a perturbation that depends on time and space is added to the constant background flow speed $\bar{c}$.

\subsection{Convergence Theorem}

In this section we state the theorem of Bramson (1983) written in the wording of our configuration. We define a travelling wave to be a wave function that travels with time in such a way that the shape of the solution is the same for all time and the speed of propagation of this shape is constant (Murray, 1989).

For a solution $u(x, t)$ of the Fisher equation, Bramson defines a function $m(t)$ by

$$
m(t)=\max \left\{x \in \Re: e^{t} \int_{-\infty}^{\infty} u(y, 0) \frac{e^{-\frac{(x-y)^{2}}{2 t}}}{\sqrt{2 \pi t}} d y \geq 1\right\}
$$

The integral in this expression is the solution of the heat equation

$$
\frac{\partial v}{\partial t}=\frac{1}{2} \frac{\partial^{2} v}{\partial x^{2}}
$$

subject to the initial condition

$$
v(x, 0)=u(x, 0)=f(x) .
$$

The solution of this initial value problem could be obtained, for example, by taking a Fourier transform and solving the resulting equation by making use of 
the Fourier convolution theorem. As an example, if $u(x, t)$ is the function (3.20), then $u(x, 0)$ is the function shown in the red curve in Figure 3.3. Using this as an initial condition for the heat equation (4.2) gives $v(x, t)=\int_{-\infty}^{\infty} u(y, 0) \frac{e^{-\frac{(x-y)^{2}}{4 \nu t}}}{\sqrt{4 \pi \nu t}} d y$ with $\nu=\frac{1}{2}$. The function $\frac{e^{-\frac{x^{2}}{2 t}}}{\sqrt{2 \pi t}}$ is the heat kernel. As $x \rightarrow \infty, u(x, 0) \rightarrow 0$ and for some value of $x$, say $x=x_{2}$, the function is close to zero for $x>x_{2}$. The value $m$ gives a way of defining this number. For $x>m, u(x, 0)$ is so small that the integral $v(x, t)$ becomes smaller then $e^{-t}$. For a function $u(x, t)$ that changes with $t$, the location of $m$ changes with $t$.

The theorem concerns situation where the initial function $u(x, 0)=f(x)$ satisfies $0 \leq f(x) \leq 1$ and $f(x)$ is piecewise continuous (Fife and McLeod, 1977). Theorem 4.1 Bramson (1983)

Consider the nonlinear diffusion-reaction equation

$$
\frac{\partial u}{\partial t}=\frac{1}{2} \frac{\partial^{2} u}{\partial x^{2}}+F(u)
$$

where $F$ is a nonlinear function satisfying

$$
\begin{aligned}
& F(0)=F(1)=0, \quad F(u)>0 \text { for } \quad 0<u<1, \\
& F^{\prime}(u) \leq 1,0<u \leq 1,1-F^{\prime}(u)=O\left(u^{n}\right) \text { as } u \rightarrow \infty, \text { where } n>0 .
\end{aligned}
$$

Let $u(x, t)$ be a solution of (4.4) and suppose that for some $h>0$

$$
\lim _{t \longrightarrow \infty} \frac{1}{t} \log \left(\int_{t}^{t(1+h)} u(y, 0) d y\right)=B,
$$

where $0<|B|<\sqrt{2}$ and for some $\eta, M, N>0$,

$$
\int_{x}^{x+N} u(y, 0) d y>\eta \quad \text { for } x \leq-M .
$$

Then

$$
u(x+m(t), t) \rightarrow W^{\kappa}(x)
$$

uniformly in $x$ as $t \rightarrow \infty$, where $\kappa=c=-\frac{1}{B}-\frac{B}{2}$, or equivalently $B=-c+$ $\sqrt{c^{2}-2}$. 
The proof of this theorem is given by Bramson (1983). The theorem says that if $u(x, t)$ is a function satisfying conditions (4.4)-(4.7) then as $t \rightarrow \infty, u(x+$ $m(t), t)$ becomes a travelling wave $W^{\kappa}(x)$. In the theorem the travelling wave denoted as $W^{\kappa}$ is defined such that $e^{-B x} W^{\kappa}(x) \rightarrow 1$, as $x \rightarrow \infty$ for some $B<0$.

Equation (4.4) corresponds to the dimensional equation (1.1) with diffusion coefficient $\nu=\frac{1}{2}$. In our case, we nondimensionalized (1.1) so that the diffusion coefficient became $\nu=1$. With $F(u)=u(1-u)$ we obtained the solution

$$
u(x, t)=\frac{e^{\frac{-2}{\sqrt{6}}(x-c t)}}{\left(e^{\frac{-1}{\sqrt{6}}(x-c t)}+b\right)^{2}},
$$

where $c=\frac{5}{\sqrt{6}}$ and $b>0$. For general $\nu$, the theorem holds with the same conditions (4.5), (4.6) and (4.7), with $B$ depending on $\nu$ and $0<|B|<\sqrt{2}$, but in the condition (4.8) we would instead have $\kappa=-B \nu-\frac{1}{B}$. So for $\nu=1$, we have $\kappa=c=-B-\frac{1}{B}$ or equivalently $B=\frac{-c+\sqrt{c^{2}-4}}{2}$ the eigenvalue expression in (2.16) with $\gamma=1$. For general $\nu, m$ is defined as

$$
m(t)=\max \left\{x \in \Re: e^{t} \int_{-\infty}^{\infty} u(y, 0) \frac{e^{-\frac{(x-y)^{2}}{2 \nu t}}}{\sqrt{2 \pi \nu t}} d y \geq 1\right\}
$$

\subsection{Perturbation to the Initial Condition}

The exact solutions obtained in the special cases in chapter 3 correspond to some specific initial conditions. With other more general initial conditions, while it may not be possible to obtain exact solution in closed form, it would be desirable to obtain information about the qualitative behaviour of the solution for finite time and for infinite time.

In this section we perturb the travelling wave solution of the Fisher equation (4.1) by adding an $x$-dependent perturbation to the initial condition and we seek to obtain an expression for the resulting perturbation to the solution. We consider the initial-value problem comprising equation (4.1) subject to an initial condition 
of the form

$$
u(x, 0)=f(x)=f^{(0)}(x)+\varepsilon f^{(1)}(x),
$$

where

$$
f^{(0)}(x)=\frac{e^{-\frac{2}{\sqrt{6}} x}}{\left(e^{-\frac{1}{\sqrt{6}} x}+b\right)^{2}}
$$

and $f^{(1)}(x)$ is a specified function that satisfies the limiting conditions $f^{(1)} \rightarrow 0$ as $x \rightarrow \pm \infty$.

The perturbation $\varepsilon f^{(1)}$ added to the initial condition gives rise to an $O(\varepsilon)$ perturbation to the solution and the solution can then be written as an expansion in powers of $\varepsilon$,

$$
u(x, t) \sim u^{(0)}(x, t)+\varepsilon u^{(1)}(x, t), \quad \varepsilon \rightarrow 0,
$$

and substitute this into equation (4.1) to obtain

$$
\begin{aligned}
-c \frac{\partial u^{(0)}}{\partial t} & +\bar{c} \frac{\partial u^{(0)}}{\partial x}+\varepsilon\left(\frac{\partial u^{(1)}}{\partial t}+\bar{c} \frac{\partial u^{(1)}}{\partial x}\right) \\
& =\frac{\partial^{2} u^{(0)}}{\partial x^{2}}+u^{(0)}\left(1-u^{(0)}\right)+\varepsilon\left(\frac{\partial^{2} u^{(1)}}{\partial x^{2}}-2 u^{(0)} u^{(1)}+u^{(1)}\right)+O\left(\varepsilon^{2}\right) .
\end{aligned}
$$

The leading order term $u^{(0)}(x, t)$ satisfies the Fisher equation (1.4) with the initial condition $u^{(0)}(x, 0)=f^{(0)}(x)$ where $f^{(0)}(x)$ is given by (4.11). This means that

$$
u^{(0)}(x, t)=U^{(0)}(x-c t)=U^{(0)}(\xi)
$$

where $U^{(0)}$ satisfies

$$
-(c-\bar{c}) \frac{d U^{(0)}}{d \xi}=\frac{d^{2} U^{(0)}}{d \xi^{2}}+U^{(0)}\left(1-U^{(0)}\right),
$$

and thus

$$
u^{(0)}(x, t)=U^{(0)}(\xi)=f^{(0)}(\xi) .
$$

At $O(\varepsilon)$ we obtain

$$
\frac{\partial u^{(1)}}{\partial t}+\bar{c} \frac{\partial u^{(1)}}{\partial x}=\frac{\partial^{2} u^{(1)}}{\partial x^{2}}+\left(1-2 U^{(0)}\right) u^{(1)}
$$


with initial condition $u^{(1)}(x, 0)=f^{(1)}(x)$. The form of this equation suggests that $u^{(1)}$ depends on $\xi=x-c t$. More generally, $u^{(1)}$ may depend explicitly on $x$ and $t$ as well as $\xi$ and this is examined in section 4.3.2.

If the initial function $f(x)=f^{(0)}(x)+\varepsilon f^{(1)}(x)$ satisfies conditions (4.6) and (4.7) then the solution $u$ converge to a travelling wave solution as $t \rightarrow \infty$ according to Theorem 4.1. To determine the conditions on $f^{(1)}$ for the conclusions of the theorem to hold we consider the integral

$$
\int_{t}^{t+t h}\left(f^{(0)}(y)+\varepsilon f^{(1)}(y)\right) d y
$$

and note that $f^{(0)}(y) \sim \frac{e^{-\frac{2}{\sqrt{6}} y}}{b^{2}}$ as $y \rightarrow \infty$. That means that

$$
\begin{gathered}
\int_{t}^{t+t h}\left(f^{(0)}(y)\right) d y \sim \frac{\sqrt{6} e^{-\frac{2}{\sqrt{6}} t}}{2 b^{2}}\left(1-e^{-\frac{2}{\sqrt{6}} t h}\right) \\
\sim \frac{\sqrt{6} e^{-\frac{2}{\sqrt{6}} t}}{2 b^{2}}, \quad t \rightarrow \infty .
\end{gathered}
$$

Taking the logarithm of this, dividing by $t$ and then taking the limit as $t \rightarrow \infty$ gives the limit $B=-\frac{2}{\sqrt{6}}$ in the theorem. If

$$
f^{(1)}(x) \ll e^{-\frac{2}{\sqrt{6}} x}, \quad x \rightarrow \infty,
$$

then $f^{(0)}+\varepsilon f^{(1)}$ satisfies the condition (4.6) with the same value of $B=-\frac{2}{\sqrt{6}}$. We also observe that the condition (4.7) holds for $f^{(0)}$ because we can choose $M, N>0$ such that for $x \leq N-M<0, f^{(0)}$ is close to 1 , i.e., $1-a<f^{(0)}(x)<1$ for some $a>0$. In that case $\int_{x}^{x+N} f^{(0)}(y) d y>N(1-a)$ for $x \leq-M$ and we can choose $\eta$ in condition(4.7) such that $\eta=N(1-a)$. If

$$
f^{(1)}(x) \geq 0 \quad \text { for } \quad x \leq N-M,
$$

then $\int_{x}^{x+N} f^{(0)}(y)+\varepsilon f^{(0)}(y) d y \geq \int_{x}^{x+N} f^{(0)}(y) d y>\eta$. So if $f^{(1)}(y)$ satisfies (4.17), the perturbed solution will converge to a travelling wave. In section 4.3 we consider different forms of $f^{(1)}$, some of which give travelling wave solutions and some of which do not. 


\subsection{1 $\xi$-dependent Perturbation}

We consider equation (4.15) and write $u^{(1)}(x, t)=U^{(1)}(\xi)$. In this case the solution $u^{(1)}$ is a travelling wave because it depends on $\xi$ only. This gives

$$
U_{\xi \xi}^{(1)}+(c-\bar{c}) U_{\xi}^{(1)}+\left[1-2 f^{(0)}(\xi)\right] U^{(1)}=0 .
$$

To eliminate the first derivative term in (4.18), we write

$$
U^{(1)}(\xi)=h(\xi) e^{\frac{-(c-\bar{c})}{2} \xi},
$$

which transforms (4.18) into

$$
h_{\xi \xi}-\left(2 f^{(0)}(\xi)+\frac{(c-\bar{c})^{2}}{4}-1\right) h=0 .
$$

We define

$$
Q(\xi)=2 f^{(0)}(\xi)+\frac{(c-\bar{c})^{2}}{4}-1 .
$$

The function $f^{(0)}(\xi)$ is slowly-varying with respect to $\xi$. Thus, as $\xi \rightarrow \infty$ and $\xi \rightarrow-\infty Q(\xi)$ is slowly varying with respect to $\xi$ as $\xi \rightarrow \infty$ and $\xi \rightarrow-\infty$ and can be written as

$$
Q(\xi)=q(\delta \xi)=q(\tau), \quad\left|\frac{d Q}{d \xi}\right| \sim \delta \quad \text { for some } \delta \ll 1
$$

we can use the WKB method which described in Appendix B.

Since $(c-\bar{c})^{2}=\frac{25}{6}$ and $0<f^{(0)}(\xi)<1$ for all $\xi \in \Re$,

$$
Q(\xi)=2 f^{(0)}(\xi)+\frac{1}{24}>0
$$

for all $\xi \in \Re$.

Thus the WKB approximation for the solution of (4.20) takes the form

$$
h(\xi)=A_{1} h_{1}(\xi)+B_{1} h_{2}(\xi),
$$

where

$$
h_{1} \sim \frac{\exp \left(\int_{a}^{\xi} \sqrt{Q} d \bar{\xi}\right)}{\sqrt[4]{Q}}, \quad h_{2} \sim \frac{\exp \left(-\int_{a}^{\xi} \sqrt{Q} d \bar{\xi}\right)}{\sqrt[4]{Q}}, \quad \text { as } \quad|\xi| \rightarrow \infty
$$


and $A_{1}$ and $B_{1}$ are constants and $a$ is an arbitrary fixed value of $\xi$, and the solution of (4.18) is

$$
u^{(1)}(x, t)=U^{(1)}(\xi) \sim \frac{A_{1} \exp \left(\int_{a}^{\xi} \sqrt{Q} d \bar{\xi}\right)+B_{1} \exp \left(-\int_{a}^{\xi} \sqrt{Q} d \bar{\xi}\right)}{\sqrt[4]{Q}} \exp \left(\frac{-(c-\bar{c}) \xi}{2}\right) .
$$

The form of the function $f^{(0)}(\xi)$ is such that it can be approximated by 1 for $\xi$ less than some value $\xi_{1}$ and and it can be approximated by 0 for $\xi$ greater than some value $\xi_{2}$. Setting $f^{(0)}(\xi)$ to 1 gives

$$
\begin{gathered}
h_{1}(\xi) \sim e^{\left(\frac{7}{2 \sqrt{6}}\right) \xi}, \quad h_{2}(\xi) \sim e^{\left(-\frac{7}{2 \sqrt{6}}\right) \xi}, \\
U^{(1)}(\xi) \sim \sqrt[4]{24}\left(A_{1} e^{\frac{1}{\sqrt{6}} \xi}+B_{1} e^{\frac{-6}{\sqrt{6}} \xi}\right), \quad \xi \rightarrow-\infty
\end{gathered}
$$

In order to have a bounded solution as $\xi \rightarrow-\infty$, we must have $B_{1}=0$. Setting $f^{(0)}(\xi)$ to 0 gives

$$
\begin{gathered}
h_{1}(\xi) \sim e^{\left(\frac{1}{2 \sqrt{6}}\right) \xi}, \quad h_{2}(\xi) \sim e^{\left(-\frac{1}{2 \sqrt{6}}\right) \xi}, \\
U^{(1)}(\xi) \sim \sqrt[4]{24}\left(A_{2} e^{\frac{-2}{\sqrt{6}} \xi}+B_{2} e^{\frac{-3}{\sqrt{6}} \xi}\right), \quad \xi \rightarrow \infty,
\end{gathered}
$$

where $A_{2}$ and $B_{2}$ are constants.

For $\xi_{1}<\xi<\xi_{2}$, the functions $f^{(0)}$ and $Q$ are not slowly varying functions of $\xi$, so neither of the expressions (4.27) and (4.29) can be used as approximations. In the interval $\xi_{1}<\xi<\xi_{2}$, we can find a solution to the O.D.E (4.18) as a power series in powers of $\xi$ by expanding $f^{(0)}$ as a power series and substituting it into (4.18). The first few terms of the power series for $f^{(0)}$ give the approximation

$$
f^{(0)}(\xi) \approx \frac{1}{(1+b)^{2}}-\frac{2 b}{\sqrt{6}(1+b)^{3}} \xi+\frac{b}{6} \frac{(2 b-1)}{(b+1)^{4}} \xi^{2} .
$$

The graph of the approximation (4.30) is shown in Figure 4.1 compared with the exact expression $f^{(0)}(\xi)$. Thus,

$$
1-2 f^{(0)}(\xi)=\sum_{k=0}^{\infty} a_{k} \xi^{k} \approx a_{0}+a_{1} \xi+a_{2} \xi^{2}
$$

where $a_{0}=1-\frac{2}{(1+b)^{2}}, a_{1}=\frac{4}{\sqrt{6}(1+b)^{3}}$ and $a_{2}=\frac{2 b}{3} \frac{(3 b-2)}{(b+1)^{4}}$. 




Figure 4.1: Illustration of an approximation for the function $f^{(0)}(\xi)$. The red (thin) curve shows the function $f^{(0)}(\xi)$ given by (3.18) and the blue (thick) curve shows the approximation (4.30)

We seek a power series solution for $U^{(1)}$ in the form

$$
U^{(1)}(\xi)=\sum_{k=0}^{\infty} C_{k} \xi^{k},
$$

where $C_{k}$ are constants. Substituting (4.31) and (4.32) into (4.18) gives

$$
\begin{aligned}
\left(2 C_{2}+6 C_{3} \xi\right. & \left.+12 C_{4} \xi^{2}+20 C_{5} \xi^{3}+. .\right)+(c-\bar{c})\left(C_{1}+2 C_{2} \xi+3 C_{3} \xi^{2}+4 C_{4} \xi^{3}+. .\right) \\
& +\left(a_{0}+a_{1} \xi+a_{3} \xi^{2}+. .\right)\left(C_{0}+C_{1} \xi+C_{2} \xi^{2}+C_{3} \xi^{3}+. .\right)=0 .
\end{aligned}
$$


We group together terms with like powers of $\xi$ and find

$$
\begin{gathered}
C_{2}=\frac{-(c-\bar{c}) C_{1}-a_{0} C_{0}}{2}, \\
C_{3}=\frac{-2(c-\bar{c}) C_{2}-a_{0} C_{1}-a_{1} C_{0}}{6}, \\
C_{4}=\frac{-3(c-\bar{c}) C_{3}-a_{0} C_{2}-a_{1} C_{1}-a_{2} C_{0}}{12},
\end{gathered}
$$

In general, we can see that

$$
C_{k}=\frac{-(k-1)(c-\bar{c}) C_{k-1}-\sum_{n=0}^{k-2} a_{n} C_{k-2-n}}{k(k-1)},
$$

for $k \geq 2$. We can prove this general formula for $C_{k}$ using mathematical induction.

We observe that each coefficient $C_{k}, k \geq 2$, can be expressed as a linear combination of $C_{0}$ and $C_{1}$. Grouping together all the terms involving $C_{0}$ and grouping together all the terms involving $C_{1}$, the series can be written as

$$
U^{(1)}(\xi)=C_{0} g_{0}(\xi)+C_{1} g_{1}(\xi)
$$

where $g_{0}(\xi)=1+\sum_{n=2}^{k-2} \kappa_{0 n} \xi^{n}$ and $g_{1}(\xi)=\xi+\sum_{n=2}^{k-2} \kappa_{1 n} \xi^{n}$ and $\kappa_{0 n}$ and $\kappa_{1 n}, n=$ $2, . ., k-2$ are constants that depend on $(c-\bar{c})$ and on the coefficients $a_{0}, a_{1}, a_{2}, \ldots$ The constants $C_{0}$ and $C_{1}$ depend on the initial condition specified. To determine the interval of convergence of this series solution, we note (4.18) is a variable coefficient O.D.E with coefficients that are analytic function of $\xi$. A standard theorem from the linear O.D.Es (see, for example, Tenenbaum, 1985) tells us that the radius of convergence of the power series solution (4.32) is at least as great as the distance from $\xi=0$ to the singular point of the equation (4.18) which is closest to $\xi=0$ in the complex $\xi$-plane. Since the equation (4.18) has no singularity, the radius of convergence is infinite. Thus, (4.34) is valid for all $\xi$.

If the solution (4.34) approximated by the first few terms in the series the resulting error of the approximation is $O\left(\xi^{n+1}\right)$ according to Taylor's Remainder 
theorem. Figure 4.2 shows the graph of the approximation $U^{(1)}=\sum_{k=0}^{4} C_{k} \xi^{k}$ are the interval $-4<\xi<4$ for some arbitrary choices of $C_{0}$ and $C_{1}$. For $|\xi|>4$ the graphs of the asymptotic expressions (4.27) and (4.29) are shown for values of the constants $A_{1}, A_{2}, B_{2}$ and $B_{2}$ that allow these expressions to match as $\xi \rightarrow \xi_{2}$ and $\xi \rightarrow \xi_{1}$.

Since $u^{(1)}$ is a function of $\xi$ only, it is a travelling wave with speed $c$ and thus $u(x, t)=u^{(0)}+\varepsilon u^{(1)}$ is a travelling wave.

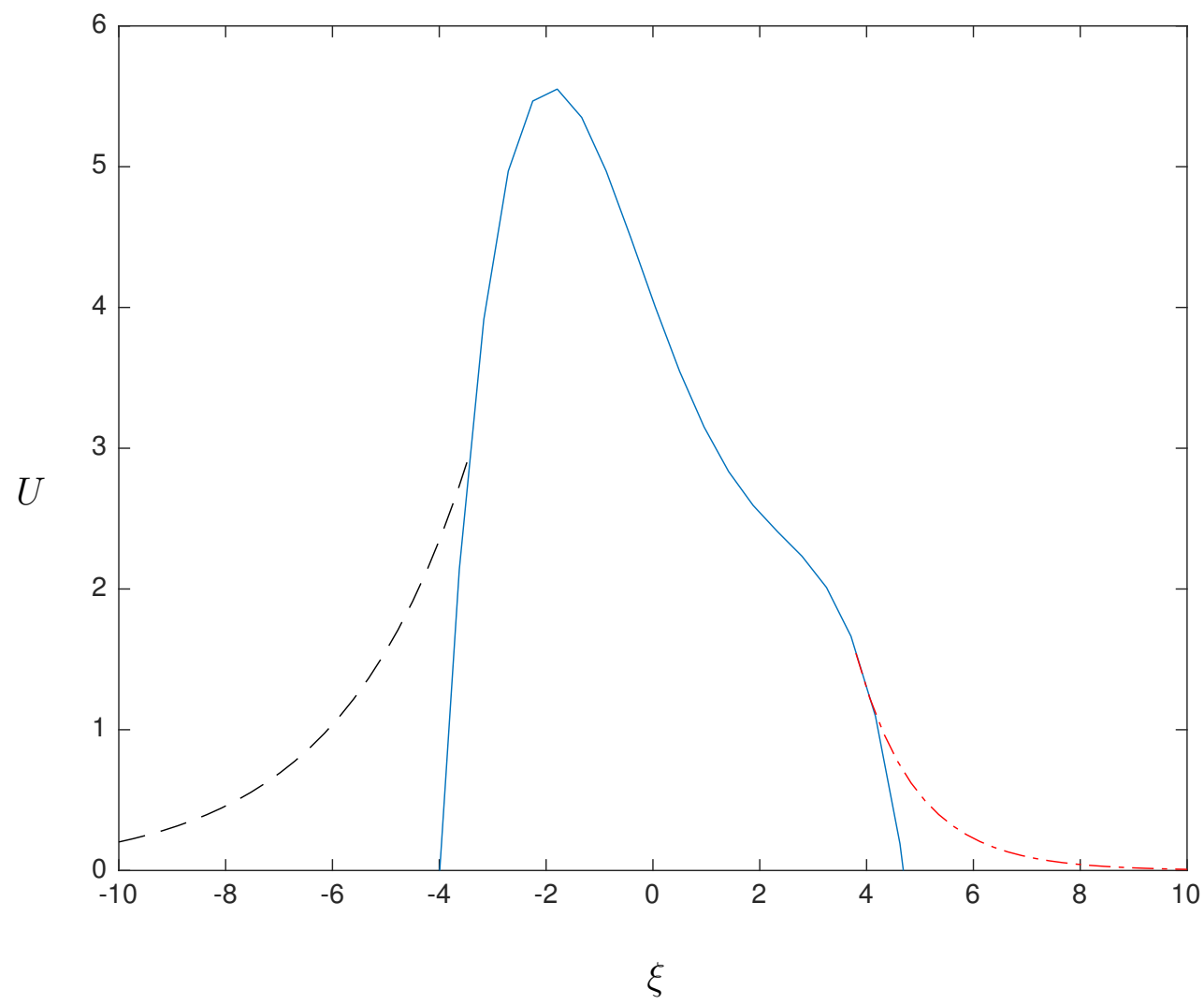

Figure 4.2: Illustration of the form of the asymptotic solution $u^{(1)}(x, t)=U^{(1)}(\xi)$ for $\xi<\xi_{1}$ (the black dashed curve), $\xi_{1}<\xi<\xi_{2}$ (the blue solid curve) and $\xi<\xi_{2}$ (the red dash-dot curve) 


\subsection{2 $\xi$-, $t$ - and $x$-dependent Perturbation}

In section 4.3.1 we found an approximation for the solution $u^{(1)}(x, t)=U^{(1)}(\xi)$ corresponding to the perturbed initial condition (4.11). More generally, $u^{(1)}$ may depend explicitly on $t$ as well as $\xi$, as shown in Murray (1989). In this section we consider a perturbation that depends explicitly on both $t$ and $x$, as well as $\xi$. We consider the case where the solution of equation (4.15) takes the form $u^{(1)}(x, t)=\alpha(\xi) \beta(x, t)$, where $\xi=x-c t$. Substituting this form into (4.15) gives

$$
\begin{aligned}
\alpha_{\xi \xi}(\xi)+(c-\bar{c}) \alpha_{\xi}(\xi)+\alpha(\xi)\left(1-2 U^{(0)}\right) & \\
& =\left[\frac{\beta_{t}+\bar{c} \beta_{x}-\beta_{x x}}{\beta}\right] \alpha(\xi)-\frac{2 \beta_{x}}{\beta} \alpha_{\xi}(\xi)
\end{aligned}
$$

Since the left hand side depends on $\xi$ only, the function $\beta$ must chosen so that the right hand side also depends on $\xi$ only. This leads to the choice $\beta=e^{-\lambda t} e^{-\mu x}$. For $x>0$ then we obtain

$$
\alpha_{\xi \xi}+(c-\bar{c}-2 \mu) \alpha_{\xi}+\left(1+\lambda+\bar{c} \mu+\mu^{2}-2 U^{(0)}\right) \alpha=0 .
$$

To eliminate the first derivative we perform the change of variable

$$
\alpha(\xi)=h(\xi) e^{\frac{-(c-\bar{c}-2 \mu)}{2} \xi},
$$

Substituting this into (4.36) gives

$$
h_{\xi \xi}-Q(\xi) h=0
$$

where

$$
Q(\xi)=2 f^{(0)}(\xi)+\frac{(c-\bar{c}-2 \mu)^{2}}{4}-\mu^{2}-\lambda-\bar{c} \mu-1 .
$$

Simplifying this expression and setting $(c-\bar{c})=\frac{5}{\sqrt{6}}$ gives

$$
Q(\xi)=2 f^{(0)}(\xi)+\frac{1}{24}-(\lambda+c \mu)
$$

or

$$
Q(\xi)=2 f^{(0)}(\xi)+\frac{1}{24}-\sigma
$$


where $\sigma=\lambda+c \mu$.

For $x<0$, we obtain the same expression (4.39) for $Q$ but with $\sigma=\lambda-c \mu$.

For values of $\xi$ for which $Q(\xi)>0$, two linearly independent solutions of equation (4.38) are

$$
h_{1}(\xi) \sim \frac{\exp \left(\int_{a}^{\xi} \sqrt{Q} d \bar{\xi}\right)}{\sqrt[4]{Q}}, \quad h_{2}(\xi) \sim \frac{\exp \left(-\int_{a}^{\xi} \sqrt{Q} d \bar{\xi}\right)}{\sqrt[4]{Q}},|\xi| \quad \text { as } \rightarrow \infty .
$$

For values of $\xi$ for which $Q(\xi)<0$, two linearly independent solutions of equation (4.37) are

$$
h_{1}(\xi) \sim \frac{\cos \left(\int_{a}^{\xi} \sqrt{-Q} d \bar{\xi}\right)}{\sqrt[4]{-Q}} \quad h_{2}(\xi) \sim \frac{\left.\sin \left(\int_{a}^{\xi} \sqrt{-Q} d \bar{\xi}\right)\right]}{\sqrt[4]{-Q}}, \quad \text { as }|\xi| \rightarrow \infty
$$

If $\sigma<\frac{1}{24}$, then $Q(\xi)>0$ for all $\xi$, and so the solution is a linear combination of the exponential functions (4.40). If $\sigma=\frac{1}{24}$, then $Q(\xi)>0$, so the solution is a linear combination of the exponential functions (4.40) for $\xi \rightarrow-\infty$, and $Q(\xi) \rightarrow$ $0^{+}$as $\xi \rightarrow \infty$ and so the solution is asymptotic to a constant for $\xi \rightarrow \infty$. If $\sigma=\frac{49}{24}$, then $Q(\xi)<0$, so the solution is linear combination of the oscillatory functions (4.41) as $\xi \rightarrow \infty$ and $Q(\xi) \rightarrow 0^{-}$as $\xi \rightarrow-\infty$, the solution is asymptotic to a constant. If $\sigma>\frac{49}{24}$ then $Q(\xi)<0$ for all $\xi$ so the solution is linear combination of the oscillatory function (4.41).

If $\frac{49}{24} \geq \sigma>\frac{1}{24}$ then there is a turning point. To find the turning point we solve the equation $Q(\bar{\xi})=0$. This can be written as

$$
\begin{gathered}
2 f^{(0)}(\xi)=1+\lambda+\bar{c} \mu+\mu^{2}-\frac{(c-\bar{c}-2 \mu)^{2}}{4} \\
\frac{e^{\frac{-1}{\sqrt{6}} \xi}}{\left(e^{\frac{-1}{\sqrt{6}} \xi}+b\right)^{2}}=\frac{1}{2}\left(\lambda-\frac{(c-\bar{c}-2 \mu)^{2}}{4}+1\right), \\
\left(1+b e^{\frac{1}{\sqrt{6}} \xi}\right)=\frac{1}{\sqrt{\frac{1}{2}\left(\lambda-\frac{(c-\bar{c}-2 \mu)^{2}}{4}+1\right)}},
\end{gathered}
$$




$$
e^{\frac{1}{\sqrt{6} \xi}}=\frac{1}{b \sqrt{\frac{1}{2}\left(\lambda-\frac{(c-\bar{c}-2 \mu)^{2}}{4}+1\right)}}-\frac{1}{b},
$$

Thus, the turning point is at

$$
\bar{\xi}=\sqrt{6}\left\{\ln \left(\frac{1}{b \sqrt{\frac{1}{2}+\frac{\left(\lambda+\bar{c} \mu+\mu^{2}\right)}{2}-\frac{(c-\bar{c}-2 \mu)^{2}}{8}}}-\frac{1}{b}\right)\right\},
$$

So the solution is a linear combination of the exponential functions (4.40) for $\xi<\xi_{1}$ and a linear combination of the oscillatory functions (4.41) for $\xi>\xi_{2}$. For $\xi_{1}<\xi<\xi_{2}$ an approximation for the solution can be found as a series in powers of $\xi$ as in section 4.3.1. A summary of the different types of solution corresponding to different values of $\sigma$ is given in Table 4.1.

\begin{tabular}{||c|c|c||}
\hline & Solution $h(\xi)$ as $\xi \rightarrow-\infty$ & Solution $h(\xi)$ as $\xi \rightarrow \infty$ \\
$\sigma<\frac{1}{24}$ & exponential & exponential \\
$\sigma 4$ & exponential & approximately constant \\
$\frac{1}{24}<\sigma<\frac{49}{24}$ & exponential & oscillatory \\
$\sigma=\frac{49}{24}$ & approximately constant & oscillatory \\
$\sigma>\frac{49}{24}$ & oscillatory & oscillatory \\
\hline \hline
\end{tabular}

Table 4.1: Summary of the types of solution $h(\xi)$ as $\xi \rightarrow-\infty$ and as $\xi \rightarrow \infty$ corresponding to different values of $\sigma$.

Thus, the solution of $(4.15)$ is

$$
u^{(1)}(x, t)=h(x-c t) \exp \left(\frac{-(c-\bar{c}-2 \mu)}{2} \xi-\mu|x|-\lambda t\right),
$$

where $h$ is given by (4.40) or (4.41) and the initial condition is $u^{(1)}(x, 0)=$ $f^{(1)}(x)=h(x) e^{-\frac{1}{2}(c-\bar{c}) x}$. We note that there is no explicit $x$-dependence in these expressions. However, the value of the constant $\mu$ affects the rate of exponential decay with $t$. 
For $x>0$ the solution $(4.43)$ can be written as

$$
u^{(1)}(x, t)=h(x-c t) \exp \left(-\frac{1}{2}(c-\bar{c}) \xi-(\lambda+c \mu) t\right) .
$$

For the cases where $h_{1}$ and $h_{2}$ are exponential functions we have

$$
h \sim A e^{\frac{1}{2 \sqrt{6}} \xi}+B e^{\frac{-1}{2 \sqrt{6}} \xi}+e^{\left(\frac{1}{2 \sqrt{6}}-\mu\right) \xi}, \quad \xi \rightarrow \infty
$$

which gives

$u^{(1)} \sim\left(A e^{\frac{(x-c t)}{2 \sqrt{6}}}+B e^{\frac{-(x-c t)}{2 \sqrt{6}}}+e^{\left(\frac{1}{2 \sqrt{6}}-\mu\right)(x-c t)}\right) e^{-\frac{5}{2 \sqrt{6}} x} e^{\left(\frac{5}{2 \sqrt{6}} c t-(\lambda+c \mu) t\right)}, \quad(x-c t) \rightarrow \infty$.

For fixed $x$, as $t \rightarrow \infty$, we have

$$
\begin{aligned}
u^{(1)} & \sim\left(A e^{\frac{-c}{2 \sqrt{6}} t}+B e^{\frac{c}{2 \sqrt{6}} t}+e^{-\left(\frac{1}{2 \sqrt{6}}-\mu\right) c t}\right) e^{\left(\frac{5}{2 \sqrt{6}} c t-(\lambda+c \mu) t\right)} \\
& =A e^{\left(\frac{2}{\sqrt{6} c t}-(\lambda+c \mu) t\right)}+B e^{\left(\frac{3}{\sqrt{6}} c t-(\lambda+c \mu) t\right)}+e^{\left(\frac{2}{\sqrt{6}} c-\lambda\right) t} .
\end{aligned}
$$

Considering the dominant term, if $\mu<\frac{1}{\sqrt{6}}$ we see that $u^{(1)} \sim e^{\left(\frac{3}{\sqrt{6}} c t-(\lambda+c \mu) t\right)}$ as $t \rightarrow \infty$. In order to have a bounded solution $u^{(1)}$ as $t \rightarrow \infty$ we require that $\lambda+c \mu \geq \frac{3}{\sqrt{6}} c$. If $\lambda+c \mu=\frac{3}{\sqrt{6}} c$ then $u^{(1)} \sim O(1)$ as $t \rightarrow \infty$. If $\mu>\frac{1}{\sqrt{6}}$ we see that $u^{(1)} \sim e^{\left(\frac{2}{\sqrt{6}} c-\lambda\right) t}$ as $t \rightarrow \infty$. In order to have a bounded solution $u^{(1)} \rightarrow 0$ as $t \rightarrow \infty$ we require that $\lambda \geq \frac{2}{\sqrt{6}} c$ which means $\lambda+c \mu \geq \frac{3}{\sqrt{6}} c$.

For fixed $t$, as $x \rightarrow \infty$, we have

$$
\begin{aligned}
& u^{(1)} \sim\left(A e^{\frac{1}{2 \sqrt{6}} x}+B e^{\frac{-1}{2 \sqrt{6}} x}+e^{\left(\frac{1}{2 \sqrt{6}}-\mu\right) x}\right) e^{\left(-\frac{5}{2 \sqrt{6}}\right) x} \\
& \sim\left(A e^{\frac{-2}{\sqrt{6}} x}+B e^{\frac{-3}{\sqrt{6}} x}+e^{\left(\frac{-2}{\sqrt{6}}-\mu\right) x}\right) .
\end{aligned}
$$

Considering the dominant term, we see that $u^{(1)} \sim e^{\left(-\frac{2}{\sqrt{6}}\right) x}$ as $x \rightarrow \infty$, which means that $u^{(1)} \rightarrow 0$ as $x \rightarrow \infty$. Thus this solution satisfies the condition (4.16) at $t=0$.

For the cases where $h_{1}$ and $h_{2}$ are oscillatory or approximately constant, we have

$$
h \sim O(1)+e^{\left(\frac{1}{2 \sqrt{6}}-\mu\right) \xi}, \quad \xi \rightarrow \infty
$$


which gives

$$
\begin{gathered}
u^{(1)} \sim\left(O(1)+e^{\left(\frac{1}{2 \sqrt{6}}-\mu\right)(x-c t)}\right) e^{-\frac{5}{2 \sqrt{6}} x} e^{\left(\frac{5}{2 \sqrt{6}} c t-(\lambda+c \mu) t\right)}, \quad(x-c t) \rightarrow \infty \\
\sim e^{-\frac{5}{2 \sqrt{6}} x} e^{\left(\frac{5}{2 \sqrt{6}} c t-(\lambda+c \mu) t\right)}+e^{\left(\frac{-2}{\sqrt{6}}-\mu\right) x} e^{\left(\frac{2}{\sqrt{6}} c-\lambda\right) t} .
\end{gathered}
$$

For fixed $x$ as $t \rightarrow \infty$, we have $u^{(1)} \sim e^{\left(\frac{2}{\sqrt{6}} c-\lambda\right) t}$ as $t \rightarrow \infty$. If $\mu<\frac{1}{2 \sqrt{6}}$, then in order to have a bounded solution $u^{(1)}$ as $t \rightarrow \infty$ require that $\lambda \geq \frac{2}{\sqrt{6}} c$. If $\mu>\frac{1}{2 \sqrt{6}}$ then we have $u^{(1)} \sim e^{\left(\frac{5}{2 \sqrt{6}} c t-(\lambda+c \mu) t\right)}$ as $t \rightarrow \infty$. In order to have a bounded solution $u^{(1)}$ as $t \rightarrow \infty$ we require that $\lambda+c \mu>\frac{5}{2 \sqrt{6}} c$.

For fixed $t$, we have $u^{(1)} \sim O(1) e^{-\frac{5}{2 \sqrt{6}} x} e^{-\left(\frac{2}{\sqrt{6}}+\mu\right) x}$ as $x \rightarrow \infty$, which means that $u^{(1)} \rightarrow 0$ as $x \rightarrow \infty$.

For $x<0$ the solution $(4.43)$ can be written as

$$
u^{(1)}(x, t) \sim h(x-c t) \exp \left(-\frac{5}{2 \sqrt{6}} x\right) \exp \left(\frac{5}{2 \sqrt{6}} c t-(\lambda-c \mu) t\right) .
$$

For the cases where $h_{1}$ and $h_{2}$ are exponential functions, we have

$$
h \sim A e^{\frac{7}{2 \sqrt{6}} \xi}+B e^{\frac{-7}{2 \sqrt{6}} \xi}+e^{\left(\frac{9}{2 \sqrt{6}}+\mu\right) \xi}, \quad \xi \rightarrow \infty
$$

which gives

$$
\begin{gathered}
u^{(1)}(x, t) \sim\left(A e^{\frac{7}{2 \sqrt{6}} \xi}+B e^{\frac{-7}{2 \sqrt{6}} \xi}+e^{\left(\frac{9}{2 \sqrt{6}}+\mu\right) \xi}\right) e^{\left(-\frac{5}{2 \sqrt{6}} x\right)} e^{\left(\frac{5}{2 \sqrt{6}} c t-(\lambda-c \mu) t\right)} \\
\sim\left(A e^{\frac{1}{\sqrt{6}} x}+B e^{\frac{-6}{\sqrt{6}} x}+e^{\left(\frac{2}{2 \sqrt{6}}+\mu\right) x}\right)\left(A e^{-\frac{1}{\sqrt{6}} c t-(\lambda-c \mu) t}+B e^{\frac{6}{\sqrt{6}} c t-(\lambda-c \mu) t}+e^{-\left(\frac{2}{\sqrt{6}}+\lambda\right) t}\right), \quad(x-c t) \rightarrow \infty
\end{gathered}
$$

For fixed $x$, as $t \rightarrow \infty$, we have

$$
u^{(1)} \sim A e^{-\frac{1}{\sqrt{6}} c t-(\lambda-c \mu) t}+B e^{\frac{6}{\sqrt{6}} c t-(\lambda-c \mu) t}+e^{-\left(\frac{2}{\sqrt{6}}+\lambda\right) t} .
$$

The dominant term in $u^{(1)}$ is $B e^{\frac{6}{2 \sqrt{6}} c t-(\lambda-c \mu) t}$. In order to have a bounded solution $u^{(1)}$, as $t \rightarrow \infty$ we require that $\lambda-c \mu \geq \frac{6}{\sqrt{6}} c$. If $\lambda-c \mu<\frac{6}{\sqrt{6}} c$, then this term grows without bound as $t \rightarrow \infty$. In order to have a bounded solution, $u^{(1)}$ as $x \rightarrow-\infty$ we must set $B=0$. After that, considering the dominant term, we see 
that $u^{(1)} \sim A e^{-\frac{1}{\sqrt{6}} c t-(\lambda-c \mu) t}$ as $t \rightarrow \infty$. In order to have a bounded solution $u^{(1)}$ as $t \rightarrow \infty$, we require that $\lambda-c \mu \geq \frac{1}{\sqrt{6}} c$.

For fixed $t$, as $x \rightarrow-\infty$, we have

$$
u^{(1)} \sim A e^{\frac{1}{\sqrt{6}} x}+B e^{\frac{-6}{\sqrt{6}} x}+e^{\left(\frac{1}{\sqrt{6}}+\mu\right) x} .
$$

The dominant term in $u^{(1)}$ is $B e^{\frac{-6}{\sqrt{6}} x}$, which means that the solution grows without bound as $x \rightarrow-\infty$. In order to have a bounded solution $u^{(1)}$ as $x \rightarrow-\infty$, we must set $B=0$. For the cases where $h_{1}$ and $h_{2}$ are oscillatory or approximately constant, we have

$$
h \sim O(1)+e^{\left(\frac{9}{2 \sqrt{6}}+\mu\right) \xi}, \quad \xi \rightarrow \infty
$$

which gives

$$
u^{(1)}(x, t) \sim\left(O(1)+e^{\left(\frac{9}{2 \sqrt{6}}+\mu\right) x-c t}\right) e^{\left(-\frac{5}{2 \sqrt{6}} x\right)} e^{\left(\frac{5}{2 \sqrt{6}} c t-(\lambda-c \mu) t\right)} .
$$

For fixed $x$, as $t \rightarrow \infty$, we have

$$
u^{(1)}(x, t) \sim e^{\left(\frac{5}{2 \sqrt{6}} c t-(\lambda-c \mu) t\right)}+e^{-\left(\frac{2}{\sqrt{6}} c+\lambda\right) t} .
$$

In order to have a bounded solution $u^{(1)} \rightarrow 0$ as $t \rightarrow \infty$ we require that $\lambda-c \mu>$ $\frac{5}{2 \sqrt{6}} c$. Alternately, if we set $A=B=0$, them

$$
u^{(1)}(x, t) \sim e^{-\left(\frac{2}{\sqrt{6}} c+\lambda\right) t} \quad t \rightarrow \infty .
$$

which means that $u^{(1)} \rightarrow 0$ as $t \rightarrow \infty$.

For fixed $t$, as $x \rightarrow-\infty$,

$$
u^{(1)}(x, t) \sim(A O(1)+B O(1)) e^{\left(-\frac{5}{2 \sqrt{6}} x\right)}+e^{\left(\frac{2}{\sqrt{6}}+\mu\right) x} .
$$

If $A \neq 0$ and $B \neq 0$, then the solution grows without bound as $x \rightarrow-\infty$. In order to have a bounded solution $u^{(1)}$ as $x \rightarrow-\infty$, we must set $A=B=0$. In that case $u^{(1)} \sim e^{\left(\frac{2}{\sqrt{6}}+\mu\right) x}$ as $x \rightarrow-\infty$.

The conditions for $u^{(1)} \rightarrow 0$ as $t \rightarrow \infty$ and $u^{(1)} \rightarrow 0$ as $x \rightarrow \pm \infty$ are summarize in Table 4.2. If $u^{(1)} \rightarrow 0$ as $\rightarrow \infty$ then $u(x, t)$ converges to the leading order travelling wave solution $U^{(0)}(x-c t)$ as $t \rightarrow \infty$. 


\begin{tabular}{|c|c|c|}
\hline$x>0$ & $t \rightarrow \infty$ & $x \rightarrow \infty$ \\
\hline$h_{1}, h_{2}$ exponential & $\frac{1}{24}>\lambda+c \mu>\frac{3}{\sqrt{6}} c$ & $\lambda+c \mu<\frac{1}{24}$ \\
\hline $\begin{array}{l}h_{1}, h_{2} \text { oscillatory or approx- } \\
\text { imately constant }\end{array}$ & $\begin{array}{l}\mu<\frac{1}{2 \sqrt{6}}, \lambda>\frac{1}{24}-\frac{1}{2 \sqrt{6}} c \\
\text { or } \mu>\frac{1}{2 \sqrt{6}}, \lambda+c \mu>\frac{1}{24}\end{array}$ & $\lambda+c \mu>\frac{1}{24}$ \\
\hline$x<0$ & $t \rightarrow \infty$ & $x \rightarrow-\infty$ \\
\hline$h_{1}, h_{2}$ exponential & $\begin{array}{l}\frac{1}{24} \leq \lambda-c \mu \leq \frac{49}{24} \text { or } \\
B=0, \frac{1}{24} \leq \lambda-c \mu<\frac{49}{24}\end{array}$ & $B=0, \frac{1}{24} \leq \lambda-c \mu<\frac{49}{24}$ \\
\hline $\begin{array}{l}h_{1}, h_{2} \text { oscillatory or approx- } \\
\text { imately constant }\end{array}$ & $\begin{array}{l}\lambda-\mu>\frac{5}{2 \sqrt{6}} c \text { or } \\
A=B=0, \lambda-c \mu \geq \frac{49}{24}\end{array}$ & $\begin{array}{l}A=B=0 \\
\lambda-c \mu \geq \frac{49}{24}\end{array}$ \\
\hline
\end{tabular}

Table 4.2: Summary of the conditions that make $u^{(1)} \rightarrow 0$ as $t \rightarrow \infty$ and $x \rightarrow \pm \infty$ for the solution (4.43)

\subsection{Perturbation to the Velocity}

In this section we perturb the travelling wave solution of the Fisher equation (1.4) by adding a perturbation that depends on $x$ and $t$ to the constant velocity $\bar{c}$ of the background medium. We write $\bar{c}(x, t)=\bar{c}^{(0)}+\varepsilon \bar{c}^{(1)}(x, t)$ so that (1.4) becomes

$$
\frac{\partial u}{\partial t}+\left(\bar{c}^{(0)}+\varepsilon \bar{c}^{(1)}(x, t)\right) \frac{\partial u}{\partial x}=\frac{\partial^{2} u}{\partial x^{2}}+u(1-u) .
$$

Asymptotic expressions for the perturbed solution can be found by making use of the solutions found in section 4.3. As mentioned in chapter 1, this problem where the speed of the background medium is perturbed by a spatially and temporally localized perturbation arises in certain physical situations. For example, in atmospheric fluid flows, wave interactions can perturb the background wind which then affects the advection of chemical species in the atmosphere.

In order to derive an asymptotic solution for this configuration we apply the 
initial condition

$$
u(x, 0)=\frac{e^{\frac{2}{\sqrt{6}} x}}{\left(e^{\frac{1}{\sqrt{6}} x}+b\right)^{2}}=f^{(0)}(x) .
$$

We write the solution of (4.46) as

$$
u(x, t)=u^{(0)}(x, t)+\varepsilon u^{(1)}(x, t)+O\left(\varepsilon^{2}\right) .
$$

Substituting this into (4.46) gives at $O\left(\varepsilon^{0}\right)$

$$
\begin{aligned}
\frac{\partial u^{(0)}}{\partial t}+ & \bar{c} \frac{\partial u^{(0)}}{\partial x}+\varepsilon\left(\frac{\partial u^{(1)}}{\partial t}+\bar{c}^{(0)} \frac{\partial u^{(1)}}{\partial x}-\bar{c}^{(1)}(x, t) \frac{\partial u^{(0)}}{\partial x}\right) \\
& =\frac{\partial^{2} u^{(0)}}{\partial x^{2}}+u^{(0)}\left(1-u^{(0)}\right)+\varepsilon\left(\frac{\partial^{2} u^{(1)}}{\partial x^{2}}-2 u^{(0)} u^{(1)}+u^{(1)}\right)+O\left(\varepsilon^{2}\right) .
\end{aligned}
$$

This shows that $u^{(0)}$ satisfies the Fisher equation (1.4) with the initial condition (4.47). Thus,

$$
u^{(0)}(x, t)=U^{(0)}(\xi)=f^{(0)}(\xi)=f^{(0)}(x-c t)
$$

At $O(\varepsilon)$ we obtain

$$
\frac{\partial u^{(1)}}{\partial t}+\bar{c} \frac{\partial u^{(1)}}{\partial x}+\bar{c}^{(1)}(x, t) \frac{\partial u^{(0)}}{\partial x}=\frac{\partial^{2} u^{(1)}}{\partial x^{2}}+\left(1-2 u^{(0)}\right) u^{(1)} .
$$

The analysis of section 4.3 suggests that there is a solution of (4.50) of the form $u^{(1)}(x, t)=\alpha(\xi) \beta(x, t)$, where $\xi=x-c t$. Substituting this into (4.50) gives

$$
\begin{aligned}
\alpha_{\xi \xi}+(c-\bar{c}) \alpha_{\xi}+\left(1-2 f^{(0)}\right) & \alpha \\
& =\left(\frac{\beta_{t}+\bar{c} \beta_{x}-\beta_{x x}}{\beta}\right) \alpha+\frac{\bar{c}^{(1)}}{\beta} f_{\xi}^{(0)}-\frac{2 \beta_{x}}{\beta} \alpha_{\xi} .
\end{aligned}
$$

Since the left hand side depends on $\xi$ only, the right hand side should also depend on $\xi$ only and hence we must choose $\beta(x, t)=\bar{c}^{(1)}(x, t)$. We choose $\bar{c}^{(1)}(x, t)$ to be a product of exponential functions $e^{-\lambda t}$ and $e^{-\mu x}$ In order for the solution to satisfy the initial condition (4.47), we must have $\bar{c}^{(1)}(x, 0)=0$. An appropriate choice of $\bar{c}^{(1)}$ is thus $\bar{c}^{(1)}(x, t)=e^{-\lambda\left|t-t_{1}\right|} e^{-\mu|x|}$, where $t_{1}$ is a positive constant.

For $t>t_{1}$ and $x>0$, we obtain

$$
\alpha_{\xi \xi}+(c-\bar{c}-2 \mu) \alpha_{\xi}+\left(1+\lambda+\bar{c} \mu+\mu^{2}-2 f^{(0)}\right) \alpha=f_{\xi}^{(0)} .
$$


To eliminate the first derivative we make the change of variable

$$
\alpha(\xi)=h(\xi) e^{\frac{-(c-\bar{c}-2 \mu)}{2} \xi} .
$$

Substituting this into (4.52) gives

$$
h_{\xi \xi}-Q(\xi) h=e^{\frac{(c-\bar{c}-2 \mu)}{2} \xi} f_{\xi}^{(0)},
$$

where $Q(\xi)=2 f^{(0)}(\xi)+\frac{(c-\bar{c}-2 \mu)^{2}}{4}-\mu^{2}-\lambda-\bar{c} \mu-1$. Simplifying this expression and setting $c-\bar{c}=\frac{5}{\sqrt{6}}$ gives

$$
Q(\xi)=2 f^{(0)}(\xi)+\frac{1}{24}-\sigma,
$$

where $\sigma=(\lambda+c \mu)$. For $t>t_{1}$ and $x<0$, we obtain the same expression for $Q$ but with $\sigma=\lambda-c \mu$. For $t<t_{1}$ and $x>0$, we obtain the same expression for $Q$ but with $\sigma=-\lambda+c \mu$. For $t<t_{1}$ and $x<0$, we obtain the same expression for $Q$ but with $\sigma=-\lambda-c \mu$.

The solution of the nonhomogeneous equation (4.54) is the sum of a linear combination of solutions of the corresponding homogeneous equation and a particular solution of the nonhomogeneous equation. Asymptotic approximations for the solutions $h_{1}(\xi)$ and $h_{2}(\xi)$ of the homogeneous equation are given by the expressions in section 4.3. For values of $\xi$ for which $Q(\xi)>0$, two linearly independent solutions are given in (4.40). For values of $\xi$ for which $Q(\xi)<0$, two linearly independent homogeneous solutions are given in (4.41). As in section 4.3.2, the form of the solutions depends on the value of $\sigma$. The different possibilities are summarized in Table 4.1.

Once the solutions of the homogeneous equation are determined, a particular solution of the nonhomogeneous equation can be obtained using the method of variation of parameters. The particular solution is

$$
h_{p}(\xi)=h_{2} \int \frac{h_{1} F(\xi)}{W\left(h_{1}, h_{2}\right)} d \xi-h_{1} \int \frac{h_{2} F(\xi)}{W\left(h_{1}, h_{2}\right)} d \xi,
$$


where $F$ is take the form $F(\xi)=e^{\frac{(c-\bar{c}-2 \mu)}{2} \xi} f_{\xi}^{(0)}$ if $x>0$ and $F(\xi)=e^{\frac{(c-\bar{c}+2 \mu)}{2} \xi} f_{\xi}^{(0)}$ if $x<0$, and $W\left(h_{1}, h_{2}\right)$ is the Wronskian which is defined as $W\left(h_{1}, h_{2}\right)(\xi)=$ $h_{1} h_{2 \xi}-h_{1 \xi} h_{2}$. For the values of $\xi$ for which $Q(\xi)>0, W$ is given by

$$
W\left(h_{1}, h_{2}\right)=-2+\frac{Q_{\xi}}{2 Q^{\frac{3}{2}}}
$$

and for the values of $\xi$ for which $Q(\xi)<0$,

$$
W\left(h_{1}, h_{2}\right)=1+\frac{Q_{\xi} \sin \left(\int_{a}^{\xi} \sqrt{-Q} d \bar{\xi}\right) \cos \left(\int_{a}^{\xi} \sqrt{-Q} d \bar{\xi}\right)}{2 Q^{\frac{3}{2}}} .
$$

The solution of the nonhomogeneous equation (4.54) is then

$$
h(\xi)=A h_{1}(\xi)+B h_{2}(\xi)+h_{p}(\xi)
$$

where $A$ and $B$ are constants and the solution of (4.50) is

$$
u^{(1)}(x, t)=h(x-c t) \exp \left(\frac{-(c-\bar{c}-2(\operatorname{sgn} x) \mu)}{2}(x-c t)-\mu|x|-\lambda\left|t-t_{1}\right|\right) .
$$

For the values of $\sigma$ for which $h_{1}$ and $h_{2}$ are exponential functions, as $\xi \rightarrow \infty$, $f^{(0)}(\xi) \sim e^{-\frac{2}{\sqrt{6}} \xi}, f_{\xi}^{(0)}(\xi) \sim e^{-\frac{2}{\sqrt{6}} \xi}$, so $F(\xi) \sim e^{\left(\frac{5}{2 \sqrt{6}}+\mu\right) \xi} e^{-\frac{2}{\sqrt{6}} \xi} \sim e^{\left(\frac{1}{2 \sqrt{6}}-\mu\right) \xi}$ and $h_{1}(\xi) \sim e^{\frac{1}{2 \sqrt{6}} \xi}, h_{2}(\xi) \sim e^{-\frac{1}{2 \sqrt{6}} \xi}$, according to (4.28). Thus, $W \sim-2$ and $h_{p} \sim$ $e^{\left(\frac{1}{2 \sqrt{6}}-\mu\right) \xi}$. For the values of $\sigma$ for which $h_{1}$ and $h_{2}$ are oscillatory or approximately constant so $h_{1,2} \sim O(1)$ and $F(\xi) \sim e^{\left(\frac{5}{2 \sqrt{6}}+\mu\right) \xi} e^{-\frac{2}{\sqrt{6}} \xi} \sim e^{\left(\frac{1}{2 \sqrt{6}}-\mu\right) \xi}$, thus $h_{p} \sim$ $e^{\left(\frac{1}{2 \sqrt{6}}-\mu\right) \xi}$ as $\xi \rightarrow \infty$.

For the values of $\sigma$ which $h_{1}$ and $h_{2}$ are exponential functions, as $\xi \rightarrow-\infty$, $f^{(0)}(\xi) \sim 1-e^{\frac{2}{\sqrt{6}} \xi}$, so $F(\xi) \sim e^{\left(\frac{5}{2 \sqrt{6}}+\mu\right) \xi} e^{\frac{2}{\sqrt{6}} \xi} \sim e^{\left(\frac{9}{2 \sqrt{6}}+\mu\right) \xi}$ and $h_{1}(\xi) \sim e^{\frac{7}{2 \sqrt{6}} \xi}$ and $h_{2}(\xi) \sim e^{-\frac{7}{2 \sqrt{6}} \xi}$, according to $(4.26)$. Thus, $W \sim-2$ and $h_{p} \sim e^{\left(\frac{9}{2 \sqrt{6}}+\mu\right) \xi}$. For the values of $\sigma$ for which $h_{1}$ and $h_{2}$ are oscillatory or approximately constant, $h_{1,2} \sim O(1)$ and $F(\xi) \sim e^{\left(\frac{5}{2 \sqrt{6}}+\mu\right) \xi} e^{\frac{2}{\sqrt{6}} \xi} \sim e^{\left(\frac{9}{2 \sqrt{6}}+\mu\right) \xi}, h_{p} \sim e^{\left(\frac{9}{2 \sqrt{6}}+\mu\right) \xi}$ as $\xi \rightarrow-\infty$.

For $x>0$ and $t>t_{1}$, the solution (4.58) can be written as

$$
u^{(1)}(x, t)=h(x-c t) \exp \left(-\frac{5}{2 \sqrt{6}} x\right) \exp \left(\frac{5}{2 \sqrt{6}} c t-(\lambda+c \mu) t+\lambda t_{1}\right)
$$


This is the same form as the solution (4.44) in Section 4.3.2 and we obtain the same conditions on the constants $\sigma$ and $\mu$ to have $u^{(1)} \rightarrow 0$ as $t \rightarrow \infty$ and as $x \rightarrow \infty$. These conditions are summarized in Table 4.3.

For $x<0$ and $t>t_{1}$, the solution (4.58) can be written as

$$
u^{(1)}(x, t) \sim h(x-c t) \exp \left(-\frac{5}{2 \sqrt{6}} x\right) \exp \left(\frac{5}{2 \sqrt{6}} c t-(\lambda-c \mu) t+\lambda t_{1}\right) .
$$

This is the same form as the solution (4.60) in Section 4.3.2 and we obtain the same conditions on the constants $\sigma$ and $\mu$ to have $u^{(1)} \rightarrow 0$ as $t \rightarrow \infty$ and as $x \rightarrow-\infty$. These conditions are summarized in Table 4.3.

For $x>0$ and $t<t_{1}$, the solution (4.58) can be written as

$$
u^{(1)}(x, t) \sim h(x-c t) \exp \left(-\frac{5}{2 \sqrt{6}} x\right) \exp \left(\frac{5}{2 \sqrt{6}} c t+(\lambda-c \mu) t-\lambda t_{1}\right) .
$$

In this case for fixed $t$ as $x \rightarrow \infty$, we have the same equation (4.59).

For the cases where $h_{1}$ and $h_{2}$ are exponential functions, we have

$$
h \sim A e^{\frac{1}{2 \sqrt{6}} \xi}+B e^{\frac{-1}{2 \sqrt{6}} \xi}+e^{\left(\frac{1}{2 \sqrt{6}}-\mu\right) \xi}, \quad \xi \rightarrow \infty
$$

which gives

$$
u^{(1)} \sim\left(A e^{\frac{(x-c t)}{2 \sqrt{6}}}+B e^{\frac{-(x-c t)}{2 \sqrt{6}}}+e^{\left(\frac{1}{2 \sqrt{6}}-\mu\right)(x-c t)}\right) e^{-\frac{5}{2 \sqrt{6}} x} e^{\left(\frac{5}{2 \sqrt{6}} c t+(\lambda-c \mu) t\right)} .
$$

For fixed $t$, as $x \rightarrow \infty$, we have

$$
\begin{gathered}
u^{(1)} \sim\left(A e^{\frac{1}{2 \sqrt{6}} x}+B e^{\frac{-1}{2 \sqrt{6}} x}+e^{\left(\frac{1}{2 \sqrt{6}}-\mu\right) x}\right) e^{\left(-\frac{5}{2 \sqrt{6}}\right) x}, \\
\sim\left(A e^{\frac{-2}{\sqrt{6}} x}+B e^{\frac{-3}{\sqrt{6}} x}+e^{-\left(\frac{2}{2 \sqrt{6}}+\mu\right) x}\right) .
\end{gathered}
$$

Considering the dominant term, we can see that $u^{(1)} \sim e^{-\frac{3}{2 \sqrt{6}} x}$ as $x \rightarrow \infty$, which means that $u^{(1)} \rightarrow 0$ as $x \rightarrow \infty$.

For the cases where $h_{1}$ and $h_{2}$ are oscillatory or approximately constant we have

$$
h \sim O(1)+e^{\left(\frac{1}{2 \sqrt{6}}-\mu\right) \xi}, h \text { space } 5 m m \xi \rightarrow \infty,
$$


So

$$
\begin{gathered}
u^{(1)} \sim\left(O(1)+e^{\left(\frac{1}{2 \sqrt{6}}-\mu\right)(x-c t)}\right) e^{-\frac{5}{2 \sqrt{6}} x} e^{\left(\frac{5}{2 \sqrt{6}} c t-(\lambda+c \mu) t\right)} \\
u^{(1)} \sim e^{-\frac{5}{2 \sqrt{6}} x} e^{\left(\frac{5}{2 \sqrt{6}} c t-(\lambda+c \mu) t\right)}+e^{\left(\frac{-2}{\sqrt{6}}-\mu\right) x} e^{\left(\frac{2}{\sqrt{6}} c-\lambda\right) t}
\end{gathered}
$$

Considering the dominant term, we have $u^{(1)} \sim(A O(1)+B O(1)) e^{-\frac{5}{2 \sqrt{6}} x}+e^{-\left(\frac{2}{\sqrt{6}}+\mu\right) x}$ as $x \rightarrow \infty$, which means that $u^{(1)} \rightarrow 0$ as $x \rightarrow \infty$.

For $x<0$ and $t<t_{1}$, the solution (4.58) can be written as

$$
u^{(1)}(x, t) \sim h(x-c t) \exp \left(-\frac{5}{2 \sqrt{6}} x\right) \exp \left(\frac{5}{2 \sqrt{6}} c t+(\lambda+c \mu) t-\lambda t_{1}\right) .
$$

In this case for fixed $t$ as $x \rightarrow-\infty$, we have the same equation (4.60).

For the cases where $h_{1}$ and $h_{2}$ are exponential functions, we have

$$
h \sim A e^{\frac{7}{2 \sqrt{6}} \xi}+B e^{\frac{-7}{2 \sqrt{6}} \xi}+e^{\left(\frac{9}{2 \sqrt{6}}+\mu\right) \xi}, \quad \xi \rightarrow-\infty
$$

which gives

$$
u^{(1)}(x, t) \sim\left(A e^{\frac{7}{2 \sqrt{6}} \xi}+B e^{\frac{-7}{2 \sqrt{6}} \xi}+e^{\left(\frac{9}{2 \sqrt{6}}+\mu\right) \xi}\right) e^{\left(-\frac{5}{2 \sqrt{6}} x\right)} e^{\left(\frac{5}{2 \sqrt{6}} c t+(\lambda+c \mu) t\right)}
$$

For fixed $t$, as $x \rightarrow-\infty$, we have

$$
u^{(1)} \sim\left(A e^{\frac{1}{\sqrt{6}} x}+B e^{\frac{-6}{\sqrt{6}} x}+e^{\left(\frac{2}{2 \sqrt{6}}+\mu\right) x}\right) .
$$

The dominant term in $u^{(1)}$ is $B e^{\frac{-6}{\sqrt{6}} x}$, which means that the solution grows without bound as $x \rightarrow-\infty$. In order to have a bounded solution $u^{(1)} \rightarrow 0$ as $x \rightarrow-\infty$, we must set $B=0$.

For the cases where $h_{1}$ and $h_{2}$ are oscillatory or approximately constant, we have

$$
h \sim A O(1)+B O(1)+e^{\left(\frac{9}{2 \sqrt{6}}+\mu\right) \xi},
$$

which gives

$$
u^{(1)}(x, t) \sim\left(A O(1)+B O(1)+e^{\left(\frac{9}{2 \sqrt{6}}+\mu\right) \xi}\right) e^{\left(-\frac{5}{2 \sqrt{6}} x\right)} e^{\left(\frac{5}{2 \sqrt{6}} c t+(\lambda+c \mu) t\right)}
$$


For fixed $t$, as $x \rightarrow-\infty$,

$$
u^{(1)}(x, t) \sim e^{\left(-\frac{5}{2 \sqrt{6}} x\right)}+e^{\left(\frac{2}{\sqrt{6}}+\mu\right) x} .
$$

If $A \neq 0$ and $B \neq 0$. then the solution grows without bound as $x \rightarrow-\infty$. In order to have a bounded solution we must set $A=B=0$. In that case $u^{(1)} \sim e^{\left(\frac{2}{\sqrt{6}}+\mu\right) x}$ as $x \rightarrow-\infty$.

The conditions for $u^{(1)} \rightarrow 0$ as $t \rightarrow \infty$ and $u^{(1)} \rightarrow 0$ as $x \rightarrow \pm \infty$ for $t<t_{1}$ are summarize in Table 4.2. These are the same condition as in Table 4.2. for the configuration where the initial condition is perturbed. If $u^{(1)} \rightarrow 0$ as $t \rightarrow \infty$ then $u(x, t)$ converges to the leading order travelling wave solution $U^{(0)}(x-c t)$ as $t \rightarrow \infty$. The conditions for $u^{(1)} \rightarrow 0$ as $x \rightarrow \pm \infty$ for $t<t_{1}$ also are given in Table 4.2 . 


\begin{tabular}{|c|c|c|}
\hline$x>0$ and $t>t_{1}$ & $t \rightarrow \infty$ & $x \rightarrow \infty$ \\
\hline$h_{1}, h_{2}$ exponential & $\frac{1}{24}>\lambda+c \mu>\frac{3}{\sqrt{6}} c$ & $\lambda+c \mu<\frac{1}{24}$ \\
\hline $\begin{array}{l}h_{1}, h_{2} \text { oscillatory or approx- } \\
\text { imately constant }\end{array}$ & $\begin{array}{l}\mu<\frac{1}{2 \sqrt{6}}, \lambda>\frac{1}{24}-\frac{1}{2 \sqrt{6}} c \\
\text { or } \mu>\frac{1}{2 \sqrt{6}}, \lambda+c \mu>\frac{1}{24}\end{array}$ & $\lambda+c \mu>\frac{1}{24}$ \\
\hline$x<0$ and $t>t_{1}$ & $t \rightarrow \infty$ & $x \rightarrow-\infty$ \\
\hline$h_{1}, h_{2}$ exponential & $\begin{array}{l}\frac{1}{24} \leq \lambda-c \mu \leq \frac{49}{24} \text { or } \\
B=0, \frac{1}{24} \leq \lambda-c \mu<\frac{49}{24}\end{array}$ & $B=0, \frac{1}{24} \leq \lambda-c \mu<\frac{49}{24}$ \\
\hline $\begin{array}{l}h_{1}, h_{2} \text { oscillatory or approx- } \\
\text { imately constant }\end{array}$ & $\begin{array}{l}\lambda-\mu>\frac{5}{2 \sqrt{6}} c \text { or } \\
A=B=0, \lambda-\mu c \geq \frac{49}{24}\end{array}$ & $\begin{array}{l}A=B=0 \\
\lambda-\mu c \geq \frac{49}{24}\end{array}$ \\
\hline$x>0$ and $t<t_{1}$ & & $x \rightarrow \infty$ \\
\hline$h_{1}, h_{2}$ exponential & & $-\lambda+c \mu<\frac{1}{24}$ \\
\hline $\begin{array}{l}h_{1}, h_{2} \text { oscillatory or approx- } \\
\text { imately constant }\end{array}$ & & $-\lambda+c \mu>\frac{1}{24}$ \\
\hline$x<0$ and $t<t_{1}$ & & $x \rightarrow-\infty$ \\
\hline$h_{1}, h_{2}$ exponential & & $B=0, \frac{1}{24} \leq-\lambda-c \mu<\frac{49}{24}$ \\
\hline $\begin{array}{l}h_{1}, h_{2} \text { oscillatory or approx- } \\
\text { imately constant }\end{array}$ & & $\begin{array}{l}A=B=0 \\
-\lambda-\mu c \geq \frac{49}{24}\end{array}$ \\
\hline
\end{tabular}

Table 4.3: Summary of the conditions that make $u^{(1)} \rightarrow 0$ as $t \rightarrow \infty$ and $x \rightarrow \pm \infty$ for solution (4.58) 


\section{Chapter 5}

\section{Conclusions}

In this thesis we examined some nonlinear advection-diffusion-reaction equations of the Fisher type. First we discussed the stability properties of equations of the form (1.1)-(1.2) by writing each nonlinear equation as a system of two ODEs and then analyzing the stability of their equilibrium solutions and plotting their trajectories in phase portraits.

For equation (1.1) with the nonlinear term (1.2) $F(u)=u\left(\gamma-\beta u^{p}\right)$ there are three critical points or equilibrium solutions if $p$ is even and two critical points or equilibrium solutions if $p$ is not even. There is a zero critical point which is asymptotically stable if $c>0$ and unstable if $c<0$ and there are two saddle points if $p$ is even and one saddle point if $p$ is not even. The phase portraits for the linear systems obtained by linearizing around each of these critical points are shown in Figures 2.1-2.3. The phase portraits for the nonlinear system are shown in Figures 2.4-2.7. For equation (1.1) with the nonlinear term (1.3) $F(u)=u\left(\gamma-\beta u-\alpha u^{2}\right)$, there are three critical points or equilibrium solutions. There is a zero critical point which is asymptotically stable if $c>0$ and unstable if $c<0$ and there are two saddle points.

The rest of the thesis was focussed on the case with equation (1.1) with the nonlinear term $F(u)=u\left(\gamma-\beta u^{p}\right)$. A number of previous studies derived exact 
travelling wave solutions for different variants and special cases of this equation using different methods e.g. Ablowitz and Zeppetella (1979), Abdelkader (1982), Feng (2007) and Yuan et al. (2013).

We used the transformation of Feng (2007) to derive travelling wave solutions which had been obtained other researchers (e.g Yuan et al. (2013)) using other methods. Szozda (2004) had previously used this transformation for the case where $F(u)=u\left(1-u^{p}\right)$, with $p=1,2$. Here we considered $F(u)=u\left(\gamma-\beta u^{p}\right)$ with general real positive $\gamma, \beta$ and $p$.

We then considered the situation where these travelling wave solutions are perturbed. The stability of perturbed travelling wave solutions was examined by Murray (1989). Here we considered some special situations that can lead to perturbations. First we perturbed the initial condition and then derived approximation expressions (4.40) and (4.41) for the perturbations using the WKB approximation.

The goal of the thesis was to investigate the case where the constant speed $\bar{c}$ of propagation of the background medium is perturbed by a small amplitude spatially and temporally localized perturbation. This configuration is relevant in certain situations where atmospheric waves affect the propagation of chemical species in the atmosphere. We added a perturbation by writing $\bar{c}=\bar{c}^{(0)}+\varepsilon \bar{c}^{(1)}(x, t)$, where $\bar{c}^{(1)}(x, t)=e^{-\mu|x|} e^{-\lambda\left|t-t_{1}\right|}$ in (1.4) to obtain equation (4.46). We considered the initial-value problem where (4.46) is subject to the initial condition $u(x, t)=f^{(0)}(x)$. This gave a solution $u(x, t) \sim \bar{u}^{(0)}(x, t)+\varepsilon \bar{u}^{(1)}(x, t)$, where $u^{(1)}$ was written as $u^{(1)}(x, t)=h(\xi) e^{\frac{-(c-\bar{c}-2 \mu)}{2}} e^{-\mu|x|} e^{-\lambda\left|t-t_{1}\right|}$ with $\xi=x-c t$. We used the WKB method to obtain approximation expressions for the function $h(\xi)$. We found that the form of the solution depends on the value of the constant $\sigma=(\lambda+c \mu)$ as summarized in Table 4.1.

Thus the form of the perturbation, whether it is oscillatory or exponentially decaying, depends on $\lambda$ and $\mu$ which define the width of the perturbation in time 
and space. For the values of $\lambda$ and $\mu$ which are given in Table 4.2 and 4.3 satisfies $u^{(1)} \rightarrow 0$ as $x \rightarrow \pm \infty$ and $u^{(1)} \rightarrow 0$ as $t \rightarrow \infty$. In that case, $u \rightarrow U^{(0)}$. We found that in all cases the factor $e^{-\mu|x|}$ does not appear explicitly in the solution for $u^{(1)}$ (4.59)-(4.62). However, the parameter $\mu$ determines the rate of exponential decay of the perturbation solution with time.

In order to be able to derive asymptotic solutions, we only considered the special case where $\bar{c}^{(0)}$ is constant and $\bar{c}^{(1)}$ takes the form $\bar{c}^{(1)}(x, t)=e^{\frac{-(c-\bar{c}-2 \mu)}{2}} e^{-\mu|x|} e^{-\lambda\left|t-t_{1}\right|}$. Other more general functions could be used for $\bar{c}^{(0)}$ and $\bar{c}^{(1)}$ (with $\bar{c}^{(1)} \rightarrow 0$ as $x \rightarrow \pm \infty)$ and the solution $u^{(1)}$ could be obtained by numerical computations. 


\section{Appendix A}

\section{Definitions and Theorems for the stability of systems of ODEs}

In this appendix some definition and theorems concerning the stability of systems of ODEs are given. The wording of the definitions follows the presentation of Brauer and Nohel (1969) and Strogatz (2014) and the proofs of the theorems are given there.

We consider a first order system of ordinary differential equations of the form

$$
\mathbf{y}^{\prime}=\mathbf{f}(\mathbf{y}, t)
$$

where $\mathbf{y} \subset \Omega \in \Re^{n}$ and $t \in \Re$ and $\mathbf{f}$ is a function of $\mathbf{y}$ and $t$ and the prime donates differentiation of $\mathbf{y}$ with respect to $t$.

The system (2.31) discussed in chapter 2 is an example of an autonomous system.

\section{Definition A.1: Autonomous system}

A system of the form (A.1) is said to autonomous system if $\mathbf{f}$ is function of $\mathbf{y}$ and does not depend explicitly on $t$ i.e.,

$$
\mathbf{y}^{\prime}=\mathbf{f}(\mathbf{y})
$$




\section{Definition A.2: Critical Point (Fixed Point)}

A point $y=y_{0} \in C^{n}$ is a critical point of the system (A.2) if $f\left(y_{0}\right)=0$. A critical point of the system(A.2) corresponds to an equilibrium solution of the system.

Equilibrium solution can be classified as stable, asymptotically stable or unstable. The wording of these definitions follows that of Brauer and Nohel (1969). For the proof of the theorems the following norm |.| is used.

\section{Definition A.3: Definition of the norm}

The length (norm) of a vector $\mathbf{y}=\left(y_{1}, y_{2}, \ldots \ldots+y_{n}\right)$ is defined $|\mathbf{y}|=\left|y_{1}\right|+\left|y_{2}\right|+$ $\ldots .+\left|y_{n}\right|=\sum_{i=1}^{n}\left|y_{i}\right|$

\section{Definition A.4: Stability of an equilibrium solution}

An equilibrium solution $\mathbf{y}_{0}$ of (A.2) is said to be stable if for each $\varepsilon>0$ there is a a number $\delta>0$ such that if $\psi(t)$ is a solution of the linear system (A.2) having $\left\|\psi\left(t_{0}\right)-\mathbf{y}_{0}\right\|<\delta$, then the solution $\psi(t)$ exists for all $t \geq t_{0}$ and $\left\|\psi(t)-\mathbf{y}_{0}\right\| \leq \varepsilon$ for $t \geq t_{0}$.

\section{Definition A.5: Asymptotic stability of an equilibrium solution}

An equilibrium solution $\mathbf{y}_{0}$ of (A.2) is said to be asymptotically stable if it is stable and if there exists a number $\delta_{0}>0$ such that if $\psi(t)$ is any solution of the linear system (A.2) having $\left\|\psi\left(t_{0}\right)-\mathbf{y}_{0}\right\|<\delta_{0}$, then $\lim _{t \rightarrow+\infty} \psi(t)=y_{0}$.

\section{Definition A.6: Instability of an equilibrium Solution}

An equilibrium solution $\mathbf{y}_{0}$ is unstable if it is neither stable or asymptotically stable.

The simplest general system for which stability questions are easily and completely decided is the linear system

$$
\mathbf{y}^{\prime}=A \mathbf{y}
$$

where $A$ is a real constant $n \times n$ matrix.

\section{Theorem A.1 Brauer and Nohel (1969)}

If all the eigenvalues of the matrix $A$ have non-positive real parts and all those eigenvalues with zero real parts are simple, then the solution $\mathbf{y}=\mathbf{0}$ of the system 
(A.3) is stable. If and only if all eigenvalues of $A$ have negative real parts, the zero solution of the system (A.3) is asymptotically stable. In fact, in this case if $\Psi\left(t, t_{0}\right)$ denotes the fundamental matrix of the system which is the identity at $t=t_{0}$, then $\Psi\left(t ; t_{0}\right)=\exp \left(\left(t ; t_{0}\right) A\right)$ and there exist constants $k>0$ and $\sigma>0$ such that

$$
\left|\Psi\left(t ; t_{0}\right)\right| \leq k \exp \left(-\sigma\left(t-t_{0}\right)\right)
$$

If all eigenvalues of $\mathrm{A}$ have negative real parts then $\sigma>0$. If there are simple eigenvalues with zero real part, then $\sigma=0$. If one or more eigenvalue has a positive real part, the zero solution is unstable.

The following two theorems give condition for determining whether the zero equation solution $y=0$ of a nonlinear system

$$
\mathbf{y}^{\prime}=A \mathbf{y}+\mathbf{g}(t, \mathbf{y})
$$

is asymptotically stable or unstable. We use the wording of Brauer and Nohel (1969). These results are combined as one theorem Grobman/Hartman (see for Perko (1991)).

\section{Theorem A.2 Brauer and Nohel (1969)}

Consider a nonlinear system written in the form (A.5) Suppose all eigenvalues of

the coefficient matrix $\mathbf{A}$ have negative real parts $\mathbf{g}(\mathbf{t}, \mathbf{y})$ and $\frac{\partial \mathbf{g}}{\partial y_{i}}(j=1 ; \ldots ; n)$ are continuous in $(t, \mathbf{y})$ for $0 \leq t<\infty,|\mathbf{y}|<k$ where $k>0$ is a constant and $\mathbf{g}$ is small in the sense that

$$
\lim _{|\mathbf{y}| \rightarrow 0} \frac{\mid \mathbf{g}(t, \mathbf{y})) \mid}{|\mathbf{y}|}=0
$$

uniformly with respect to $t$ on $0 \leq t<\infty$. Then the solution $\mathbf{y} \equiv \mathbf{0}$ of the system is asymptotically stable.

\section{Theorem A.3 Brauer and Nohel (1969)}

Consider the system

$$
\mathbf{y}^{\prime}=A \mathbf{y}+\mathbf{g}(\mathbf{y})
$$


and

$$
A=\left(\begin{array}{cc}
-\gamma & 0 \\
0 & \lambda
\end{array}\right)
$$

where $\lambda, \gamma>0$.

Let $\mathbf{g}, \frac{\partial \mathbf{g}}{\partial y_{j}},(j=1,2)$ be continuous for $|\mathbf{y}|<k$ for some constant $k>0(k$ can be small) and let $\mathbf{g}(\mathbf{0})=\mathbf{0}$ and $\lim _{|\mathbf{y}| \rightarrow 0}\left|\frac{\partial \mathbf{g}}{\partial y_{i}}\right|=0(i=1,2)$. If the eigenvalues of $A$ are $\lambda,-\gamma$ with $\lambda, \gamma>0$, then zero solution of (A.7) is unstable.

These theorems do not apply to the case where the zero equilibrium solution is stable but not asymptotically stable. In that case, we can use the Lyapunov method.

Let $V(\mathbf{y})$ be a scalar continuous real valued function defined for some region $\Omega$ containing the origin.

\section{Definition A.7: Positive Definite}

The scalar function $V(\mathbf{y})$ is said to be positive definite on set $\Omega$ if and only if $V(0)=0$ and $V(\mathbf{y})>0, \mathbf{y} \neq 0$ and $\forall \mathbf{y} \in \Omega$.

\section{Definition A.8: Derivative $V^{*}$ of the Scalar Function $V$}

The derivative of $V$ with respect to the system (A.2) is the scaler product

$$
V^{*}(\mathbf{y})=\nabla V(\mathbf{y}) \cdot \mathbf{f}(\mathbf{y})
$$

\section{Theorem A.4 Lyapunov Theorem (Brauer and Nohel, 1969)}

For an autonomous system (A.2) if there exists a scalar function $V(\mathbf{y})$ that is positive define and for which the derivative of $V$ with respect (A.2) is nonpositive $\left(V^{*}(\mathbf{y}) \leq 0\right)$ on some region $\Omega$ containing the origin, then the zero solution of (A.2) is stable. 


\section{Appendix B}

\section{The WKB Approximation}

In chapters 4 and 5 we use the Wentzel-Kramers-Brillouin (WKB) method to obtain approximate expressions for the second term in the asymptotic series solutions for some perturbed travelling wave problems.

The WKB method, also sometimes called the Wentzet-Kramers-Brillouin- Jeffreys (WKBJ) method, is a procedure for finding approximate solutions to homogeneous linear differential equations with variable coefficients that vary slowly with respect to the independent variable. In this appendix we give a derivation of the method and the conditions under which it can be applied. The description follows that given in the texts of Nayfeh (1981) and Bender and Orszag (1999).

Consider differential equation

$$
\varepsilon^{2} y^{\prime \prime}=Q(x) y,
$$

where $\varepsilon$ is a small parameter and $Q$ is a real-valued continuous function of $x$ defined on an interval $I \subset \Re$. We seek for a solution for equation (B.1) in the form

$$
y(x) \sim \exp \left(\frac{1}{\sigma} \sum_{n=0}^{\infty} \sigma^{n} S_{n}(x)\right), \quad \sigma \rightarrow 0 .
$$


Differentiating (B.2) gives

$$
\begin{gathered}
y^{\prime}(x) \sim \frac{1}{\sigma} \sum_{n=0}^{\infty} \sigma^{n} S_{n}^{\prime}(x) \exp \left(\frac{1}{\sigma} \sum_{n=0}^{\infty} \sigma^{n} S_{n}(x)\right), \\
y^{\prime \prime}(x) \sim\left(\frac{1}{\sigma^{2}}\left[\sum_{n=0}^{\infty} \sigma^{n} S_{n}^{\prime}(x)\right]^{2}+\frac{1}{\sigma} \sum_{n=0}^{\infty} \sigma^{n} S_{n}^{\prime \prime}(x)\right) \exp \left(\frac{1}{\sigma} \sum_{n=0}^{\infty} \sigma^{n} S_{n}(x)\right),
\end{gathered}
$$

Substituting $y$ and $y^{\prime \prime}$ into equation (B.1) gives

$$
\frac{\varepsilon^{2}}{\sigma^{2}}\left(\sum_{n=0}^{\infty} \sigma^{n} S_{n}^{\prime}(x)\right)^{2}+\frac{\varepsilon}{\sigma} \sum_{n=0}^{\infty} \sigma^{n} S_{n}^{\prime \prime}(x)=Q(x) .
$$

Choosing $\sigma=\varepsilon$ gives

$$
\begin{gathered}
O(1): S_{0}^{\prime 2}=Q(x) \\
O(\varepsilon): S_{0}^{\prime \prime}+2 S_{0}^{\prime} S_{1}^{\prime}=0 \\
O\left(\varepsilon^{n}\right): S_{n-1}^{\prime \prime}+2 S_{0}^{\prime} S_{n}^{\prime}+\sum_{i=1}^{n-1} S_{i}^{\prime} S_{n-1}^{\prime}=0
\end{gathered}
$$

The solution of (B.6) is

$$
S_{0}^{\prime}= \begin{cases} \pm \sqrt{Q(x)} & \text { if } Q(x)>0 \\ \pm i \sqrt{Q(x)} & \text { if } Q(x)<0\end{cases}
$$

This gives

$$
S_{0}= \begin{cases} \pm \int_{a}^{x} \sqrt{Q(\bar{x})} d \bar{x} & \text { if } Q(\bar{x})>0 \\ \pm i \int_{a}^{x} \sqrt{Q(\bar{x})} d \bar{x} & \text { if } Q(\bar{x})<0\end{cases}
$$

From (B.7) we can write $S_{1}$ in terms of $S_{0}$ as

$$
S_{1}=-\frac{1}{2} \ln S_{0}^{\prime}=-\ln \sqrt{S_{0}^{\prime}}
$$

Substituting for $S_{0}$ and $S_{1}$ in (B.2) gives

$$
y(x) \sim \exp \left(\frac{1}{\varepsilon} S_{0}(x)+S_{1}(x)+O(\varepsilon)\right), \quad \varepsilon \rightarrow 0
$$


If $Q(x)>0$ for all $x$ in the interval under consideration then the asymptotic solution in the interval is a linear combination of functions involving exponentials

$$
y \sim \frac{c_{1} \exp \left[\frac{1}{\varepsilon} \int_{a}^{x} \sqrt{Q(x)} d \bar{x}\right]+c_{2} \exp \left[-\frac{1}{\varepsilon} \int_{a}^{x} \sqrt{Q(x)} d \bar{x}\right]}{\sqrt[4]{Q(x)}}
$$

where $c_{1}$ and $c_{2}$ are arbitrary constants and $a$ is an arbitrary fixed value of $x$. If $Q(x)<0$ for all $x$ in the interval under consideration, then the asymptotic solution in the interval is a linear combination of functions involving complex exponentials which can be written in terms of cosine and sine as

$$
y \sim \frac{c_{1} \cos \left[\frac{1}{\varepsilon} \int_{a}^{x} \sqrt{-Q(x)} d \bar{x}\right]+c_{2} \sin \left[\frac{1}{\varepsilon} \int_{a}^{x} \sqrt{-Q(x)} d \bar{x}\right]}{\sqrt[4]{-Q(x)}}
$$

where $c_{1}$ and $c_{2}$ are arbitrary constants and $a$ is an arbitrary fixed value of $x$.

If $Q(x)=0$ at a point $\bar{x} \in I$, then $\bar{x}$ is a turning point for the differential equation. The asymptotic solution is given by (B.13) for $\{x \in I: Q(x)>0\}$ and by (B.14) for $\{x \in I: Q(x)>0\}$.

This method can be applied to an equation of the form

$$
y^{\prime \prime}=Q(\xi) y,
$$

where $Q$ is a slowly-varying function of $\xi,\left|\frac{d Q}{d \xi}\right| \ll 1$. We write $Q$ as $Q(\xi)=q(\tau)$ where $\tau=\sigma \xi$ and we seek a solution $y$ of (B.15) written in terms of the slow variable as

$$
y(\xi)=Y(\tau)
$$

This gives

$$
y^{\prime}=\frac{d y}{d \xi}=\frac{d Y}{d \tau} \frac{d \tau}{d \xi}=\delta \frac{d y}{d \tau}
$$

and

$$
y^{\prime \prime}=\delta^{2} \frac{d^{2} Y}{d \tau^{2}}
$$

and (B.15) can be written as

$$
\delta^{2} \frac{d^{2} Y}{d \tau}=q(\tau) Y
$$


According to (B.13) and (B.14), the function $Y$ can be written as

$$
Y(\tau) \sim \frac{c_{1} \exp \left[\frac{1}{\delta} \int_{a}^{\tau} \sqrt{q(\tau)} d \bar{\tau}\right]+c_{2} \exp \left[-\frac{1}{\delta} \int_{a}^{\tau} \sqrt{q(\tau)} d \bar{\tau}\right]}{\sqrt[4]{q(\tau)}}, \quad \delta \rightarrow 0
$$

for $\tau$ in in interval where $q(\tau)>0$ and

$$
Y(\tau) \sim \frac{c_{1} \cos \left[\frac{1}{\delta} \int_{a}^{\tau} \sqrt{-q(\tau)} d \bar{\tau}\right]+c_{2} \sin \left[\frac{1}{\delta} \int_{a}^{\tau} \sqrt{-q(\tau)} d \bar{\tau}\right]}{\sqrt[4]{-q(\tau)}}, \quad \delta \rightarrow 0
$$

for $\tau$ in the interval where $q(\tau)<0$ and where $c_{1}$ and $c_{2}$ are arbitrary constants and $a$ is an arbitrary fixed value of $\tau$. We can write (B.19) and (B.20) in terms of $\xi$ as

$$
y(\xi) \sim \frac{c_{1} \exp \left[\int_{a}^{\xi} \sqrt{Q(\xi)} d \bar{\xi}\right]+c_{2} \exp \left[-\int_{a}^{\xi} \sqrt{Q(\xi)} d \bar{\xi}\right]}{\sqrt[4]{Q(\xi)}}, \quad|\xi| \rightarrow 0
$$

for $Q(\xi)>0$ and

$$
y(\xi) \sim \frac{c_{1} \cos \left[\int_{a}^{\xi} \sqrt{-Q(\xi)} d \bar{\xi}\right]+c_{2} \sin \left[\int_{a}^{\xi} \sqrt{-Q(\xi)} d \bar{\xi}\right]}{\sqrt[4]{-Q(\xi)}}, \quad|\xi| \rightarrow 0
$$

for $Q(\xi)<0$, where $Q(\xi)=q(\delta \xi),\left|\frac{d Q}{d \xi}\right| \sim \delta \ll 1$ and $c_{1}$ and $c_{2}$ are arbitrary constants and $a$ is an arbitrary fixed value of $\xi$. 


\section{Bibliography}

[1] Abdelkader, M. A., 1982, Travelling wave solutions for a generalized Fisher equation, J. Math. Anal. Appl. 85, 287-290.

[2] Ablowitz, M. J., Zeppetella, A., 1979, Explicit solutions of Fisher's equation for a special wave speed. Bulletin of Mathematical Biology, 41, 835-840.

[3] Abramowitz, M., Stegun, I.A., 1964, Handbook of mathematical functions with formulas, graphs and mathematical tables, Nat. Bur. Stands.

[4] Bender, C. M., Orszag, S. A., 1999, Advanced mathematical methods for scientists and engineers I, Springer Science and Business Media, Chicago.

[5] Bramson, M.D., 1983, Convergence of solutions of the Kolmogorov equation to travelling waves. Mem. Amer. Math. Sco. , 44 (285).

[6] Bramson, M., 1986, Location of the travelling wave for the kolmogorov equation, Probability Theory and Related Fields, 73(4), 481-515.

[7] Brauer, F., Nohel, J. A., 1989, The qualitative theory of ordinary differential equations: An introduction (Dover ed.), Dover, New York.

[8] Canosa, J., 1973, On a nonlinear diffusion equation describing population growth. IBM Journal of Research and Development, 17 (4).

[9] Feng, Z., 2007, Traveling waves to a reaction diffusion equation, Discrete and Continuous Dynamical System Supplement, 38 382-390. 
[10] Fife, P. C., McLeod, J. B., 1975, The approach of solutions of nonlinear diffusion equations to travelling front solutions. Bull. Amer. Math. Soc. ,81(6).

[11] Fife, P. C., McLeod, J. B., 1977, The approach of solutions of nonlinear diffusion equations to travelling front solutions. Archive for Rational Mechanics and Analysis, 65(4).

[12] Fife, P., McLeod, J. B., 1981, A phase plane discussion of convergence to travelling fronts for nonlinear diffusion. Archive for Rational Mechanics and Analysis, 75 (4).

[13] Fisher, R. A., 1937, The wave of advance of advantageous genes. Annals of Human Genetics, 7(4), 355-369.

[14] Kolmogorov, A., Petrovsky I., Piskunov,N., 1937, Etude de lequation de la diffusion avec croissance de la quantite de matiere et son application a un probleme biologique, Moscow Bull. Univ. Math., 1, 125.

[15] Murray, J. D., 1989, Mathematical biology, Springer-Verlag, New York, Berlin.

[16] Nayfeh, A. H., 1981, Introduction to perturbation techniques, Wiley, New York.

[17] Otwinowski, M., Paul, R., Laidlaw, W. G. 1988, Exact travelling wave solutions of a class of nonlinear diffusion equations by reduction to a quadrature, Physics letters A, 128(9), 483-487.

[18] Perko, L. 1991, Differential equations and dynamical systems, SpringerVerlag, New York.

[19] Stockie, J., 2011, The mathematics of atmospheric dispersion modeling. SIAM Review, 53(2), 349-372. 
[20] Strogatz, S. H., 2014,Nonlinear dynamics and chaos: with applications to physics, biology, chemistry, and engineering, Westview press, Chicago.

[21] Tenenbaum, M., Pollard, H., Knovel . 1985, Ordinary differential equations: An elementary textbook for students of mathematics, engineering, and the sciences, Dover Publications, New York.

[22] Uchiyama, K., 1978, The behavior of solutions of some non-linear diffusion equations for large time. J.Math. Kyoto Univ, 18, 453-508.

[23] Szozda, V., 2014, Advection diffusion reaction equations as a model for chemical species in the atmosphere, B.Math Honours Project Report, Carleton University.

[24] Wilhelmsson, H., 1988, Simultaneous diffusion and reaction processes in plasma dynamics. Physical Review A, 38(3), 1482.

[25] Yuan, W., Chen, Q., Qi, J., Li, Y., 2013, The general travelling wave solutions of the Fisher equation with degree three. Advances in Mathematical Physics, 2013 1-5. 